\title{
ANALYSIS OF THE ENU-3 PROTEIN FAMILY IN NERVOUS SYSTEM DEVELOPMENT IN C. ELEGANS
}

by

Roxana Oriana Florica, BScH University of Toronto 2012

\author{
A thesis \\ presented to Ryerson University \\ in partial fulfillment of the \\ requirements for the degree of \\ Master of Science \\ in the Program of \\ Molecular Science
}

Toronto, Ontario, Canada, 2014

(C) Roxana Oriana Florica 2014 
I hereby declare that I am the sole author of this thesis. This is a true copy of the thesis, including any required final revisions, as accepted by my examiners.

I authorize Ryerson University to lend this thesis to other institutions or individuals for the purpose of scholarly research

I further authorize Ryerson University to reproduce this thesis or dissertation by photocopying or by other means, in total or in part, at the request of other institutions or individuals for the purpose of scholarly research.

I understand that my thesis may be made electronically available to the public.

Roxana Oriana Florica 


\author{
$\underline{\text { Abstract }}$ \\ ANALYSIS OF THE ENU-3 PROTEIN FAMILY IN NERVOUS SYSTEM \\ DEVELOPMENT IN C. ELEGANS \\ Master of Molecular Science 2014, \\ Roxana Oriana Florica, \\ Molecular Science Graduate Program, \\ Ryerson University
}

During the development of the nervous system, neurons are guided to their final targets by several well-known guidance cues. In Caenorhabditis elegans the expression of the UNC-6/Netrin guidance cue along the ventral cord attracts axons that express UNC-40, while repulsing axons that express both the UNC-5 and UNC-40 receptors. Lack of both UNC-40 and the novel protein ENU-3 enhanced the ventral guidance defects of the AVM and PVM (Yee et al., 2014). This suggests that ENU-3 functions in an UNC-6 dependent pathway parallel to UNC-40 in controlling migrations towards the ventral nerve cord. Mutations in all proteins of the ENU-3 family also enhance the motor neuron axon outgrowth defects of strains lacking UNC-6 or the UNC-5 receptor, thus they function in a parallel unknown pathway (Yee et al., 2011). Expression analyses in HeLa cells have determined that ENU-3 and one of its paralogs, C38D4.1 localize to the nuclear membrane/ER while another of its paralogs, K01G5.3 is an intracellular membrane-associated protein. 


\section{$\underline{\text { List of Abbreviations }}$}

\begin{tabular}{|c|c|}
\hline BLAST & Basic Local Alignment Search Tool \\
\hline $\mathrm{DCC}$ & Deleted in colorectal cancer \\
\hline DNA & Deoxyribonucleic acid \\
\hline dsRNA & Double-stranded ribonucleic acid \\
\hline Dpy & Dumpy \\
\hline ENU & Enhanced of Uncoordinated \\
\hline GFP & Green fluorescent protein \\
\hline INM & Inner nuclear membrane \\
\hline $\mathrm{mM}$ & Millimolar \\
\hline NGM & Nematode growth media \\
\hline NPC & Nuclear pore complex \\
\hline ONM & Outer nuclear membrane \\
\hline PCR & Polymerase chain reaction \\
\hline Robo & Roundabout \\
\hline RNA & Ribonucleic acid \\
\hline RNAi & Ribonucleic acid interference \\
\hline SNARE & Soluble NSF attachment protein receptor \\
\hline SRP & Signal recognition particle \\
\hline TGF- $\beta$ & Transforming growth factor $\beta$ \\
\hline TM & Transmembrane \\
\hline UNC & Uncoordinated \\
\hline Wnt & Wingless \\
\hline
\end{tabular}




\section{$\underline{\text { Acknowledgements }}$}

I am grateful for the many people that supported me and helped me throughout my career as a graduate student at Ryerson University. I am especially indebted to my supervisor Dr. Marie Killeen for being patient and inspiring me to work harder and persevere through hard times. I am thankful for the interesting project she has given me and the fact that she was always available to discuss experiments. In addition, I am appreciative of my co-supervisor, Dr. Costin Antonescu, for providing great ideas and advice in the collaborative work involving the expression analyses in mammalian cells, as well as providing reagents. I thank the members of my graduate advisory committee, Dr. Costin Antonescu and Dr. Jeffrey Fillingham for their constant support and assistance.

I am thankful for all my friends and colleagues at Ryerson for their help involving experiments in the areas of science with which I was not familiar. I especially thank Camilo Garay, Christian Delos Santos, and Gurgeet Judge, for teaching me IF and Western Blot techniques, as well as making my graduate student experience more enjoyable despite the many hardships along the way. I am also grateful for all the help and support from the amazing past and present undergraduate students in the Killeen lab, especially, Victoria Hipolito, Homai Anvari, Anna Farman, Tiffany Lee and Sushil Khan. I thank members of the Culotti lab at Mt. Sinai Hospital for advising me on microinjection techniques.

I am the most appreciative of my family and Razvan Bobolicu for the daily encouragement and support throughout all steps of my studies. 


\section{Table of Contents}

Author's Declaration...............................................................ii

Abstract....................................................................................iii

List of Abbreviations..............................................................iv

Acknowledgements.......................................................................v

Table of Contents...................................................................vi

List of Tables.........................................................................

List of Figures....................................................................

List of Appendices..............................................................xii

Chapter 1. Introduction...........................................................1

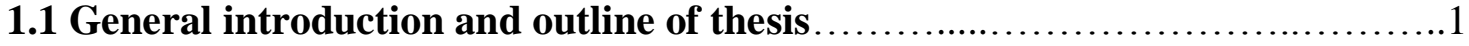

1.2 Caenorhabditis elegans as a model organism .............................

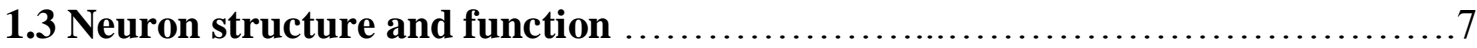

1.4 C.elegans nervous system..............................................

1.5 Growth cones, axon outgrowth and axon guidance........................ 15

1.6 The UNC-6/Netrin signalling pathway ............................... 19

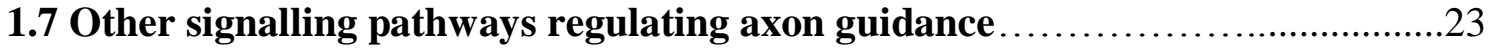

1.8 Discovery of ENU-3 and its role in the nervous system of $C$. elegans............26

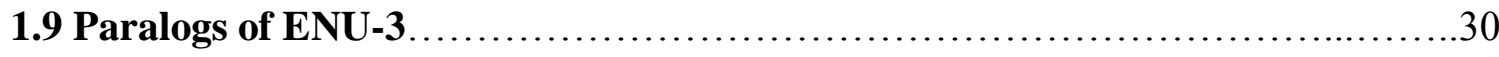

1.10 Nuclear envelope and traffic mechanisms throughout the cell...............32

1.10.1 Structure of the nuclear envelope.................................... 32

1.10.2 Resident proteins of the INM and ONM...........................

1.10.3 Traffic into the nucleus via NPCs................................. 36

1.10.4 Protein trafficking throughout the cell ..............................37

1.11 Hypothesis and objectives of thesis ................................. 40

Chapter 2. Materials and Methods................................................42

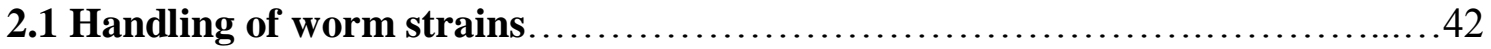

2.2 Genetic crosses..................................................... 42

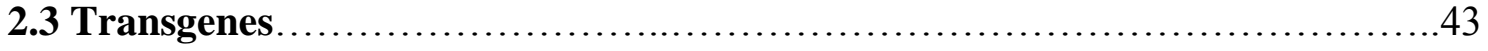

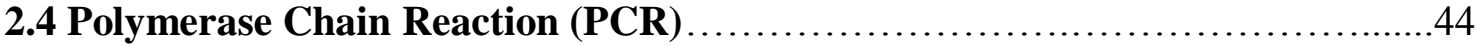

2.5 Scoring of DA and DB motor neuron outgrowth defects..................44 
2.6 Scoring of AVM and PVM mechanosensory neuron guidance defects. .45

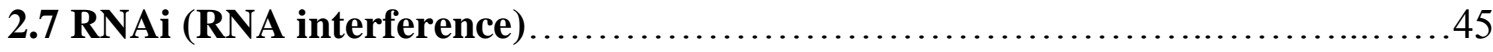

2.8 Seamless cloning for mammalian cell expression ..........................46

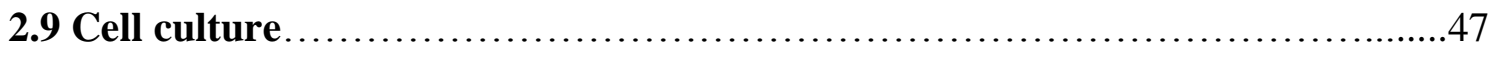

2.10 Transfection and immunofluorescence staining of HeLa cells................47

2.11 Microscopy.......................................................49

2.12 Cell-surface labelling of HeLa cells.......................................49

2.13 Removal of ENU-3's predicted transmembrane domain ...................49

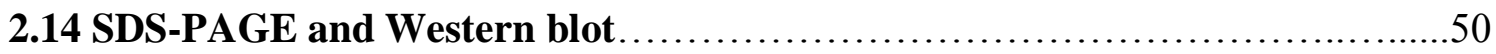

2.15 Construction of reporter constructs and microinjection of $C$. elegans ..........51

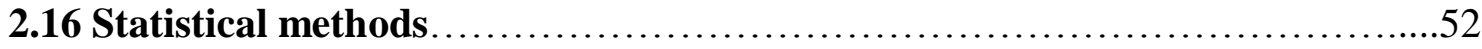

Chapter 3. Results................................................................53

3.1 enu-3 functions in an UNC-6/Netrin dependent pathway parallel to UNC40/DCC/Frazzled to enhance mechanosensory neurons guidance defects........53

3.2 Knockdowns of the paralogs of ENU-3 do not enhance guidance defects of AVM and PVM mechanosensory neurons

3.3 ENU-3 enhances motor neuron outgrowth defects of unc-5 and unc-6

mutants .64

3.4 Motor neuron outgrowth defects are partially dependent on the presence of

UNC-40 .66

3.5 Protein structure of the ENU-3 family members.

3.6 The ENU-3 protein family functions in a parallel pathway to the UNC-6/Netrin pathway to enhance outgrowth defects of motor neurons....

3.7 ENU-3 expresses on the nuclear membrane/ER of HeLa cells .74

3.8 F::ENU-3::GFP and ENU-3::GFP have identical molecular weights...........80

3.9 F::ENU-3 partially co-localizes with ER marker KDEL::GFP...............82

3.10 Removal of the trans-membrane domain of ENU-3 results in secretion of the protein.

3.11 C38D4.1 paralog expresses on the nuclear membrane/ER of HeLa cells......88

3.12 K01G5.3 paralog is a plasma membrane associated protein .................90

Chapter 4. Discussion...............................................................92 
4.1 ENU-3 functions in a pathway dependent on UNC-6/Netrin and parallel to UNC40 to enhance guidance defects of touch receptor neurons.

4.2 ENU-3 and its paralogs function in a parallel pathway to UNC-6/Netrin to enhance motor neuron outgrowth defects .98

4.3 ENU-3 and its paralogs may have roles in cytoskeletal rearrangement. .106

4.4 ENU-3 interacts with ZEN-4 and GEI-4.

4.5 Future experiments.

4.5.1 Localization experiments

4.5.2 ENU-3 family members' expression patterns in C. elegans. 112

4.5.3 Determining the directionality of ENU-3 in dorsal-ventral guidance......113 4.5.4. Determining if ENU-3 and its paralogs function in the Wnt pathway.....114 4.5.5 Testing K01G5.3's ability to modify actin dynamics. 114

4.5.6 Yeast-Two-Hybrid Screen

Chapter 5. Conclusions...........................................................116

Chapter 6. Appendices............................................................118

6.1 Generation of ENU-3, C38D4.1 and K01G5.3 epitope tagged constructs. 118

6.2 Generation of $\Delta T M F:: E N U-3:: G F P$ 123

6.3 Generation of the mec7::ENU-3::GFP construct for microinjection

Chapter 7. References. 


\section{$\underline{\text { List of Tables }}$}

Table 2.1: Integrated transgenes and constructed extrachromosomal arrays. .43

Table 3.1: Mechanosensory neurons migration defects are enhanced by enu-3 57

Table 3.2: RNAi knockdown of the ENU-3 paralogs did not enhance guidance defects of mechanosensory neurons.

Table 3.3: UNC-40 and enu-3 enhance motor neuron outgrowth defects...............65

Table 6.1: List of primers used to generate epitope tagged versions of ENU-3, C38D4.1, K01G5.3, and to delete the trans-membrane (TM) domain of ENU-3.

Table 6.2: Components of PCR mixture to amplify pcDNA3 and the three paralogs.

Table 6.3: PCR settings used to amplify pcDNA3 and the three paralogs.

Table 6.4: List of primers used to generate the mec7::enu-3::gfp construct.

Table 6.5: PCR components to amplify GFP, the promoter and gene of enu-3, and the mec7 promoter

Table 6.6: PCR settings to amplify GFP, the promoter and gene of enu-3, and the mec7 promoter. 125

Table 6.7: PCR components for the fusion mixture of enu-3::gfp and mec7::епи-3::gfp. 125

Table 6.8: PCR settings used to generate enu-3::gfp and the mec7::enu-3::gfp fusion constructs 


\section{List of Figures}

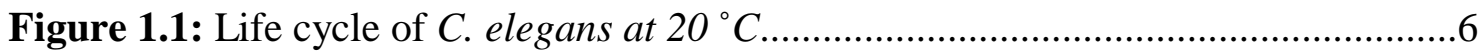

Figure 1.2: Structure of neuronal cells and transmission of signal..................

Figure 1.3: C. elegans nervous system.................................... 13

Figure 1.4: Sensory neurons schematic in C. elegans............................. 14

Figure 1.5: DA and DB motor neurons schematic in C. elegans.....................14

Figure 1.6: Developing neuron growth cone................................. 18

Figure 1.7: UNC-6 signalling in C. elegans.................................21

Figure 1.8: Sources of guidance cues in C. elegans.............................25

Figure 1.9: enu-3 enhances outgrowth defects in $u n c-5$ mutants...................28

Figure 1.10: The expression pattern of ENU-3::GFP in C. elegans................28

Figure 1.11: Predicted structure of ENU-3 using SMART database. ..................29

Figure 1.12: Alignment of the amino acid sequence of the ENU-3 protein family ......31

Figure 1.13: Nuclear envelope structure.................................... 34

Figure 1.14: Traffic pathways throughout the cell............................... 39

Figure 3.1: The mechanosensory neurons are affected by mutations in ENU-3

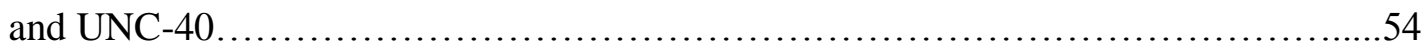

Figure 3.2: RNA interference knockdown of ENU-3 and UNC-40 levels enhance AVM

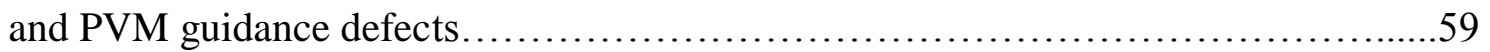

Figure 3.3: Predicted structure of ENU-3 family members using SMART database...68

Figure 3.4: RNAi knockdown of the ENU-3 protein family enhances motor neuron outgrowth defects in $u n c-5(e 53)$ mutants........................................ 
Figure 3.5 RNAi knockdown of the ENU-3 protein family enhances motor neuron outgrowth defects of enu-3(tm4519);unc-6(ev400) mutants........................72

Figure 3.6: RNAi knockdown of the ENU-3 protein family enhances motor neuron outgrowth defects in $u n c-40(e 1430) ; u n c-5(e 53)$ mutants...........................73

Figure 3.7: FLAG::ENU-3 is expressed in the ER and nuclear membrane in HeLa

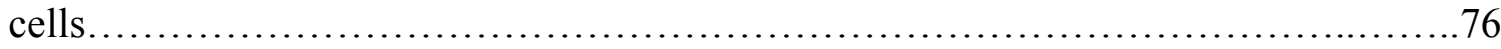

Figure 3.8: FLAG::ENU-3::GFP is expressed in the ER and nuclear membrane in HeLa

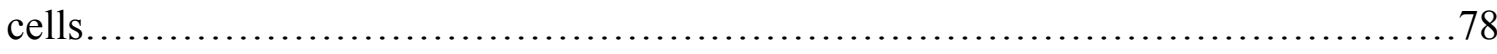

Figure 3.9: ENU-3::GFP is expressed in the ER and nuclear membrane in HeLa cells..79

Figure 3.10: Western Blot anti-GFP confirms the molecular weight of ENU-3........81

Figure 3.11: F::ENU-3 co-localizes with KDEL::GFP on the ER of HeLa cells........84

Figure 3.12: Brefeldin A inhibits secretion of $\triangle \mathrm{TM} F:: E N U-3:: G F P$ from the ER $\ldots \ldots . .87$

Figure 3.13: F::C38D4.1 is expressed in the ER and nuclear membrane in HeLa cells..89

Figure 3.14: Expression pattern of F::K01G5.3 in HeLa cells.......................91

Figure 4.1: Ventral guidance signalling model in $C$. elegans.......................95 


\section{$\underline{\text { List of Appendices }}$}

6.1 Generation of ENU-3, C38D4.1 and K01G5.3 epitope tagged constructs............118

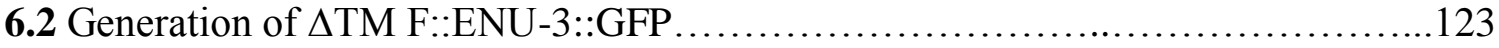

6.3 Generation of the mec $7::$ ENU-3::GFP construct for microinjection................123 


\section{Chapter 1. Introduction}

\subsection{General introduction and outline of thesis}

During the development of the nervous system, individual neuronal cell bodies project their axons towards their final targets, mediated by an interaction between instructive guidance cues and their respective receptors. Growth cones at the tips of the axons of developing neurons express several receptors which can be either chemoattracted or chemo-repulsed by gradients of guidance cues in the extracellular environment. The highly stereotypical and intricate patterns involving growth, migration and development are followed by approximately one billion human neuronal cells and allow for connections even with distantly located cells. The factors that contribute to the directionality of developing neurons are still poorly understood. For instance, the identity of all the molecular cues involved in the instructive guidance of axons remains incomplete. Additionally, the interface between these cues and their receptors as well as how these interactions allow cells to make decisions regarding their correct migration pathways during development, are challenging aspects of the nervous system that are still under investigation. It is important to note that vertebrates and invertebrates share highly similar axon guidance signalling pathways, although the details may vary. Thus $C$. elegans, in which individual neurons can be observed in vivo in a simple nervous system and with a well understood genome, is the ideal model organism for nervous system analysis.

In C. elegans, the Netrin/UNC-6 signalling pathway is responsible for the guidance of axons that migrate in the dorso-ventral axis of the animal (Hedgecock et al., 1990, Ishii et al., 1992). Two receptors expressed on the growth cones of these axons, UNC-40 in 
C. elegans and DCC in humans, and UNC-5/UNC-5 (naming of this gene is the same in C. elegans and humans), which sample the extracellular environment and detect the gradient distribution of UNC-6 in C. elegans and Netrin in humans, along the ventral nerve cord (Chan et al., 1996, Leung-Hagesteijn et al., 1992). UNC-40 is chemo-attracted to sources of UNC-6, while UNC-5 is chemo-repulsed by sources of UNC-6 (Hedgecock et al., 1990). Since null mutants of each of these proteins do not exhibit axon guidance defects in all neurons, this suggests that there may be other signalling pathways involved in dorso-ventral guidance of axons which function redundantly with the Netrin/UNC-6 pathway.

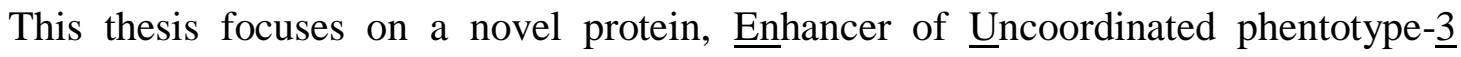
(ENU-3) that was recently found through a genetic enhancer screen for enhanced motor neuron axon outgrowth defects conducted in an unc-5 null background, which resulted in more severely uncoordinated animals (Yee et al., 2011). Although, enu-3 mutant animals do not exhibit any outgrowth defects, animals with mutations in both enu-3 and unc-5 appear to have an enhanced number of axons which do not leave the cell body compared to the unc-5 mutant animals (Yee et al., 2011). ENU-3 is expressed pan-neuronally throughout the nervous system of $C$. elegans, as well as in the cell bodies of motor neurons along the ventral nerve cord (Yee, 2011).

While ENU-3 has been identified as having an important role in axon outgrowth and guidance, the genetic pathways in which it functions, as well as the molecular and cellular mechanisms it utilizes are largely unknown. Hence, the work presented in this thesis seeks to combine genetic, cellular and molecular approaches in several model systems to obtain unprecedented understanding of the function of ENU-3 in motor neuron 
outgrowth. Additionally, its role in controlling axon guidance in C. elegans was an additional aspect of my work described in this thesis. ENU-3 is the founding member of the ENU-3 protein family, which consists of five additional paralogs in the C. elegans genome. They are all of unknown function, with $90 \%$ amino acid sequence identity to ENU-3 (Yee et al., 2011). Therefore, their characterization was also of interest in this thesis. ENU-3 and four of its paralogs are predicted to contain a signal peptide, a transmembrane domain and a nuclear localization sequence (Yee et al., 2014), which led to the investigation of their functionality through expression analyses in HeLa cells. Expressing ENU-3 and its paralogs in mammalian HeLa cells contributed to a better understanding of their roles, as individual subcellular compartment localization was easily identified in these larger cells than the cell bodies of the motor neurons in $C$. elegans.

Chapter 1 provides an introduction to the C. elegans nervous system, and the signalling pathways, conserved among vertebrates and invertebrates. These are known to be involved in axon guidance and axon outgrowth of two classes of neurons, the motor neurons and mechanosensory neurons. Additionally, it provides a brief introduction to the mammalian nuclear envelope structure, nuclear membrane resident proteins and traffic pathways within the cell. Chapter 2 provides all materials and methods used in this study. Chapter 3 reveals all findings of this work. Finally, chapter 4 analyzes and interprets the results, and future experiments are suggested. 


\subsection{Caenorhabditis elegans as a model organism}

Caenorhabditis elegans (C. elegans) is a well characterized free living soil nematode that has been used as a model organism to extensively study molecular mechanisms and protein function of higher eukaryotes. The $C$. elegans genome has been sequenced and approximately 19,000 genes were identified, of which over $40 \%$ were highly homologous to proteins in the Nematoda phylum, fungi, bacteria, and Homo sapiens ( $C$. elegans sequencing consortium, 1998). Most animals are self-fertilizing hermaphrodites but there are males that allow for broad genetic studies that follow Mendelian principles. A typical adult hermaphrodite is $1 \mathrm{~mm}$ in length and its generation time is usually 3 days at $20^{\circ} \mathrm{C}$ resulting in an average of 300 progeny (Brenner, 1974). Laboratory growth and handling of C. elegans is facile as the nematodes are grown on solid liquid broth (LB) medium in standard NGM plates at $20^{\circ} \mathrm{C}$ seeded with the OP50 strain of $E$. coli for short term storage or $16^{\circ} \mathrm{C}$ for medium term storage. The nematodes are inexpensive to maintain as they only feed on E.coli, cholesterol and trace amounts of metals. The handling techniques can be altered based on the application required (Brenner, 1974).

C. elegans mature through four larval stages (L1-L4) after hatching as an egg (Figure 1.1). Once the adult stage has been reached, wild-type hermaphrodites can live for approximately three weeks (Brenner, 1974). Typically, they follow their normal life cycle when favourable conditions are provided, such as ambient temperatures close to room temperature and plentiful amounts of food. In times when favourable living conditions are not met, the nematodes can switch to an alternative life cycle phase after L2, termed the dauer phase, in which they can survive for up to four months. During this time, only essential metabolic processes are functioning in the starved animals. The transition from 
this phase to their typical life cycle phase will occur when the nematodes are exposed to suitable living conditions once again (Brenner, 1974).

Sydney Brenner was a pioneer in the introduction of C. elegans as model organisms for genetic studies given their rapid generation time and large offspring numbers (Brenner, 1974). Since then, the complete cell lineage and neuronal interactions in $C$. elegans have been mapped (White et al., 1986). C. elegans are diploid nematodes and the genome was sequenced entirely in 2002 consisting of five autosomal chromosomes and one sex chromosome (X) (Brenner, 1974) which encode 19,735 protein-coding genes (Hillier et al., 2005). Online tools such as WormBase (www.wormbase.org) provide updated $C$. elegans genetic maps. The Worm Knockout Consortium was able to identify the roles of many worm proteins, through the generation of deletion mutants corresponding to every gene, by utilizing a reverse genetics approach (www.wormbase.org). Since then, various techniques have been used in order to generate mutations and create mutants for specific novel genes. Examples include UV, EMS (ethyl methyl sulfonate), TMP (trimethyl phosphate) and transposon induced mutagenesis. These techniques facilitate genetic analysis through mutation-based screens. 


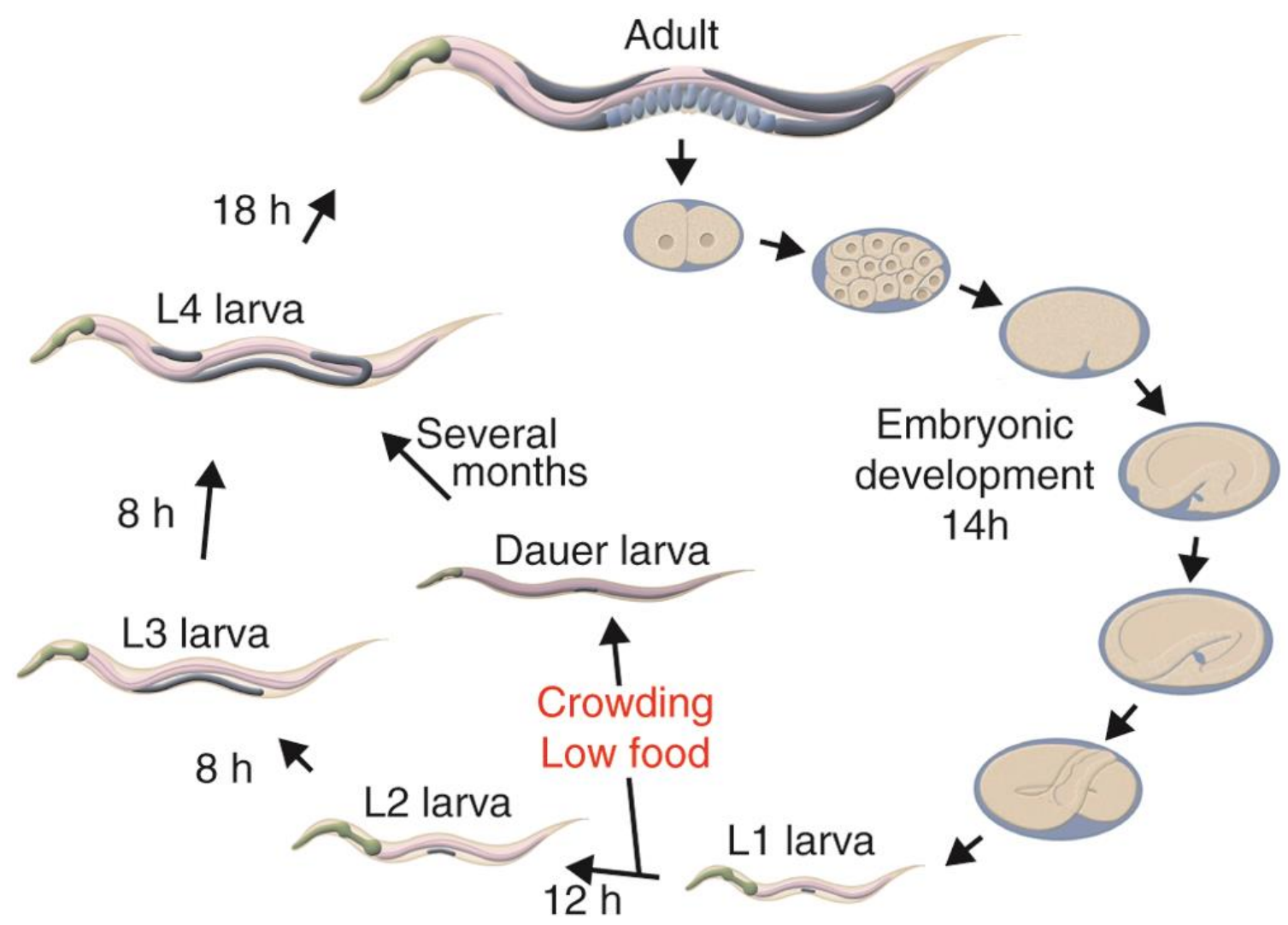

Figure 1.1: Life cycle of C. elegans at $20^{\circ} \mathrm{C}$.

After the egg is released from the vulva, it undergoes a 14-hour embryogenesis period before the egg hatches and the nematode becomes an L1. If favourable conditions are met, L1 matures into L2, followed by L3 and L4 before developing into an adult. An alternate phase can be entered before L2, the Dauer phase, if suitable living conditions are not satisfied, including crowding and low supplies of food. The Dauer larva, which can survive for several months, can re-enter the life cycle as an L4 once living conditions are improved (Reproduced from Murgatroyd \& Spengler, 2010; Hipolito, 2014). 


\subsection{Neuron structure and function}

The highly intricate nervous system of humans contains over one thousand neuronal synaptic connections shared between over three trillion individual neuronal cells (Itzkovitz, Baruch, Shapiro \& Segal, 1992). A neuronal cell contains dendrites, a cell body, an axon and synaptic terminals as shown in Figure 1.2. Each cell is able to receive a signal and transmit it to the next cell through the release of neuro-transmitters at the synaptic cleft. Initially, the neuron is able to receive input through its highly branched dendrites. Consequently, an ionic differential potential is generated which in turn creates an action potential that begins at the axon hillock and is sent throughout the elongated axon. An action potential is known as a self-propagating, unidirectional electrical potential that is triggered only when the difference in membrane potential of the axon surpasses a certain threshold (Palani et al., 2012). Once the signal reaches the synaptic terminals where the cell innervates the target cell to which the signal is passed on, neurotransmitters are released via exocytosis of pre-formed vesicles out of the cell and into the synaptic cleft (Palani et al., 2012).

Upon stimulation of the pre-synaptic cell, this junction between the transmitting neurons and the receiving, target cell becomes locally enriched in specific neurotransmitters that in turn elicit regulated responses in the target, post-synaptic cell (Kennedy, 1998). Distances between the neuron cell body (or soma which contains the nucleus and specialized organelles including the mitochondria, Golgi apparatus, endoplasmic reticulum, secretory granules, and ribosomes) and their target cells can be more than 1 metre long in humans which is spanned by a cell's axon (Murgatroyd \& Spengler, 2010). In order to establish the position of the axonal projection onto the 
correct target cell(s), molecular guidance cues play a crucial role in determining the accurate trajectories of axons and thus mediate the development of neuronal connectivity. 


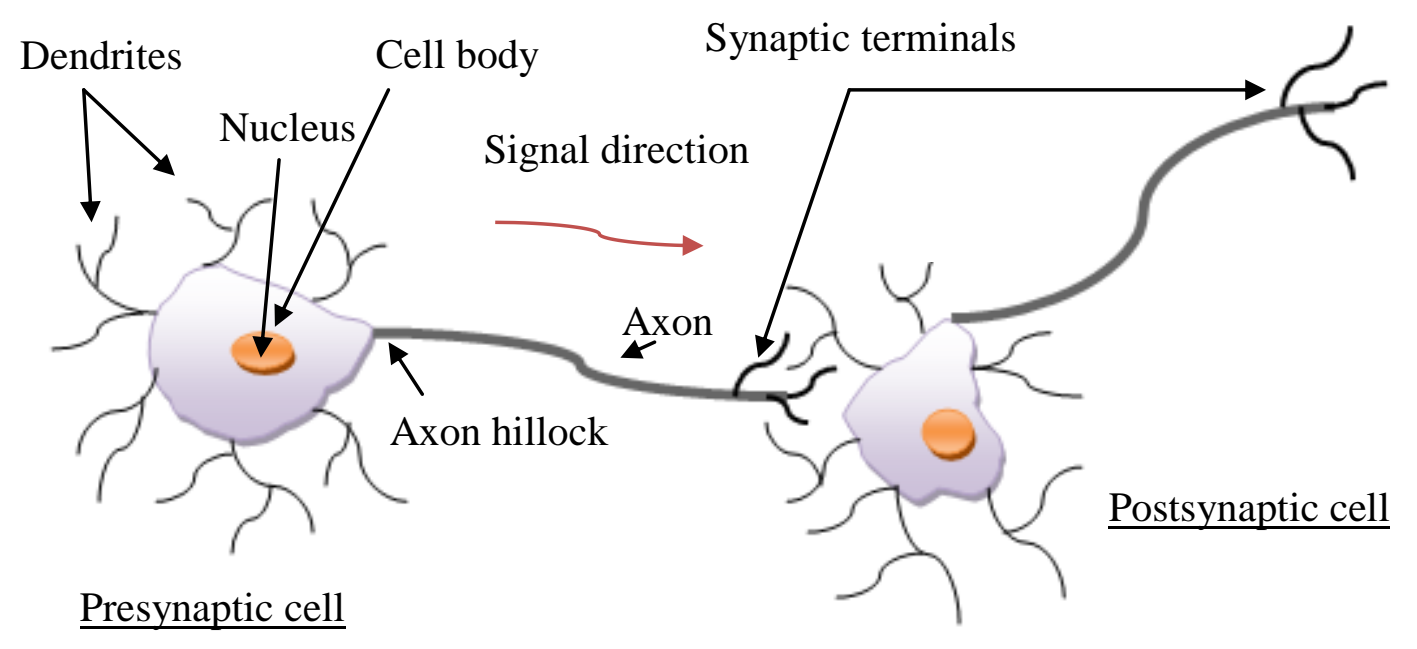

Figure 1.2: Structure of neuronal cells and transmission of signal

A typical neuron consists of dendrites, cell body, axon and synaptic terminals. The presynaptic cell receives signal input via its dendrites and an action potential is initiated at the axon hillock. After it migrates through the entire axon, neuro-transmitters are released at the synapse with the target, post-synaptic cell. The binding of neurotransmitters to their respective receptors on the post-synaptic cell results in further signal conduction, ultimately leading to regulation of a response in the target cell. 


\subsection{C. elegans nervous system}

Since all individual neurons and their synaptic connections have been identified in C. elegans (White et al., 1986), these nematodes are considered ideal model organisms for visualizing single neurons and understanding the molecular mechanisms underlying axon guidance and growth. There are 302 neurons in an adult hermaphrodite and 381 neurons in an adult male, with most of the additional neurons contributing to malespecific mating behaviour. This behaviour is responsible for sensing hermaphrodites and attaching its tail to them while searching for the vulva where the male can insert its spicules and release its sperm into the hermaphrodite (Sulston, Albertson, \& Thomson, 1980). As shown in Figure 1.3, there are two types of neurons. Commissural neurons send their axons circumferentially along the body of the animals, while longitudinal axons send their projections along the anterior-posterior axis of the animals. Of these, three major classes of neurons are identified: sensory neurons, motor neurons and interneurons (White et al., 1986).

The majority of neurons are in close proximity primarily in the nerve ring, a structure that is found around the pharynx, and is usually referred to as the brain of the animal, where many neuronal connections occur. Neurons are also found along the two major longitudinal nerve cords, the dorsal nerve cord (DNC) and ventral nerve cord (VNC). Along the VNC, axons of several sensory neurons, 57 motor neurons and various interneurons are found (White et al., 1986).

Mechanosensory touch receptor neurons are the type of neurons that have the ability to sense the extracellular environment, including chemical gradients and temperature, through distinct sensory dendrites. Furthermore they are able to regulate responses to 
these cues such as the formation of correct trajectories of developing neurons (Hall, Lints, \& Altun, 2005). The six mechanosensory neurons in C. elegans are the anterior ventral microtubule (AVM), the posterior ventral microtubule (PVM), two anterior lateral microtubules (ALM) and two posterior lateral microtubules (PLM) on the left and right side of the animals. The cell bodies of AVM and PVM are located along the midline and the axons are sent ventrally to the $\mathrm{VNC}$, followed by a turn towards the head of the animal, while the ALMs and PLMs have processes that run directly towards the head along the mid-body (Hall, Lints, \& Altun, 2005), (Figure 1.4).

Motor neurons are divided in eight subclasses: DA, DB, DD, AS, VD, VA, VB, VC, and are responsible for forward and backward movement by innervating body wall muscles through neuromuscular junctions (Chalfie et al., 1985). Figure 1.5 shows that the cell bodies of DAs and DBs are located along the ventral nerve cord, and their axons are sent circumferentially towards the dorsal nerve cord, where they turn their processes and migrate either towards the anterior side or the posterior side along the DNC innervating dorsal body wall muscles (White et al., 1986). Additionally, DD, DA and DB subclasses of motor neurons are established during the embryogenesis phase of the life-cycle, while the others are formed during L1 (White et al., 1986). The VA, BV, VC and VD subclasses of motor neurons send their processes ventrally and innervate ventral body wall muscles (White et al., 1986). It was identified that DA and VA excitatory motor neurons are responsible for backward movement, while DB and VB excitatory motor neurons result in forward movement, through the use of laser ablation experiments (Chalfie et al., 1985). Furthermore, the DD and VD inhibitory motor neurons are responsible for muscle relaxation. The cholinergic motor neurons are represented by the 
A- and B-class of motor neurons, while the D-type motor neurons are GABAnergic (Hall \& Altun, 2008). 


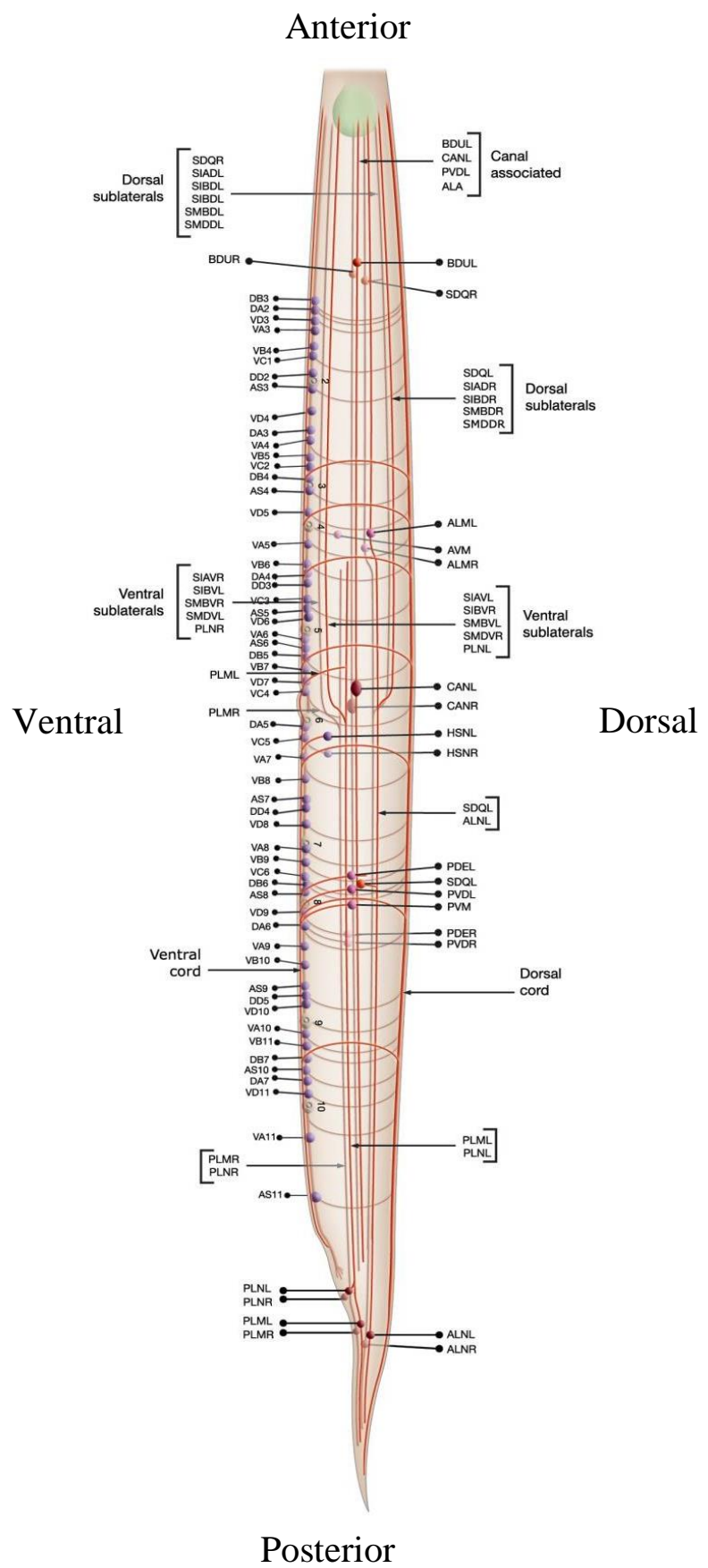

Figure 1.3: C.elegans nervous system

A subset of neurons in the nervous system of $C$. elegans is shown. Commissural neurons have their cell bodies located along the $\mathrm{VNC}$ and send their axons from the ventral nerve cord to the dorsal cord. Longitudinal nerve tracts extend along the body of the nematode from the anterior side to the posterior side of the animal (White et al., 1986). 


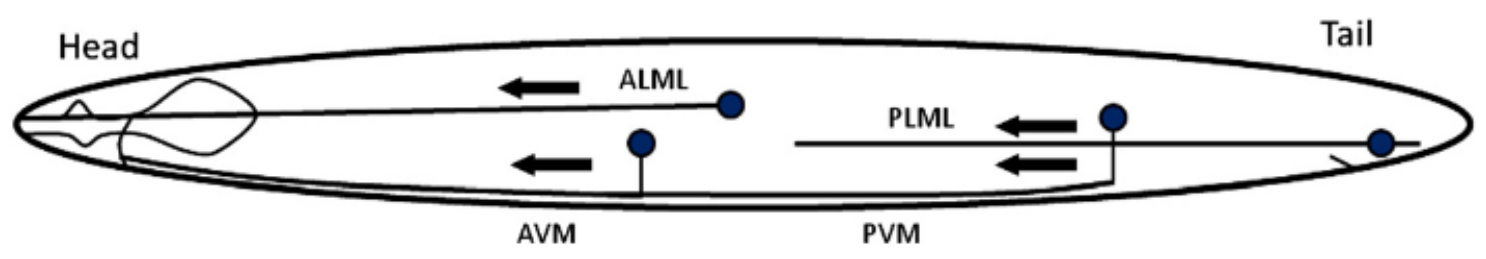

Figure 1.4: Sensory neurons schematic in C. elegans

The AVM on the right side of the body of the animal and the PVM on the left side of the body of the animals send their processes to the $\mathrm{VNC}$ where they turn towards the head of the animal, migrating along the VNC. The ALM and PLM on the left side (ALM and PLM on the right not shown) of the body of the animal send their axons longitudinally along the mid-line of the animal towards the head (Retrieved from Killeen \& Sybingco, 2008).

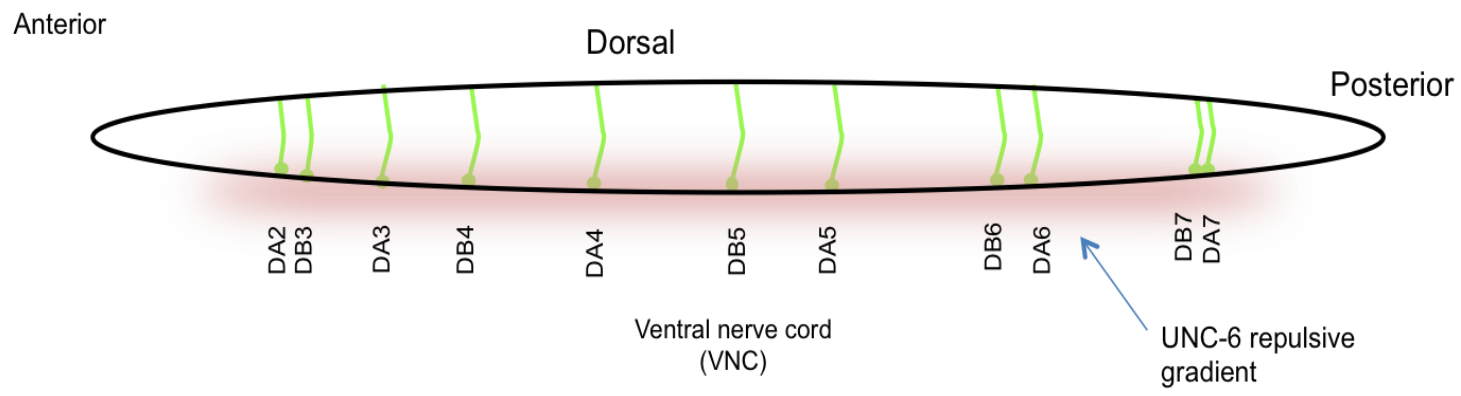

Figure 1.5: DA and DB motor neurons schematic in C. elegans

The DA and DB motor neurons cell bodies are aligned along the VNC during embryogenesis and send their processes circumferentially to the dorsal cord where they innervate the dorsal body wall muscles. DAs regulate backward motion while DBs regulate forward motion in C. elegans (Image retrieved from Hipolito, 2014). 


\subsection{Growth cones, axon outgrowth and axon guidance}

The correct wiring of the complex nervous system is essential for proper brain function. Growth cones are dynamic, finger-like structures found at the leading edge of the migrating axon that can sense molecular cues in the extracellular environment which allow it to grow and migrate in the correct trajectory during its development (Maskery \& Shinbrot, 2005). Once the axon reaches its target, these structures disappear and are replaced by synapses. The axonal cytoskeleton consists of microtubules identified as the central domain and of actin filaments abundantly present in the peripheral domain (Goldberg, 2003). Growth of the leading edge of the axon is mediated by actin polymerization, in which actin monomers accumulate through constitutive plus enddirected filament assembly (Goldberg, 2003). Consequently, the filaments in the tips of filopodia and lamellipodia reach outward thus pulling the central domain of the growth cone forward resulting in axon growth. Concurrently, myosin molecular motors induce actin filament depolymerisation by pulling the actin filament back towards the central domain (Goldberg, 2003). The movements caused by myosin machinery function to hinder microtubules from growing into the peripheral domain which in turn prevents the forward movement of the central domain (Goldberg, 2003), and the axon cannot grow. Thus, axon outgrowth is determined by a balance between plus end-directed actin polymerization and myosin-induced retraction. Increased actin polymerization at the leading growth cone edge, and supressed myosin activity, permitting microtubule polymerization, allow forward growth and movement of actin filaments, thereby resulting in axon elongation (Goldberg, 2003). 
Regulation of the mechanism of axon growth is established by proteins involved in enhancing plus-end actin polymerization or inhibiting myosin-induced retrograde movement of axons. Profilin is a protein known to bind to monomers of actin and is involved in enhancing actin polymerization (Goldberg, 2003). The Enabled family of proteins recruit Profilin to the growth cone where it promotes higher levels of plus enddirected polymerization leading to axon outgrowth (Goldberg, 2003). Numerous other extracellular signals have also been involved in promoting axon growth, most notably peptide trophic factors such as neurotrophins (Bibel \& Barde, 2000). In mammals, nerve growth factor (NGF) and brain-derived neurotrophic factor (BDNF) have been proven to promote axon outgrowth and thus play a role in the development of the nervous system (Bibel \& Barde, 2000). In addition, it has been identified that the combination of multiple trophic factors, namely NGF and BDNF, cause greater axon outgrowth than any of them alone in dorsal root ganglion neurons, however different neurons respond to different trophic factors (Lindsay, Thoenen \& Barde, 1985). Moreover, filamentous structures such as filopodia and lamellipodia (Figure 1.6), which along with the expression of certain receptors guide the migration of axons through gradients of molecular cues in the direction in which they must develop (reviewed in Vitriol and Zheng, 2012).

Previous research has established several pathways involved in axon guidance and it appears as if most of the machinery necessary for navigation of axons during development of neural circuitry is present in the growth cones (Shaw \& Bray, 1977, reviewed in Vitriol and Zheng, 2012). The major classes of axon guidance cues that have been studied are the Netrins, Slits, Ephrins and Semaphorins along with their receptors (reviewed in Killeen \& Sybingco, 2008, Kolodkin \& Tessier-Lavigne, 2011). In addition, 
some morphogenic factors such as Shh, Wnts VEGF and BMPs (TGF $\beta$ ) have axon guidance activity (Yam \& Charron, 2013). Alterations of calcium levels are important in growth cone guidance (Tojima et al., 2012). Many axons are simultaneously subjected to more than one cue. The mechanisms in which various signals are integrated are the subject of intense recent research (reviewed in Dudanova \& Klein, 2013).

One major class of secreted guidance cue, UNC-6/Netrin, expressed as a gradient along the VNC, can control short-range and long-range migrations of axons that can be either chemo-attracted or chemo-repulsed by this source (Hedgecock et al., 1990, Ishii et al., 1992, reviewed in Killeen \& Sybingco, 2008 and in Lai et al., 2011). Two other classes of molecular cues involved in guidance of axons are the Semaphorins and Ephrins which regulate repulsion of short-range migration and thus deter axons migration from specific areas (Wang et al., 1999; Wong et al., 1997). In C. elegans, three important proteins, UNC-6, UNC-40 and UNC-5, have been identified to contribute to axon migration in the dorso-ventral axis, which constitute the Netrin/UNC-6 signalling pathway (Hedgecock et al., 1990, Ishii et al., 1992, Chan et al., 1996, Leung-Hagesteijn et al. 1992). 


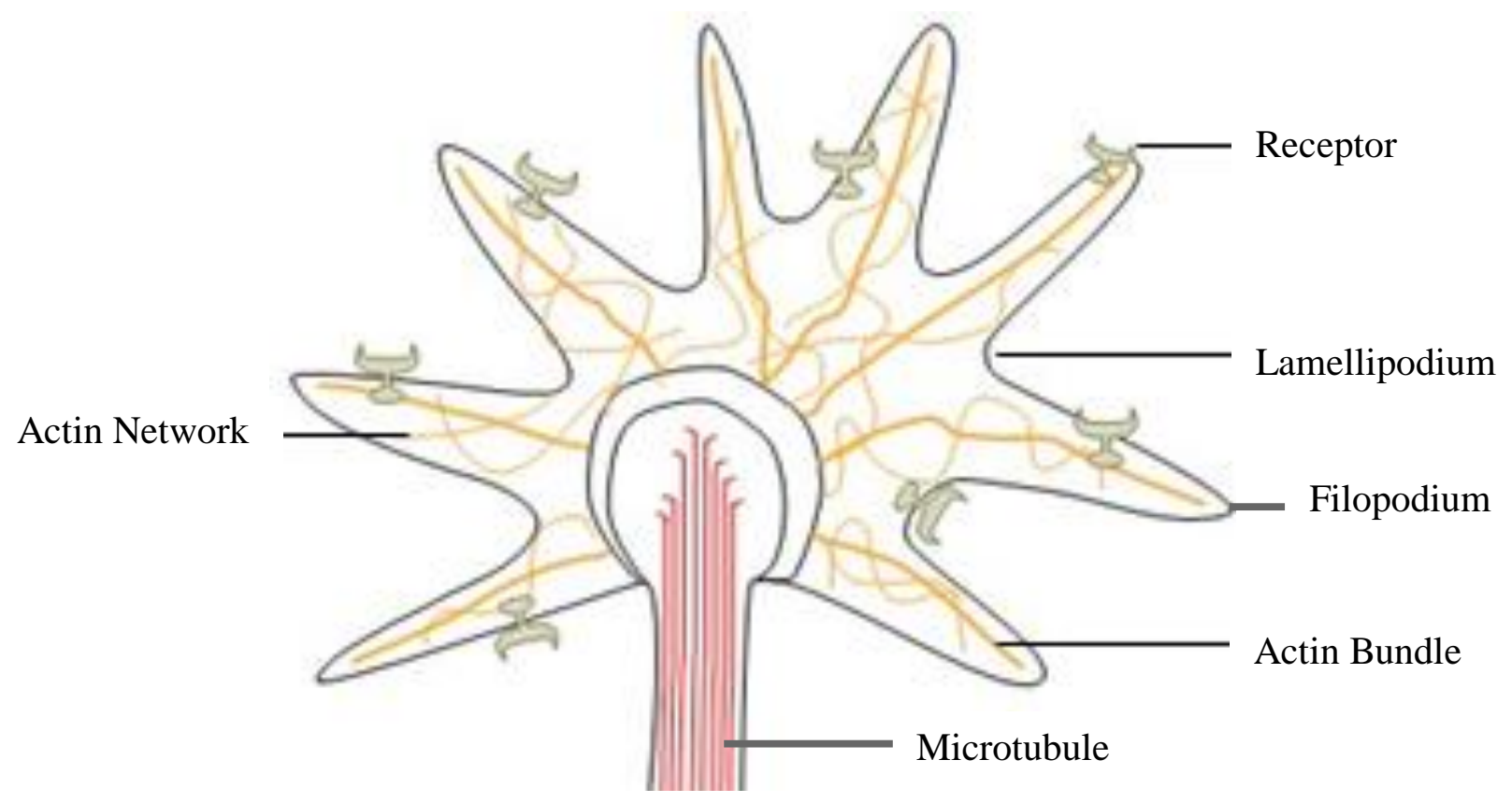

Figure 1.6: Developing neuron growth cone

The tip of the developing neuron, the growth cone, extends filamentous projections to sample the environment through interactions between receptors expressed on the growth cone and molecular cues in the extra-cellular environment. The instructive roles of these cues physically guide the axons and promote proper trajectories of neurons (Adapted from Hipolito, 2014). 


\subsection{The UNC-6/Netrin signalling pathway}

The conserved signalling pathway between vertebrates and invertebrates which regulates outgrowth and migration of cells and developing neurons is the UNC-6/Netrin pathway (Ishii et al., 1992). UNC-6 and Netrin, its mammalian homologue, are lamininlike molecular cues which are secreted by ventrally aligned guide-post cells and are localized in a gradient fashion along the VNC, decreasing dorsally in $C$. elegans (Ishii et al., 1992). First identified in C. elegans, UNC-6 was found to instructively direct axon migration along the dorso-ventral axis (Hedgecock et al., 1990). Additionally, identical instructive roles for axonal growth were established with UNC-6 homologues in Drosophila (Harris et al., 1996, Mitchell et al., 1996) and the Netrins in vertebrates (Serafini et al., 1994, Kennedy et al., 1994). In C. elegans, the dorsal or ventral migration of axons depends on the interaction between UNC-6 and two receptors, UNC40/DCC/Frazzled and UNC-5 (Chan et al., 1996, Leung-Hagesteijn et al., 1992). In other organisms the UNC-40 receptor is known as DCC (deleted in colorectal cancer) in mammals and Frazzled in Drosophila (Keino-Masu et al., 1996, Hiromoto et al., 2000). In higher organisms the UNC-5 receptor is known as Unc5H1-4 (Leonardo et al., 1997, Ackerman \& Knowles, 1998). Growth cones expressing UNC-40 receptors are chemoattracted by sources of UNC-6, thus will project their axons ventrally, while growth cones expressing UNC-5 receptors are chemo-repulsed by sources of UNC-6, and will project their processes dorsally, away from ventrally expressed UNC-6 (Hedgecock et al., 1990). However, growth cones which express heterodimers of UNC-40 and UNC-5 will also project their axons dorsally, away from sources of UNC-6/Netrin, as it has been shown that the chemo-repulsion between UNC-6 and UNC-5 overcomes the chemo- 
attraction between UNC-6/Netrin and UNC-40/DCC (Hong et al., 1999) (Figure 1.7). Thus, it is evident that the effects of UNC-6 on migrating axons depend on the receptors expressed on the growth cones of developing neurons. Interestingly, the migration of axons over long distances at low UNC-6/Netrin concentrations is more efficient when UNC-40:UNC-5 heterodimers are present rather than UNC-5 homodimers (Keleman \& Dickson, 2001, MacNeil et al., 2009).

Mutations in the genes that encode UNC-6, UNC-5 and UNC-40 all cause axon outgrowth and migration defects, translating into uncoordinated (Unc) phenotypes, typically as a consequence of errors in migration of dorsally directed commissures of motor neurons (Hedgecock et al., 1990). An axon outgrowth defect is observed when axons fail to exit the cell body thus inhibiting motor neuro-muscular junction formation. In this work, the type of defect described is usually an axon outgrowth defect. Alternatively, an axon guidance defect is identified by the processes of motor neurons leaving the cell body but not reaching the dorsal cord, instead migrating mostly one third of the way, where the processes then turn towards the anterior side of the animal. These have been shown to occur in the null unc-5, unc- 6 mutants and to lesser extent in unc-40 mutants (Hedgecock et al., 1990). Similarly, ventral guidance is affected by mutations in UNC-6 and UNC-40, excluding UNC-5. In either of the two mutants, the axons of two mechanosensory neurons, AVM and PVM are projected longitudinally along the mid-line of the animal, and thus do not migrate ventrally to reach the $\mathrm{VNC}$, as it would occur in wild-type animals (Desai et al., 1998). 


\section{Dorsal}

UNC-5 or UNC-40/UNC-5

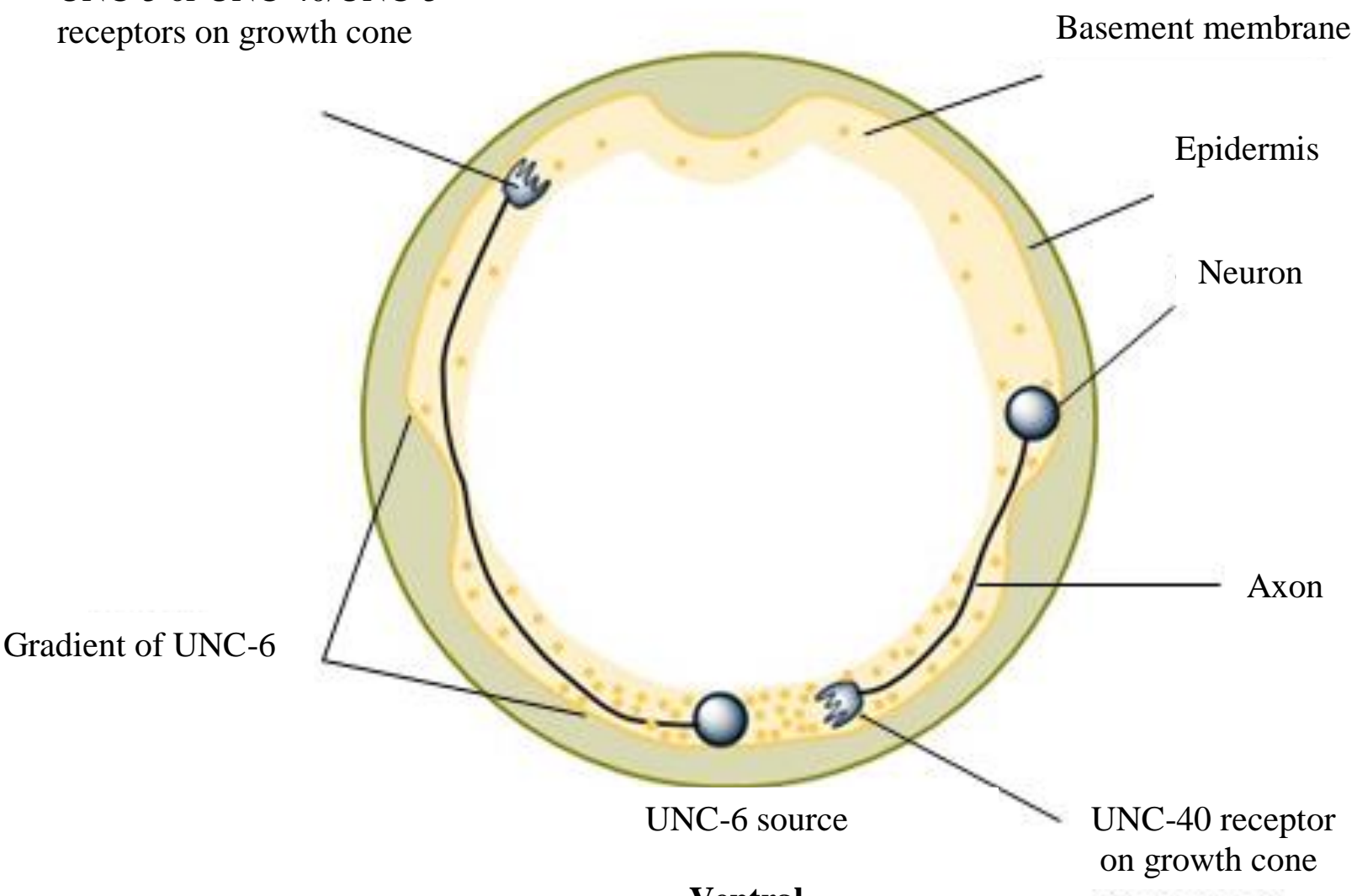

\section{Ventral}

Figure 1.7: UNC-6 signalling in C. elegans

The cross section of the body of C.elegans shows the gradient expression of UNC-6 along the VNC which chemo-attracts axons that express UNC-40 receptors, and chemorepulses axons that express either UNC-5 or UNC-40 and UNC-5 receptors (Modified from Ishii et al., 1992, Wadsworth et al., 2002). 
However, the UNC-6/Netrin signalling pathway is evidently not the sole pathway regulating axon outgrowth and guidance in the dorsal-ventral axis since null mutants lacking UNC-6, UNC-5 or UNC-40 do not exhibit $100 \%$ penetrance of resulting phenotypes, such as uncoordinated animals (Chan et al., 1996). UNC-129/TGF $\beta$ is another gradient cue expressed along the dorsal muscle band which mediates long-range migration of motor neuron axons by chemo-attracting growth cones expressing UNC-5 (Colavita et al., 1998). One role of UNC-129 is to cause dissociation of UNC-5 homodimers in favor of UNC-40:UNC-5 heterodimers (Colavita et al., 1998).

It is important to note that the human homologues of the three proteins involved in the UNC-6/Netrin signalling pathway function in a similar manner. Netrin, expressed along the ventral midline floor plate attracts commissural neurons expressing DCC and repulses trochlear neurons expressing UNC-5H1 (Serafini et al., 1994, Colamarino et al., 1995, reviewed in Killeen \& Sybingco, 2008). This emphasizes the significance of studying the development of the nervous system in C. elegans, with the global intent that new findings will contribute to the current understanding of neurodegenerative diseases in humans (Chen \& Burgoyne, 2012). 


\subsection{Other signalling pathways regulating axon guidance}

In C. elegans, axons generally migrate in the dorsal-ventral axis or the anteriorposterior axis. In addition to the UNC-6/Netrin signalling pathway which mediates axon guidance of mechanosensory neurons, a separate pathway, the SLT-1/Slit pathway also contributes to ventral guidance of the same neurons (Hao et al., 2001). The SLT-1 gradient guidance cue expressed on the dorsal muscle repels axons that express SAX3/Robo (Roundabout) and co-receptor EVA-1 (Zallen et al, 1998, Fujisawa et al., 2007) consequently promoting ventral guidance of touch receptor neurons such as AVM and PVM. Thus, developing AVM and PVM axons migrate ventrally as a result of the balance between the chemo-repulsion of SAX-3 by SLT-1 along the dorsal nerve cord and chemo-attraction between UNC-40 and UNC-6 along the VNC (Hao et al., 2001) (Figure 1.8). In the absence of both SLT-1 and UNC-6, most of the AVMs and PVMs fail to migrate in the ventral direction and instead migrate directly towards the head.

On the other hand, the only known pathway guiding the motor neuron axon migration towards the dorsal cord is the UNC-6/Netrin pathway (Colavita et al., 1998). The repulsion of UNC-5 by UNC-6 along the VNC, and dorsal gradient sources of UNC129/TGF $\beta$ promotion of UNC-40:UNC-5 heterodimers result in long-range migration of motor neurons permitting the axons to reach the dorsal nerve cord (Colavita et al., 1998, MacNeil et al., 2009) (Figure 1.8).

Although not a focus of this thesis, anterior-posterior migration of axons is affected by sources of SLT-1/Slit at the anterior side of the animal and EGL-20/Wnt sources of guidance cues at the posterior side of the animal (Figure 1.8). Neurons that express Frizzled receptors on their growth cones such as ALM and PLM are repelled by sources 
of Wnts, and thus project their processes towards the head of the animal (Reviewed in Killeen and Sybingco, 2008). 


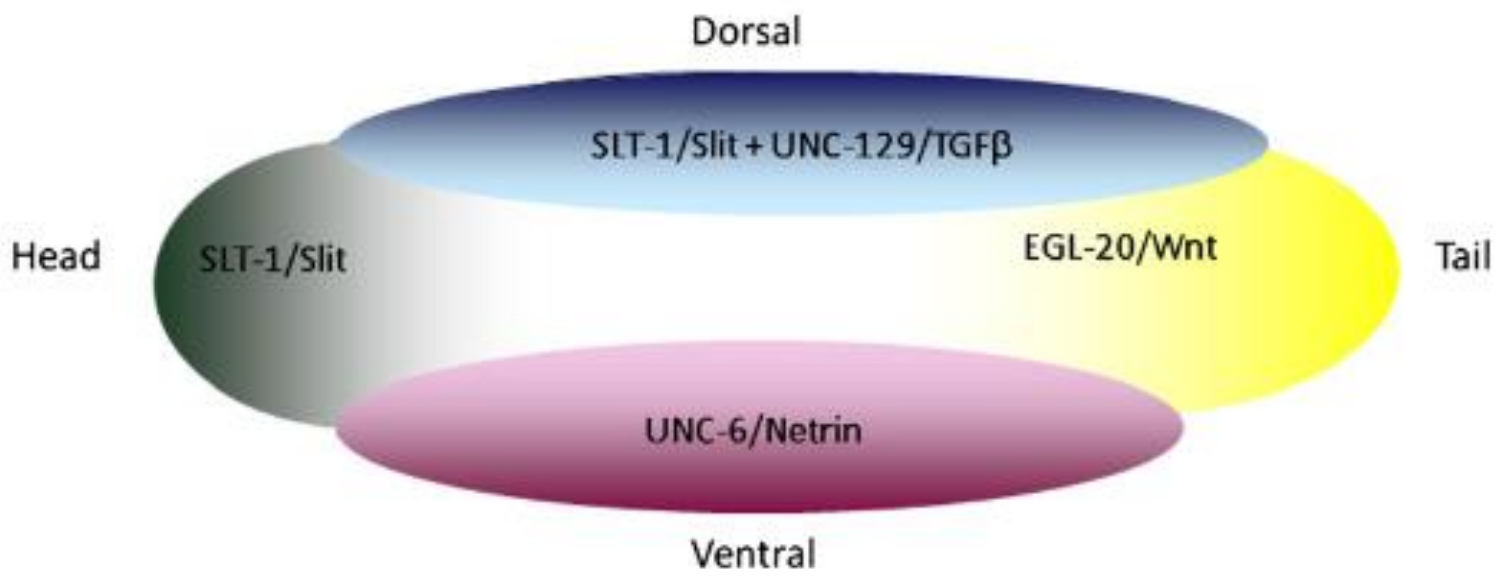

Figure 1.8: Sources of guidance cues in C. elegans

Sources of UNC-6 along the VNC repel axons that express UNC-5 and attract axons that express UNC-40. SLT-1 repels axons that express SAX-3/EVA-1, while UNC-129/TGF $\beta$ promotes formation of UNC-40:UNC-5 heterodimers to promote long-range migration of motor neurons. In the anterior-posterior axis, EGL-20/Wnt sources repel axons that express Frizzled receptors (Killeen and Sybingco, 2008). 


\subsection{Discovery of ENU-3 and its role in the nervous system of C.elegans}

As described previously $C$. elegans mutants lacking either UNC-6 or UNC-5 have motor neuron axon guidance defects such that the motor neurons do not reach the dorsal cord in null mutant animals. These animals are extremely Unc (uncoordinated). However, most axons of the DA and DB classes successfully exit the ventral nerve cord, suggesting the existence of an additional axon outgrowth pathway. In order to define this pathway, a genetic enhancer screen was conducted in an unc-5(e53) putative null background for animals that were more defective in axon outgrowth. This screen resulted in the identification of five new viable mutants (Yee et al., 2011). One mutant identified was

called enu-3 (enhancer of Unc) and one allele enu-3(rql) was characterized. A second allele containing a mutation in the gene affected in rql (H04D03.1) was requested and obtained from the National Bioresource in Japan run by Dr. Mitani called enu-3(tm4519). It was found that enu-3 enhanced the axon outgrowth defects of unc-5 mutants, especially in the DB subclass of motor neurons (Yee et al., 2011) (Figure 1.9).

A translational fusion reporter construct, ENU-3::GFP was micro-injected into $C$. elegans and it was shown that it expressed pan-neuronally with an emphasis in the cell bodies of motor neurons along the VNC (Yee et al., 2011) (Figure 1.10).

H04D03.1/enu-3 is a predicted type I transmembrane protein of 204 amino acids encoded by three exons, weighing $22702 \mathrm{kDa}$ (Yee et al., 2011) (Figure 1.11). SMART database predicted based on ENU-3's amino acid structure that it has a signal peptide domain from amino acid 1 to amino acid 23. Following this site of cleavage, there is a predicted extracellular region from residue 23 to 108 . The putative single pass transmembrane domain spans residue 108 to 128 , followed by a short intracellular region 
up until residue 204. ENU-3 is also predicted to contain several coiled-coil regions. enu-3(tm4519) has a 400 base pair deletion mutation between exon 2 and exon 3 resulting in a shorter version of the protein (Figure 1.11). 

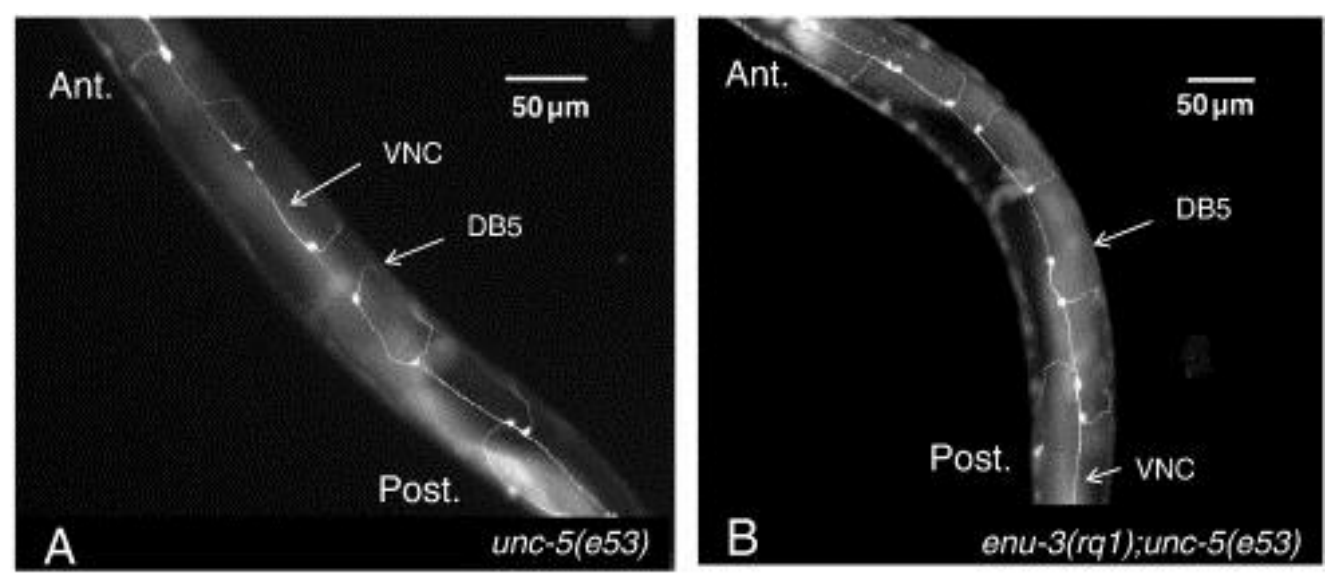

Figure 1.9: enu-3 enhances outgrowth defects in $u n c-5$ mutants

enu-3originally identified as $r q l$ was found to enhance motor neuron outgrowth defects though a genetic enhancer screen in unc-5(e53) mutant background. Compared to the single unc-5 mutant (A), the enu-3(rq1);unc-5(e53) double mutant showed an enhancement of outgrowth defects, especially in DB5 (Yee et al., 2011).

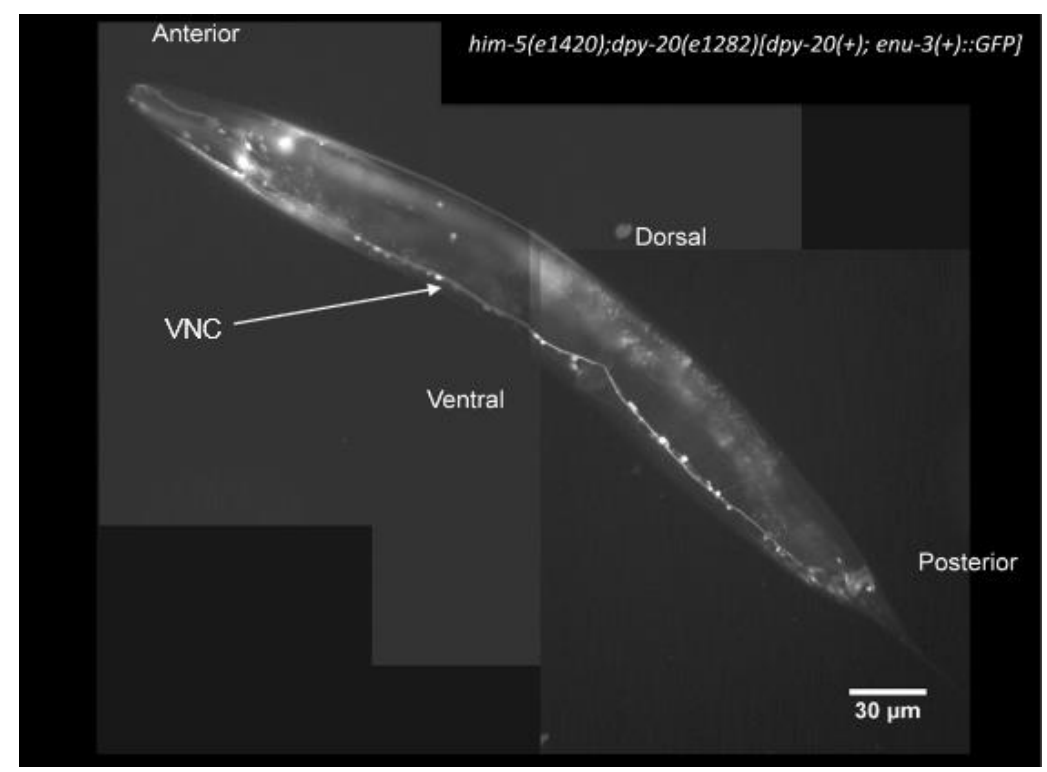

Figure 1.10: The expression pattern of ENU-3::GFP in C. elegans

The translational fusion reporter construct ENU-3::GFP with co-injection marker $d p y-20$ were microinjected into him-5(e1420);dpy-20(e1282) L4 animals, and visualized at x400 total magnification (Yee, 2011). 


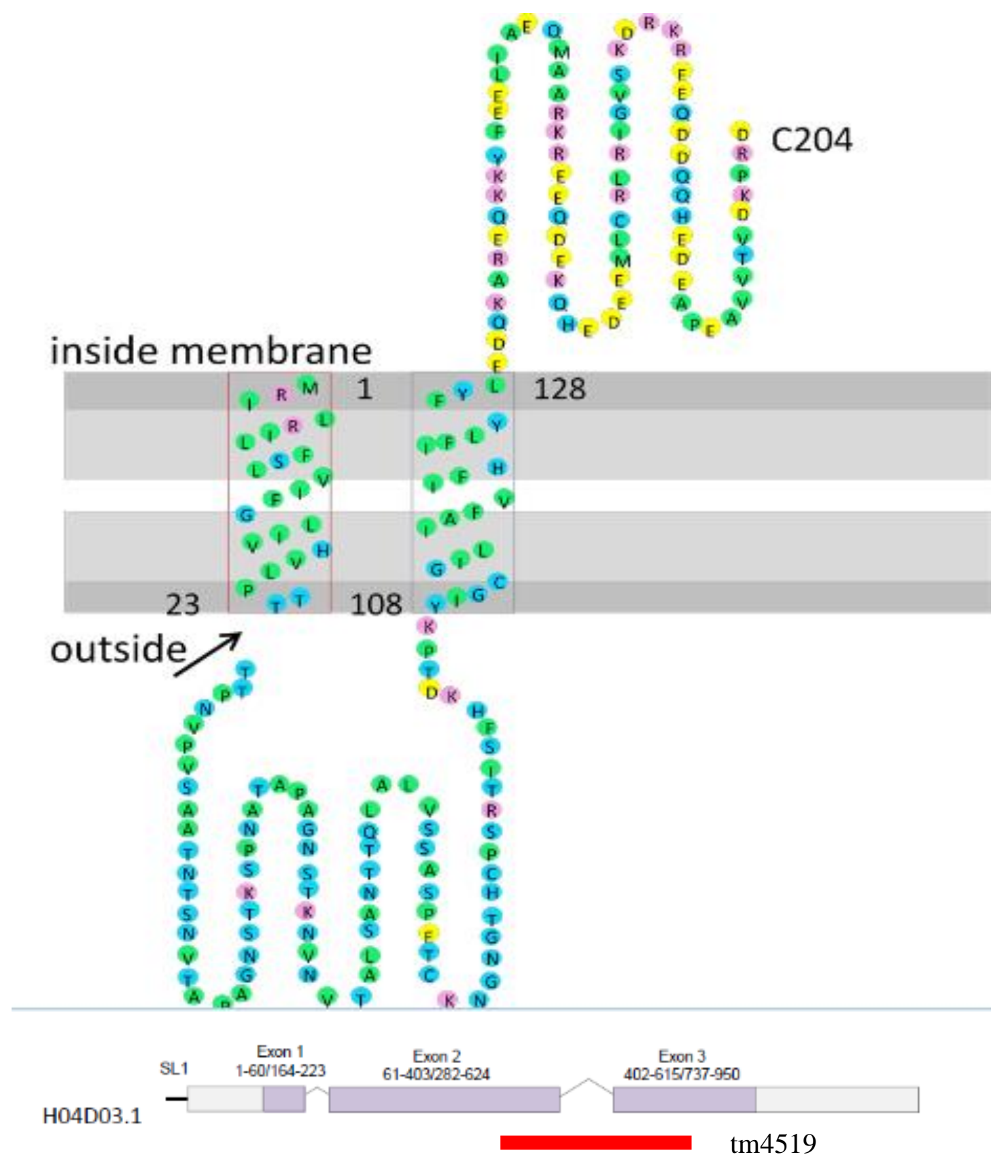

Figure 1.11: Predicted structure of ENU-3 using SMART database.

The key features of ENU-3 predicted by the SMART database are a signal peptide, transmembrane domain and coiled-coil regions (Yee et al., 2011). The red bar represents the deletion mutation of enu-3 (tm4519). 


\subsection{Paralogs of ENU-3}

The C. elegans genome contains genes encoding five additional proteins all of unknown function that are highly homologous to each other and to ENU-3 based on their amino acid sequence alignment. BLAST searches using ENU-3, a 204 amino acid protein, demonstrate that four of the five paralogs are highly similar to ENU-3 but they contain a longer predicted intracellular region and additional exons. C38D4.1, Y37D8A.12, W03G9.3, W05F2.2 contain 462, 458, 462 and 457 amino acids, respectively, and their alignment with ENU-3 (H04D03.1) is shown in Figure 1.12. K01G5.3 with 320 amino acids is highly homologous to the longer intra-cellular region of the other four paralogs, but not to ENU-3 (Figure 1.12). The high degree of similarity and amino acid sequence identity shown in Figure 1.12 indicate possible linked functions of these paralogs and ENU-3, however, nothing is known about the roles of the paralogs in motor neuron axon outgrowth or guidance. 
ENU-3

K01G5.3

ENU-3

K01G5. 3

W05F2. 2

Y37D8A. 12

W03G 9.3

C38D4. 1

ENU-3

K01G5. 3

W0 $5 \mathrm{~F} 2.2$

Y37D8A. 12

W03G9. 3

C38D4. 1

MRILRILFSLVIFGLIVHVLPTTTTPNVPVSAATNTSNVTAPAGNSTKSPNATAPAGNST MRILCILFSLVIFGLIVHVLPTTTTPNVPVSAATNTSNVTAP----TKSPNATAPAGNST MRILRILFSLVIFGLIVHVLPTTTTPNVPVSAATNTSNVTAPAGNSTKSPNATAPAGNST MRILRILFSLVIFGLIVHVLPTTTTPNVPVSAATNTSNVTAPAGNSAKSPNATAPAGNPT

KNVNVTALSANTTQLALVSSASPETCKNGNGTHCPSR--TISFHKDTPKYIGCGILIAFV

----------------------------------------------------------MC KNVNVTAPAANTTQLALVSSASPQTCKNGNGTHCPSCAYTINVHKDTPKYIGYGILIAFV KNVNVTAPAANTTQLALVSSASPQTCKNGNGTHCPSCAYMINVHKDTPKYIGYGILIAFV KNVNVTAPAANTTQLALVSSASPQTCKNGNGTHCPSCAYTINVHKDTPKYIGYGILIAFV KNVNVTAPAANTTQLALVSSASPQTCKNGNGTHCPSCAYTINVHKDTPKYIGYGILIAFV

IFHIFLYFYLEDQKAREQKKYFEELIAEQMAARKREEQDEKQHEDEEMLCRLRIGVSKDR

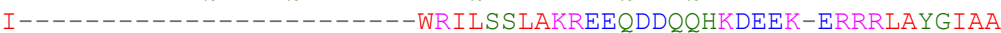
IFHIFLYFYLEDQKAREQKKYFEELIAEQMAARKREEQDDQQHEDEEEDERRG-----TP IFHIFLYFYLEDQKAREQKKYFEELIAEQMAARKREEQDDQQHEDEEKERRERLAYGIAA IFHIFLYFYLEDQKAREQKKYFEELIAEQMAARKREEQDDQQHEDEEKERRERLAYGIAA IFHIFLYFYLEDQKAREQKKYFEELIAEQMAARKREEQDDQQHEDEEKERRERLAYGIAA * . $* * * * * * . * * * * * * * \quad *$

KREEQDDQQHEDE ---------------------------------------------NPYVVEE----IKTPNGNVTKVMEKRRSAVAPPITPALGAGSTNGPYESRTKTAQKEEAM RAYVVAE----SKTSNGNVTKVMEKRRSAVAPPITPALGAGS INGPYESRTKTAQKEEAI NPYVVAE----SKTPNGNVTKVMEKRRSAVAPPITPALGAGSTNGPYESRTKTAQKEEAM NPYVVAE----SKTPNGNVTKVMEKRRSAVAPPITPALGAGSTNGPYESRTKTAQKEEAM NPYVVAE----SKTPNGNVTKVMEKRRSAVAPPITPALGAGSTNGPYESRTKTAQKEEAM $\cdot$

SCHLQVIADNWGRGKELISLINDLDLPAPLVTTEEKARYALELVQHHVEKMTEQKVFLHG SCHLQVIADNWGRGKELISLINDLDLPAPLVTTEEKARYALELVOHHVEKMTEQKVFLHG SCHLQVIADNWGRGKELISLINDLDLPAPLVTTEEKARYALELVQHHVEKMTEQKVFLHG SCHLQVIADNWGRGKELISLINDLDLPAPLVTTEEKARYALELVQHHVEKMTEQKVFLHG SCHLQVIADNWGRGKELISLINDLDLPAPLVTTEEKARYALELVQHHVEKMTEQKVFLHG

YLDDGPPFVCSSETLAKEIFSDARLELKLELTSPVHMKMDVFSVKLEPQQKTVLPEPTKE YLDDGPPFVCSSDTLAKEIFSDARLELKLELTSPVHMKMDVFSVKLEPQQKTVLPEPTKE YLDDGPPFVCSSETLEKEIFSDARLELKLELTSPVHMKMDVFSVKLEPQQKTVLPEPTKE YLDGGPPFVCSSETLAKEIFSDTRLELKLELTSPVHMKMDVFSVKLEPQQKTVLPEPTKE YLDDGPPFVCSSETLAKEIFSDARLELKLELTSPVHMKMDVFSVKLEPQQKTVLPEPTKE

Fig. 1.12: Alignment of the amino acid sequences of the ENU-3 protein family

The Clustal Omega alignment was manually manipulated to show the alignment of the

\section{last 13 identical amino acids.}




\subsection{Nuclear envelope and traffic mechanisms throughout the cell}

A putative classic bipartite nuclear localization sequence (Cooper, 2000) is found in the amino acid sequence of ENU-3 which consists of basic lysine $(\mathrm{K})$ or arginine $(\mathrm{R})$ residues interrupted by a series of other residues. Namely, in the protein sequence of ENU-3, there are two KK amino acids (residues 137-138) followed by 12 other residues, followed by the RKR sequence (residues 151-153). Similar sequences are found in all the ENU-3 paralogs except for K01G5.3. The nuclear localization sequence, in addition to the predicted trans-membrane domain, led to an analysis of these domains by observing the sub-cellular localization in a mammalian cell model.

\subsubsection{Structure of the nuclear envelope}

The nuclear envelope provides organization for the nuclear structure. It is a complex structure consisting of an outer nuclear membrane (ONM), continuous with the ER, and an inner nuclear membrane (INM), carrying nucleus-specific proteins, separated by the perinuclear luminal space (Gerace et al., 1978) (Figure 1.13). The membranes are barriers between cytoplasmic and nucleic contents and thus provide a compartmentalization of numerous distinct metabolic processes between the two biochemical compartments. Immediately underneath the INM are the nuclear lamina, which are polymers of the lamin intermediate filament proteins in addition to other trans-membrane resident proteins (Gerace et al., 1978). The meshwork of nuclear lamina functions to maintain the shape of the nucleus. In addition, the mechanical strength of the nucleus is attributed to the structural scaffold provided by the nuclear lamina (Gerace et al., 1978). Moreover, recent studies have shown that lamin is involved in regulation of gene expression as well as tethering the cytoplasmic skeleton and chromatin in close proximity to the nuclear 
envelope (Gruenbaum et al., 2005). There are four categories of lamin proteins found in mammals. Lamins A and C are expressed after differentiation and are encoded by one gene with alternative splice sites. In contrast, lamins B1 and B2 typically express in somatic cells during development and are encoded by two distinct genes (Stuurman, Heins \& Aebi, 1998).

Similar to plasma membranes, the nuclear membranes are phospholipid bilayers permeable to small, non-polar molecules. In the case of small, polar or large macromolecules, selective traffic in and out of the nucleus is mediated by 120 -nm nuclear pore complexes (NPCs) embedded in the nuclear membranes (Cooper, 2000). For instance, RNA and ribosomal subunits which are synthesized in the nucleus must be transported to the cytoplasm via NPCs where they function in protein translation. Alternatively, transcriptions factors, polymerases and other proteins required for nuclear specific processes such as replication and transcription must be transported from their site of synthesis, the cytosol, to the nucleus to ensure proper functioning. The composition of the nucleus is thus dictated by the regulated transport through the ONM and the INM via nuclear pore complexes (Cooper, 2000). 


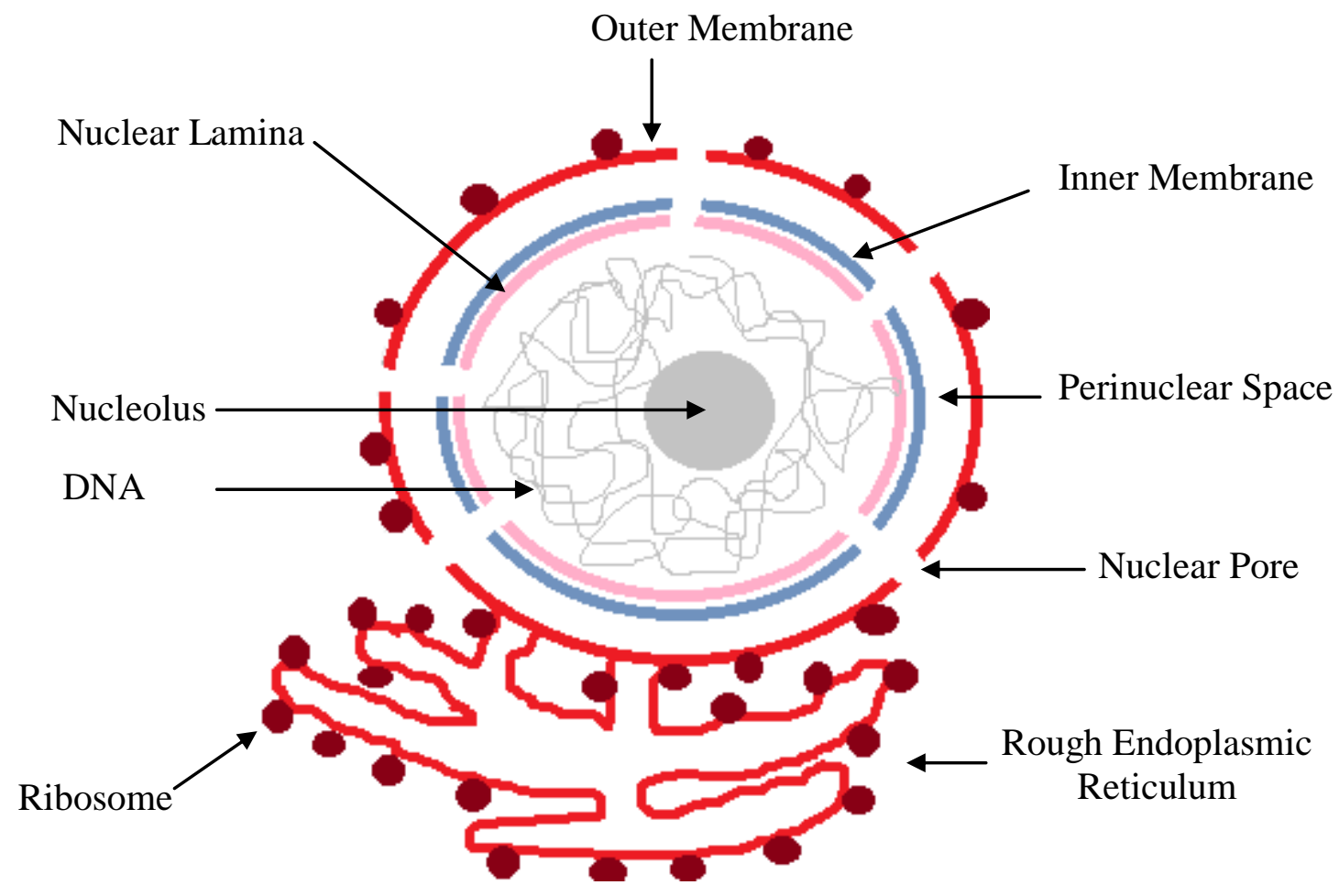

Figure 1.13: Nuclear envelope structure

The nuclear envelope encompasses the nucleus, consisting of the nucleolus and the genome, and is composed of an outer nuclear membrane and inner nuclear membrane separated by the perinuclear space. The meshwork of nuclear lamina lines the inner nuclear membrane and provides a structural scaffold for the nucleus. The outer nuclear membrane is continuous with the rough endoplasmic reticulum and thus contains ribosomes facing the cytosol. Transport across the nuclear membrane is mediated by nuclear pore complexes spanning the inner and outer nuclear membranes. 


\subsubsection{Resident proteins of the INM and ONM}

There have been over 60 trans-membrane proteins identified to reside in the INM and ONM with diverse complex functions, including chromatin regulation (Wilson \& Berk, 2010). The lamin binding receptor (LBR) protein found in the INM is involved in heterochromatin formation by binding to heterochromatin protein 1 (HP1) (Ye \& Worman, 1996). In addition, the LEM domain consisting of the mammalian INM

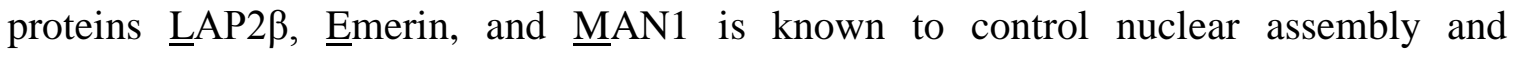
chromosome organization by directly interacting with BAF, a DNA-binding protein in the nucleus (Gruenbaum et al., 2005). Furthermore, in C. elegans, the INM protein UNC84, which interacts with lamin and the ONM protein ANC-1, which binds actin, have been linked to anchoring the actin cytoskeleton to the nuclear envelope, thus impacting cytoskeletal architecture. Similarly, the mammalian homolog of UNC-84, Sun-1, has been proven to interact in the perinuclear space with the ANC-1 mammalian homolog, nesprin 2 giant, responsible for binding actin (Worman \& Gundersen, 2006). Although most membrane proteins reside in the INM, several ONM proteins have been identified including nesprin family proteins homologous to Zyg-12 and UNC-83 in C.elegans, which interact with cytoskeleton components to regulate cytoskeletal arrangement of the cell (Worman \& Gundersen, 2006). The localization of these proteins in the ONM depend on tethering by the INM, Sun-1/UNC-84 proteins which can detect the C-terminal KASH domain (Klarsicht, Anc-1, Syne homology) common to all ONM proteins (Worman \& Gundersen, 2006). 


\subsubsection{Traffic into the nucleus via NPCs}

Proteins with nuclear localization signals migrate to the nucleus and bind to the nuclear pore complexes without requiring energy, thus initially do not pass through the pore. This interaction is mediated by the cytosolic receptor, importin which recognizes the nuclear localization sequence and directs its importin $\alpha$ subunit to bind to the sequence rich in basic lysine or arginine residues on the target protein, while the importin $\beta$ subunit binds to the cytoplasmic structures of the NPC, minimizing the gap between the target protein and the nuclear envelope (Cooper, 2000). Translocation across the nuclear membrane via the NPC necessitates energy using GTP hydrolysis and is mediated by the GTP-binding Ran protein. On the cytosolic side of the nucleus, there are enzymes that promote GTP hydrolysis, while on the nuclear side of the nuclear envelope, there are enzymes that promote GTP binding to Ran (Cooper, 2000). Thus the gradient of Ran/GTP, formed by a high concentration of Ran/GTP in the nucleus and a high concentration of Ran/GDP in the cytosol drives the directionality of nuclear import. The complex formed between importin $\alpha, \beta$ and the target protein on the NPC in the presence of high concentrations of Ran/GDP is carried through the NPC to high concentrations of Ran/GTP. Ran/GTP binds to importin $\beta$ causing the complex to dissociate, causing the target protein and importin $\alpha$ to be released (Cooper, 2000). The complex between Ran/GTP and importin $\beta$ is transported back to the cytoplasm where hydrolysis of GTP takes place allowing importin $\beta$ to take part in another round of nuclear import (Cooper, 2000). Resident proteins of the nuclear envelope are synthesized in the ER, similar to trans-membrane protein synthesis. The extracellular protein, importin directs its $\beta$ domain to bind to the nuclear localization amino acid sequence of the newly synthesized protein 
while its $\alpha$ domain binds to the cytosolic region of the NPC. This closes the gap between the protein and NPC allowing the nuclear membrane resident proteins to embed into the nuclear membrane via entry into the NPCs (Cooper, 2000).

\subsubsection{Protein trafficking throughout the cell}

Eukaryotic cells are highly organized, including division into many different membrane-enclosed, specialized compartments (Costaguta \& Payne, 2009). These different compartments are highly dynamic, with exchange of lipids and proteins through vesicular and tubular transport carriers between them. The secretory/endocytic membrane transport pathway involves traffic throughout the cell via vesicles containing the proteins of interest which bud off the donor organelle and fuse with the target organelle. Material entering the cell can be internalized across the plasma membrane and transported first to early endosomes where sorting of the material occurs. At this point it can be recycled back to the plasma membrane, transported to late endosomes followed by lysosomes for degradation or to the Golgi apparatus for more specialized sorting (Costaguta \& Payne, 2009) (Figure 1.14).

Transport of membrane proteins to the cell surface occurs through a related membrane traffic mechanism that similarly utilizes vesicular carriers. Following transcription of target proteins in the nucleus, the mature mRNA is transported to the cytoplasm for translation to occur on ribosomes lining the surface of the rough endoplasmic reticulum (ER). After protein production occurs via translocation into the $\mathrm{ER}$, initiated by the possession of a signal peptide at the N-terminus of the proteins, the synthesized proteins are transported to the Golgi apparatus via vesicles which bud off the ER (Connerly, 2010). They enter the cis side of the Golgi facing the ER and bud off 
through the trans side of the Golgi, facing the plasma membrane (Connerly, 2010). The target proteins move through Golgi cisternae while undergoing post-translational modifications. They are then packaged into vesicles which are directed back towards various compartments of the cells depending on the function of the proteins (Figure 1.14). The proper localization of these synthesized proteins contributes to the functionality of the cell and cell growth.

In contrast, certain proteins enter the secretory pathway if they possess a signal peptide sequence at the amino terminal (Greenfield \& High, 1999). This sequence functions to hinder translation of the protein by interacting with other cellular components, and ultimately targets it to the ER where the nascent protein is cotranslationally translocated into the ER via a translocon of which the Sec-61 complex is the major component (Greenfield \& High, 1999). The cytosolic signal recognition particle (SRP) complex detects and binds proteins with signal peptides (Greenfield \& High, 1999). The newly formed complex of the ribosome, target protein and SRP then docks on the SRP receptor expressed on the surface of the ER bringing the target protein in close vicinity to the heteromeric Sec-61 complex composed of $\alpha, \beta$, and $\gamma$ subunits (Greenfield \& High, 1999). If sorting of the protein to more specialized subcellular compartments such as secretory granules and lysosomes is required, then the protein is packaged in vesicles and transported to the Golgi (Greenfield \& High, 1999). The final stage of the secretory pathway involves the fusion of the secretory granules with the plasma membrane where the contents are released out of the cell via interactions between the vSNARE Snc2p of the vesicle and tSNARE Sso1p of the plasma membrane (Munson \& Hughson, 2002) (Figure 1.14). 


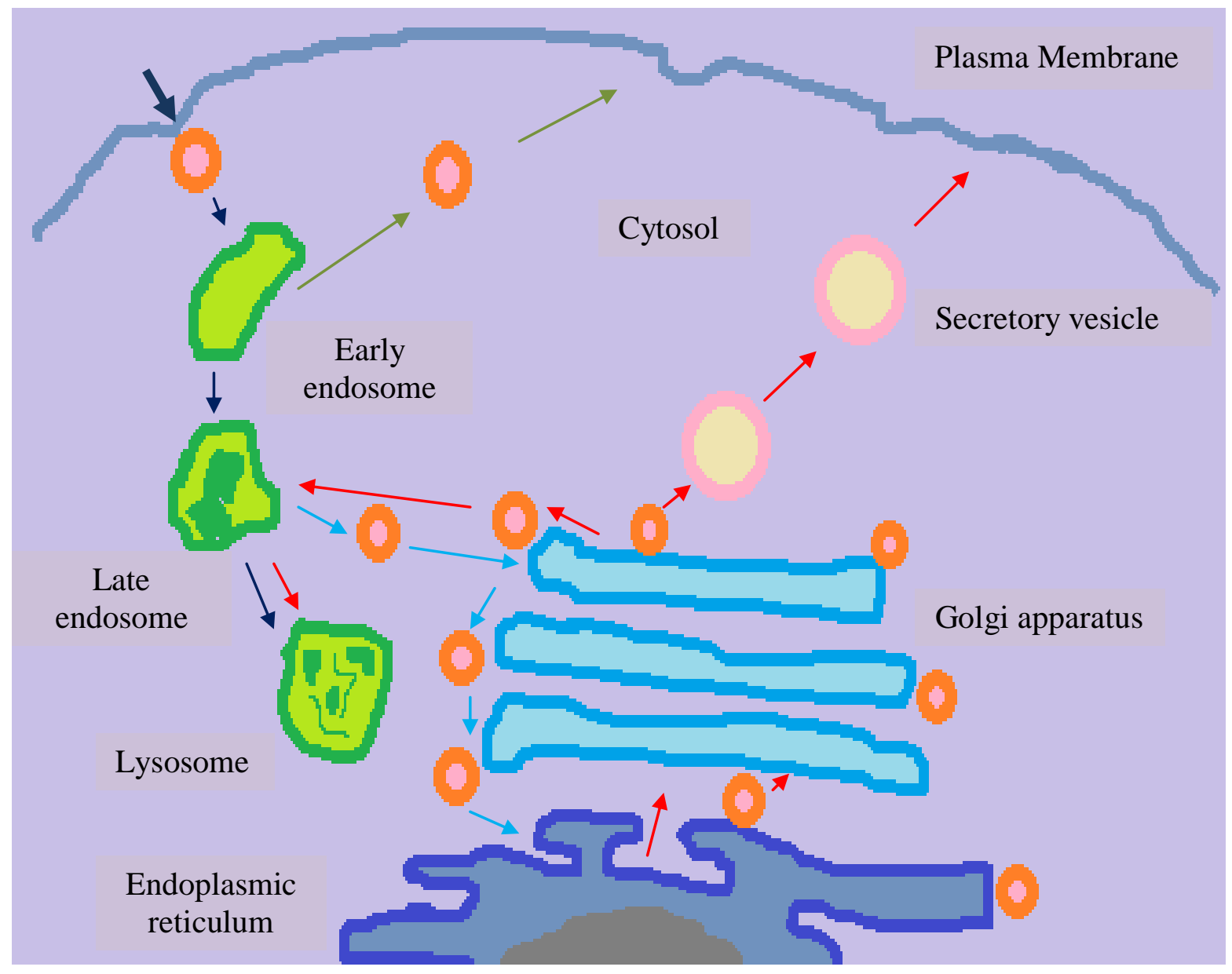

Figure 1.14: Traffic pathways throughout the cell

During endocytosis, protein cargo is internalized by the plasma membrane and transported to early endosomes and late endosomes for further sorting to the Golgi or to the lysosomes for degradation. In contrast, nascent proteins translocated via the Sec-61 complex and synthesized in the ER are transported to the Golgi via vesicular structures for further sorting, either to the plasma membrane for proteins destined for secretion or in the case of resident proteins, back to their respective sub-cellular compartments (Adapted from Connerly, 2010). 


\subsection{Hypothesis and objectives of thesis}

Previous work done with ENU-3 has shown that it is a protein involved in the nervous system as it expressed pan-neuronally in C. elegans, and two mutations in the enu-3 gene as well as knock-down of the mRNA by RNA inhibition enhanced DA and DB classes of motor neuron outgrowth defects in unc-5(e53) putative null mutants (Yee et al., 2011). Based on previous observations, my hypothesis that ENU-3 is a trans-membrane protein which functions in a pathway downstream of UNC-6/Netrin and parallel to UNC40/DCC to enhance mechanosensory neuron migration defects was tested. Thus, the first objective will focus on determining the genetic mechanism of ENU-3 through which it affects the guidance of mechanosensory neurons. Five additional paralogs of ENU-3 were identified all of unknown function and with a high similarity in amino acid sequence among each other. This led to my second thesis objective which included further investigating the roles and mechanism of all members of the ENU-3 protein family in enhancing motor neuron outgrowth defects. One hypothesis here is that the ENU-3 proteins function redundantly in motor neuron axon outgrowth so that knockdown of each one on its own should enhance the axon outgrowth defects of a strain lacking UNC5. Finally, the fact that ENU-3 and four of its paralogs contain a nuclear-localization sequence as well as a predicted trans-membrane domain, their expression patterns in mammalian HeLa cells and the significance of the two domains were the focus of my third objective. The hypothesis is that the ENU-3 family members with the transmembrane domains should be membrane proteins and the paralog without a predicted transmembrane domain should be in a distinct subcellular compartment. An additional aim of the project was to establish the expression patterns of the ENU-3 family 
proteins. All in all, it aimed to further the current understanding of the roles of ENU-3 and its paralogs in the development of the nervous system in C.elegans, and it aimed to ultimately determine if there are any homologous proteins involved in mammalian nervous system development. 


\section{Chapter 2. Materials and Methods}

\subsection{Handling of worm strains:}

Maintenance and handling of strains at $20^{\circ} \mathrm{C}$ were in accordance with previously described techniques (Brenner, 1974, Hope 1999). The wild type strain was represented by Bristol N2. Some mutants used in this study included: LG I: unc-40(e1430), unc40(tm5504), W03G9.3 (tm5189); LG III: enu-3(tm4519), C38D4.1 (tm5039); LG IV: unc-5(e53); LG V: him-5(e1420); X: unc-6(e78), unc-6(ev400) (WormBase, 2014). Strains were grown on standard NGM plates seeded with a lawn of the OP50 strain of $E$. coli and incubated at $16^{\circ} \mathrm{C}$ for medium term storage and frozen at $-80^{\circ} \mathrm{C}$ for long term storage.

\subsection{Genetic crosses:}

Construction of double mutant strains consisted of an initial parental cross between him-5(e1420);dpy20(e1282) males and hermaphrodites of one of the mutants of interest in a 5:1 ratio. F1 males were then crossed into hermaphrodites of the second mutant of interest. Eleven F2 heterozygote hermaphrodites were picked onto small, individual NGM plates. After self-fertilization, progeny were screened for the phenotypic traits of the parental mutants. In order to ensure homozygosis, the cross was continued in some cases for at least seven generations. Finally, mutations were confirmed by PCR using appropriate primers. Primers were designed to span the deletions of mutant strains, such as those including enu-3, while point mutations were further detected using restriction enzymes for strains consisting of unc-5(e53) and unc-40(e1430). The crosses were performed at $20^{\circ} \mathrm{C}$ under dissecting microscopes at 40x magnification, as well as under 
an epifluorescence dissection microscope (Leica, model MZFLIII) when screening for transgenic cross progeny was necessary.

\subsection{Transgenes}

Several transgenes were obtained from other studies and some were designed for use in this work in order to visualize individual motor and mechanosensory neurons as well as the expression pattern of several constructs.

\begin{tabular}{|c|c|c|c|}
\hline Array Name & Reporter Type & Expression & Reference \\
\hline$e v I s 82 b$ & $\begin{array}{l}\text { Transcriptional fusion } \\
\text { (unc-129p::gfp ::dpy20) } \\
\text { Integrated in the } \\
\text { genome }\end{array}$ & $\begin{array}{l}\text { Ventral nerve cord, DA } \\
\text { and DB motor neurons, } \\
\text { seam cells }\end{array}$ & $\begin{array}{l}\text { Colavita et al., } \\
1998\end{array}$ \\
\hline muIs32 & $\begin{array}{l}\text { Transcriptional fusion } \\
\text { (mec7p::gfp::dpy20) } \\
\text { Integrated in the } \\
\text { genome }\end{array}$ & $\begin{array}{l}\text { Mechanosensory } \\
\text { neurons }\end{array}$ & Ackley et al., 2001 \\
\hline $\operatorname{rqEx} 3 *$ & $\begin{array}{l}\text { Translational fusion } \\
\text { (enu-3p::enu-3::gfp) } \\
\text { extrachromosomal }\end{array}$ & $\begin{array}{l}\text { Ventral nerve cord, } \\
\text { motor neurons, } \\
\text { unidentified tail } \\
\text { neurons, PVT }\end{array}$ & $\begin{array}{l}\text { Yee, } 2011 \text { and this } \\
\text { study }\end{array}$ \\
\hline$r q E x 4$ & $\begin{array}{l}\text { Translational fusion } \\
\text { (mec-7p::enu-3::gfp) } \\
\text { extrachromosomal }\end{array}$ & $\begin{array}{l}\text { Mechanosensory } \\
\text { neurons }\end{array}$ & This study \\
\hline
\end{tabular}

Table 2.1: Integrated transgenes and constructed extrachromosomal arrays 


\subsection{Polymerase Chain Reaction (PCR)}

In order to confirm that the mutant strains constructed in this study contained the deletion within enu-3(tm4519), C38D4.1 (tm5039,) and W03G9.3 (tm5189), primers flanking the deletion region were designed and PCR was performed on the putative cross progeny. Point mutations of unc-40(e1430) and unc-5(e53) were detected with primers spanning a short region which included the single nucleotide changes. An additional step using restriction enzymes which recognized and cleaved adjacent to the nucleotide of interest generated appropriate sized bands in a DNA electrophoresis gel. In addition, gradient PCR was performed to amplify DNA for the reporter and rescue constructs as well as to amplify the cDNA of ENU-3, C38D4.1, K01G5.3, obtained from Thermo Scientific C.elegans cDNA ORF library and pcDNA3 mammalian expression vector for the mammalian cell expression analysis, supplied by Dr. Antonescu. All PCR steps were followed based on manufacturers' instructions (Thermo Scientific PCR Gradient Thermal Cycler) and as previously described (Garibyan \& Avashia, 2013). Annealing temperatures and extension times varied with the primers' annealing temperatures as well as the size of the expected amplicon (Refer to tables 6.3, 6.4, and 6.8 in appendices).

\subsection{Scoring of DA and DB motor neuron outgrowth defects}

DA and DB motor neuron cell bodies are located along the ventral cord sending their axons circumferentially to the dorsal side. The transgene evIs $82 b$, a transcriptional reporter gene where $2.5 \mathrm{~kb}$ of the neuronally expressed version of the unc-129 promoter is driving GFP is expressed in most of the DA and DB motor neuron cell bodies and axons but not the dorsal muscle band (Colavita et al., 1998). This allowed for the visualization and scoring of the DA and DB motor neuron axons in live animals under a 
compound upright microscope (Leica, model DM5000X) at 1000x magnification under oil immersion (Yee et al., 2011). Animals were mounted for observation under the upright research grade microscope by immobilizing L4 animals with $10 \mathrm{mM}$ levamisole, on $2 \%$ agarose pads (Hope, 1999). Animals with DA and DB motor neuron axons that failed to leave the cell body were individually scored as having outgrowth defects.

\subsection{Scoring of AVM and PVM mechanosensory neuron guidance defects}

AVM and PVM mechanosensory neuron cell bodies are located in the middle of the animal and send their axons ventrally where they turn towards the anterior side of the animal along the VNC. The transcriptional reporter $m u I s 32$, contains the $m e c-7 p$ driving GFP in the mechanosensory neurons allowed the visualization of all six mechanosensory neurons, of which AVM and PVM were screened for migration defects. A mutant phenotype manifested itself through the inability of the axon to reach the ventral cord, scored as a guidance defect (Yee et al., 2011). The animals were treated in the same manner as when scoring motor neuron outgrowth defects (Hope, 1999). The most common guidance defects noted were AVM and PVM axons that migrated laterally towards the anterior side of the animal without first migrating ventrally.

\subsection{RNAi (RNA interference)}

RNAi clones were obtained from the Ahringer clone library and handled as previously described (Kamath et al., 2003, Yee et al., 2011). The preparation of RNAi specific plates required the addition of carbenicillin at $25 \mu \mathrm{g} / \mathrm{ml}$ and $1 \mathrm{mM} \mathrm{IPTG}$, necessary for dsRNA production, after performing the standard NGM protocol as previously described (Hope, 1999). The RNAi clones of interest were grown overnight in $5 \mathrm{~mL} \mathrm{LB}$ broth in a shaking (250 rpm) $37^{\circ} \mathrm{C}$ incubator. The next day, the clones were spread on pre-poured RNAi 
plates and allowed to grow overnight. Typically, 10-20 L4 hermaphrodites were transferred onto the RNAi plates and F1 progeny were plated 72 hours later onto a new set of RNAi plates. From the next, F2, generation, 100 L4 animals were picked and scored for outgrowth or guidance defects.

\subsection{Seamless cloning for mammalian cell expression}

The construction of clones for the mammalian cell expression analysis of ENU-3, and two of the paralogs, C38D4.1 and K01G5.3, was approached by following the seamless cloning protocol (Quinn, 2005; Antonescu, 2013). The cDNA of interest, the insert, was sub-cloned into pcDNA3, a mammalian expression vector by designing specific primers. The forward primer required to amplify the insert was identical at the 5 , end to the sequence of the vector up to the insertion site, and the start of the insert sequence at the $3^{\prime}$ end of the primer. Its complement represented one of the two primers required for vector amplification. The second primer required for vector amplification was identical at the 5 'end to the sequence of the insert up to its end and the sequence downstream of the insertion into the vector sequence at the 3 ' end of the primer. Its compliment depicted the second primer needed to amplify the insert. The PCR program followed a typical protocol (Table 6.3 in Appendices), with the addition of a gradient annealing temperature of 40$64^{\circ} \mathrm{C}$ followed by a 12 minute elongation step for 18 cycles to find the optimum annealing conditions. In order to remove the original PCR templates by digesting the starting DNA which was methylated, 1:1 molar ratio of insert and vector was incubated with $1 \mu 1 \mathrm{DpnI}$ for 3 hours at $37^{\circ} \mathrm{C}$. Furthermore, this DNA mixture solution was then transformed into DH5- $\alpha$ competent cells as described by manufacturer's instructions (Invitrogen DH5- $\alpha$ competent cells, kindly provided by Dr. Antonescu and Dr. 
Fillingham's Lab, Ryerson University), grown overnight and clones were cultured in LB broth with the appropriate antibiotic. Plasmids were prepared using Sigma Aldrich midi kits (Sigma Aldrich, Carlsbad, CA). In order to insert a FLAG tag into the ENU-3 protein immediately after the signal peptide, an additional seamless cloning step was performed as previously described. The FLAG tag was inserted into ENU-3 and C38D4.1 after the signal peptide and after the initial predicted coiled-coil region of K01G5.3. To generate ENU-3::GFP another seamless cloning reaction was also used to insert GFP at the Cterminus of ENU-3 right before the stop codon. The identification of successful cloning of the insert into pcDNA3 was verified through DNA sequencing at the Peter Gilgan Centre for Research and Learning, funded by Dr. Antonescu.

\subsection{Cell culture}

HeLa (Henrietta Lacks) cells, derived from a cervical cancer tumor, were cultured in Dulbeccos's modified Eagle's medium (DMEM) (with glucose and glutamine; CellGro, Herndon, VA) with 10\% fetal bovine serum (FBS; Life Technologies, Inc. Carlsbad, Ca) and supplemented with 100 I.U./ml of penicillin and $100 \mathrm{ug} / \mathrm{ml}$ of streptomycin (ATCC, Inc. Carlsbad, Ca). All cells were grown at $37^{\circ} \mathrm{C}$ with $5 \% \mathrm{CO}_{2}$.

\subsection{Transfection and immunofluorescence staining of HeLa cells}

HeLa cells were plated at 20-30\% confluency on six-well plates one day prior to transfection. The manufacturer's protocol was followed for transfections with FuGENE ${ }^{\circledR}$ HD Transfection Reagent (Promega, Madison, WI). The plasmids containing the cDNA ORF of interest recombined in the pcDNA3 mammalian expression vector were diluted in sterile double distilled water at $1 \mu \mathrm{g} / \mathrm{well}$. For the co-transfection of FLAG::ENU-3 and ER marker, KDEL::GFP (Nasu-Nishimura et al., 2006), ( a kind gift from Dr. Greg 
Feirn's Lab, St. Michael's Hospital), $1 \mu \mathrm{g}$ of each DNA were mixed per well. For transfection, FuGENE ${ }^{\circledR}$ HD was added to the dilution mix and pipetted up and down 20 times to ensure complete mixing. After a 20 minute incubation period at room temperature, $44 \mu \mathrm{l}$ of the diluted plasmid mix was added dropwise to each well containing $0.8 \mathrm{~mL}$ of DMEM media supplemented with FBS and penicillin/streptomycin. After four hours, cells were washed in 1x PBS (CellGro), the media was replaced and cells were incubated overnight at $37^{\circ} \mathrm{C}$ with $5 \% \mathrm{CO}_{2}$.

After 24 hours, the cells were fixed with 4\% Paraformaldehyde solution (PFA, Electron Microscopy Sciences, Hatfield, PA) for 30 minutes and quenched with $100 \mathrm{mM}$ glycine followed by a 10 minute permeabilization period with Triton $\mathrm{X}-100$ (Bioshop). Cells were then blocked with 3\% Bovine Serum Albumin (BSA, BioShop) for 20 minutes. Primary anti FLAG, mouse, monoclonal or anti GFP, rabbit, polyclonal (Cell Signalling Technology) antibody solutions were made by diluting 1/400 of antibody in $1 \%$ BSA. Coverslips were flipped and immersed in $200 \mu \mathrm{l} /$ well of antibody solution on parafilm. After a one hour incubation period in the dark, cells were washed with 1x PBS and the secondary antibody solution (1/400 dilution) was added, $750 \mu 1 /$ well. Cy3 and Alexa 488 secondary antibodies were purchased from Molecular Probes, Invitrogen. After an additional hour of incubation, cells were washed, stained with $1 \mu \mathrm{g} / \mathrm{mL}$ DAPI solution at 1/5000 dilution, and mounted with one drop of DAKO fluorescent mounting medium (DAKO North America Inc., Carpinteria, CA). 


\subsection{Microscopy}

All genetic crosses were performed under dissecting microscopes at 40x magnification, as well as under an epifluorescence dissection microscope (Leica, model MZFLIII) when screening for transgenic cross progeny was necessary. The visualization and scoring of the DA, DB, AVM and PVM axons in live animals under a compound upright microscope (Leica, model DM5000X) was performed at 1000x magnification under oil immersion (Yee et al., 2011). Animals were mounted by immobilizing L4 animals with 10mM levamisole, on 2\% agarose pads (Hope, 1999). Microinjection was performed on a Zeiss Axiovert 10 where hermaphrodites were mounted on $2 \%$ agarose pads containing a small drop of halocarbon oil.

\subsection{Cell-surface labelling of HeLa cells}

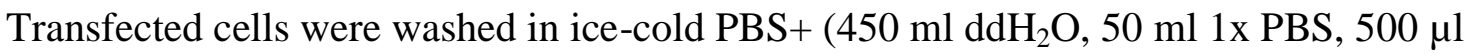
$\mathrm{CaCl}_{2}, 500 \mu 1 \mathrm{MgCl}_{2}$ ) and incubated with $3 \% \mathrm{BSA}$ for 15 minutes on ice. Primary antiFLAG, mouse, monoclonal or anti-GFP, rabbit, polyclonal was diluted 1/400 and added to the cells at $200 \mu \mathrm{l} /$ well. Cells were then fixed with $4 \%$ PFA for 30 minutes followed by additional 15 minute incubation in 3\% BSA. $750 \mu 1$ of Cy3 or Alexa 488 secondary antibody (Cell Signalling Technology) solution was diluted to $1 / 400$ and was added to each well, and incubated for 1 hour in the dark. Lastly, cells were stained with DAPI (1/5000 dilutions) and mounted with DAKO.

\subsection{Removal of ENU-3's predicted transmembrane domain}

ENU-3's predicted transmembrane domain (TM) was removed through site-directed mutagenesis. The forward primer was identical at the 5' end to the sequence of ENU-3 up to the putative transmembrane domain and identical to the sequence of ENU-3 
immediately after the predicted transmembrane domain at the 3' end of the primer. Its compliment represented the reverse primer. After a similar gradient PCR procedure was followed, (Table 6.3, Appendices), the amplicons were transformed into DH5- $\alpha$ competent cells, cultured overnight in LB broth and Ampicillin $(100 \mu \mathrm{g} / \mathrm{ml})$, followed by plasmid preparation using Sigma Aldrich midi prep kits. The clones were then sequenced for verification of the transmembrane domain deletion. The FLAG::ENU-3::GFP $(\Delta \mathrm{TM})$ construct was then transfected into HeLa cells at $1 \mu \mathrm{g} / \mathrm{well}$. After eight hours, the transfected cells were treated with Brefeldin A (Cell Signalling Technology) at $100 \mathrm{ng} / \mathrm{ml}$ for 12 hours and incubated at $37^{\circ} \mathrm{C}$ with $5 \% \mathrm{CO}_{2}$. The following day, cells were fixed, quenched with $100 \mathrm{mM}$ glycine and mounted with one drop of DAKO mounting medium.

\subsection{SDS-PAGE and Western blot}

The sizes of F::ENU-3::GFP and ENU-3::GFP were confirmed by running a 10\% SDS-PAGE gel as previously described (Mahmood, \& Yang, 2012) at $60 \mathrm{~V}$ for 30 minutes and $120 \mathrm{~V}$ for 90 minutes in 1x SDS-PAGE running buffer containing $100 \mathrm{ml}$ of

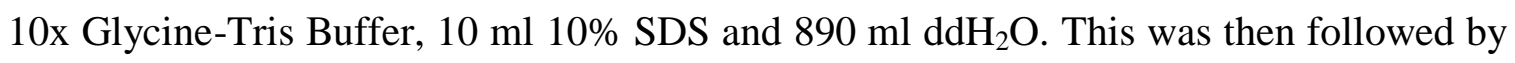
a two hour transfer step, as previously described (Mahmood, \& Yang, 2012), in Western Blotting transfer buffer containing $100 \mathrm{ml}$ of 10x Glycine-Tris buffer, 200ml methanol,

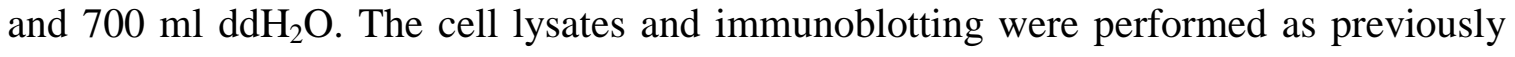
described (Antonescu et al., 2010) using a primary rabbit polyclonal anti-GFP antibody (Cell Signalling Technology) and a secondary goat polyclonal anti-rabbit antibody tagged with HRP (Cell Signalling Technology). The 1x immunoblot wash buffer contained 100 ml 10x Base immunoblot wash buffer ( $\mathrm{pH} 7.4$ ), $0.5 \mathrm{ml}$ Tween-20, and $0.5 \mathrm{ml} \mathrm{NP-40}$ alternative. 


\subsection{Construction of reporter constructs and microinjection of C. elegans}

As previously described, a PCR fusion protocol was followed to generate the translational fusion reporter constructs (Hobert, 2002, Yee et al., 2011). In order to confirm the expression pattern of ENU-3 in C.elegans, the H04D03.1 promoter region $(3 \mathrm{~kb})$ and gene $(1 \mathrm{~kb})$ were amplified. The forward primer was designed to bind $3 \mathrm{~kb}$ upstream of H04D03.1 gene and the reverse fusion primer bound to the sequence immediately before the stop codon of H04D03.1. LongAmp Taq Polymerase (New England Biolabs) was used to amplify the gene. This was then fused to a $1.8 \mathrm{~kb}$ fragment encoding GFP followed by the 1kb unc-54 3'UTR amplified from pD95.75 using TaKaRa Ex Taq Polymerase (TaKaRa Biotech Inc.). Following confirmation of correct sizes by PCR, the translational fusion construct fused with the GFP reporter gene was microinjected into $\mathrm{N} 2$ animals $(30 \mathrm{ng} / \mu \mathrm{l})$ with the co-injection marker myo-2::yfp (70 ng/ $\mu 1)$. This extrachromosomal array was entitled rqEx3 (Yee, 2011).

In order to address whether ENU-3 functions ventrally or dorsally to enhance mechanosensory neuron migration defects in $u n c-40$ mutants, an additional translational fusion fragment was constructed to quantify its effects when overexpressed in mechanosensory neurons in $u n c-40$ mutants using the mec-7 promoter to drive GFP expression in the mechanosensory neurons. Primers were designed to amplify only the $1 \mathrm{~kb}$ region of the H04D03.1 gene and then fused to the $1.8 \mathrm{~kb}$ fragment encoding GFP along with the $1 \mathrm{~kb}$ unc-54 3'UTR amplified from pD95.75 using TaKaRa Ex Taq Polymerase (TaKaRa Biotech Inc.) Additionally, the 800 bp mec-7 promoter was

amplified from p1525 which contains the mec-7 promoter driving unc-5, generously provided by Dr. Joe Culotti's Lab at Mt. Sinai Hospital. A final PCR reaction was 
performed to fuse the mec-7p amplicon to the H04D03.1 gene fused with GFP using TaKaRa Ex Taq Polymerase (TaKaRa Biotech Inc.). The resulting construct was microinjected into $u n c-40$ mutants $(70 \mathrm{ng} / \mu \mathrm{l})$ with the co-injection marker myo-2::yfp (30 $\mathrm{ng} / \mu 1)$. This extrachromosomal array was entitled rqEx4.

Microinjection was performed as previously described (Berkowitz et al., 2008) at Samuel Lunenfeld Research Institute at Mt. Sinai Hospital in Toronto. L4 to young, nongravide hermaphrodites were mounted on $2 \%$ agarose pads containing a small drop of halocarbon oil. Microinjection was performed on a Zeiss Axiovert 10. Animals that survived microinjection with a DNA total concentration at $100 \mathrm{ng} / \mu \mathrm{l}$, were placed on small NGM plates, adding M9 as needed to remove residual oil. After one generation of self-fertilization, the F1 generation was monitored for transgenic animals.

\subsection{Statistical methods}

Analysis of statistical significance between treated and control animals in the RNAi experiments was calculated using chi-squared tests and Two-Sample multivariate t-tests on a $95 \%$ confidence level, with $\alpha=0.05$ or smaller level of significance. The TwoSample multivariate t-test was also performed to compare the difference in motor neuron outgrowth defects and mechanosensory neuron migration defects between all strains constructed. ${ }^{\mathrm{a}}$ indicates a $\mathrm{p}$ value less than $0.05,{ }^{\mathrm{b}}$ indicates a $\mathrm{p}$ value of less than 0.01 , and ${ }^{c}$ indicates a p value of less than 0.001. The Statistical Analysis Software (SAS) was utilized for all statistical analyses (SAS, 2013). 
Chapter 3. Results

\section{1 enu-3 functions in an UNC-6/Netrin dependent pathway parallel to UNC- 40/DCC/Frazzled to enhance mechanosensory neurons guidance defects}

In order to investigate whether ENU-3 has an influence on the ventrally directed migrations of the AVM and PVM touch receptor neurons the final patterns were examined in live animals of various genetic backgrounds expressing the muIs32 transgene. Wild-type migration of AVMs and PVMs consists of the axons leaving the cell body located in the mid-body of the animal, making their way to the ventral cord where they turn and migrate towards the head of the animal (Fig 3.1 A). Abnormal trajectories are often seen with unc-40(e1430) mutant animals when the axons migrate straight across the midline of the animal failing to reach the ventral cord first, leading to an uncoordinated phenotype $($ Fig $3.1 \mathrm{~B})$. Without the UNC-40 receptor present on the developing growth cone, the chemoattraction response caused by sources of UNC-6 cannot be mediated. Often, the PVM cell body is misplaced to a more anterior position than in wild-type due to the fact that it does not migrate posteriorly from its initial anterior position, which also results in uncoordinated movement of animals (Chan et al., 1996). 

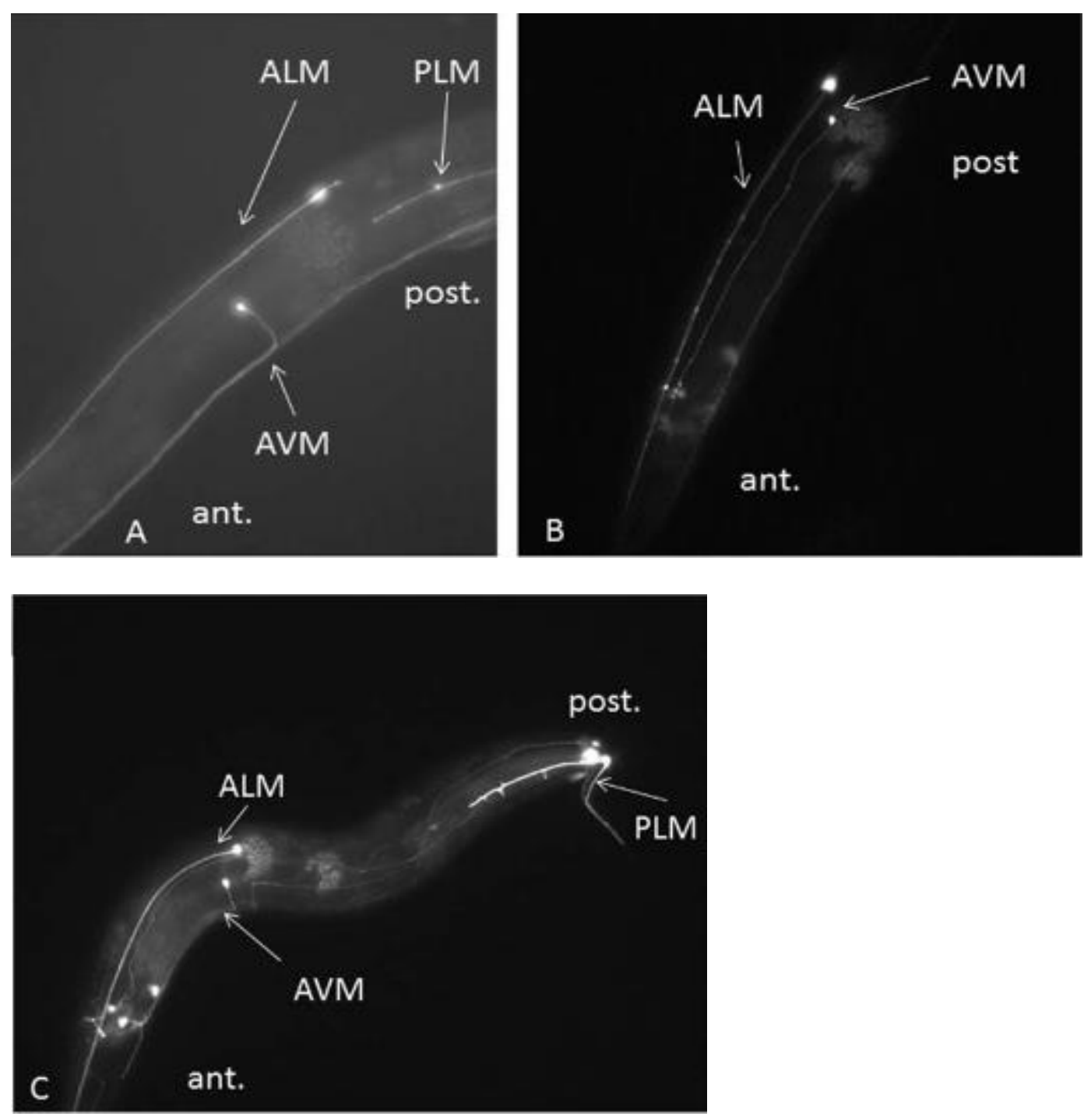

Figure 3.1: The mechanosensory neurons are affected by mutations in ENU-3 and UNC-40. A: AVM in a wild-type animal under x400 magnification. B: Defective AVM in an unc40(e1430) mutant at x200 magnification. C: Absent PVM and PLM defect in an unc40(e1430);enu-3(tm4519) mutant at x200 magnification (Yee et al., 2014). 
The UNC-6/Netrin pathway and the SLT-1 pathway are the only identified pathways, until recently, known to be responsible for migration of developing touch receptor neurons. The intricate balance between growth cones expressing UNC-40 receptors attracted towards a gradient of UNC-6 along the ventral cord and the repulsion of the same growth cones additionally expressing SAX-3 receptors by SLT-1 along the dorsal cord, dictates the wild-type migration pattern of developing axons. Moreover, in the absence of UNC-40 or UNC-6, the SLT-1 pathway tends to compensate for the Netrin pathway thus leading to increased signalling activity (Fujisawa et al., 2007).

In order to understand whether enu-3 functions in either the SLT-1 or the UNC-6 pathways, double mutants of enu-3(tm4519);unc-6(ev400) and enu-3(tm4519);slt-1(eh15) were scored for AVM and PVM migration defects. All animals contained the specific transgene responsible for visualization of the six mechanosensory neurons (muIS32) (Table 2.1). Table 3.1 indicates that there was a significant enhancement of guidance defects of AVM and PVM in enu-3(tm4519);slt-1(eh15) putative null double mutants compared to the slt-1(eh15) single mutant demonstrating that ENU-3 is in the parallel pathway to the SLT-1 pathway, most likely in the UNC-6 pathway (Yee et al., 2014). The fact that enu-3(tm4519);unc-6(ev400) double mutants did not appear to have significantly enhanced migration defects compared to the unc-6(ev400) putative null single mutant further strengthens the idea that ENU-3 is working in the UNC-6 pathway to affect migration of developing AVM and PVM touch receptor neurons. In order to demonstrate whether enu-3 does in fact function with the UNC-6/UNC-40 pathway; double mutants were constructed and scored for guidance defects. епи-3 alone does not appear to have any effects on the migration of AVM and PVM, however the double mutant unc- 
40(e1430);enu-3(tm4519) demonstrated significant enhancement of defects as compared to either of the single mutants, as shown in Table 3.1 and Figure 3.1 C (Yee et al., 2014). In addition, in the animal in Fig. 3.1C the PLM migration is abnormal as it may be detached from its normal attachment point. Most of the defective AVMs were migrating anteriorly towards the head of the animal, while PVM defects consisted of misplaced cell bodies, indicative of incomplete migration of PVM to the posterior side of the animal during development, or a mixture of sending the axon dorsally or anteriorly. These findings suggest that ENU-3 is working parallel to UNC-40 to provide an instructive role for AVM and PVM, in a pathway dependent on UNC-6 to enhance guidance defects. Additionally, Table 3.1 shows that guidance defects are less pronounced in the unc-40 mutant compared to the unc-6(ev400) mutant, the only known receptor for UNC-6. The fact that the double mutants lacking UNC-40 and ENU-3 have more defects than the unc40 mutant and are close to those found in the putative unc- 6 null mutant support the idea that ENU-3 itself may be a second receptor itself or in the pathway of another unknown receptor able to respond to UNC-6 and promote guidance defects. 


\begin{tabular}{lccc}
\multicolumn{1}{c}{ Strain } & \% AVM & $\%$ PVM & \\
\multicolumn{1}{c}{ (with mec7p::GFP) } & Defective & Defective & N \\
\hline enu-3(tm4519) & 1 & 0 & 100 \\
unc-40(e1430) & 14 & 24 & 100 \\
unc-40(e1430);enu-3(tm4519) & $33^{\mathrm{b}}$ & $42^{\mathrm{b}}$ & 105 \\
unc-6(ev400) & 36 & 46 & 100 \\
enu-3(tm4519); unc-6(ev400) & 37 & 47 & 105 \\
slt-1(eh15) & 40 & 3 & 109 \\
enu-3(tm4519);slt-1(eh15) & $53^{\mathrm{a}}$ & $7^{\mathrm{a}}$ & 120 \\
\hline
\end{tabular}

Table 3.1: Mechanosensory neurons migration defects are enhanced by enu-3.

The strains indicated were scored for AVM and PVM guidance defects defined as failure of axons to reach the ventral cord and misplacements or absence of cell bodies (Yee et al., 2014).

${ }^{a}$ indicates a $p$ value of less than 0.05

${ }^{\mathrm{b}}$ indicates a p value of less than 0.01 on a chi squared test. 
In order to support that enu-3 enhanced the guidance defects of AVM and PVM, RNA interference using clones from the Ahringer RNAi library (Fraser, 2000; Kamath et al., 2003) was performed in two unc-40 allele (e1430 and tm5504) strains as well as the enu-3(tm4519) strain. Figure 3.2 shows that when the enu-3 mRNA was reduced by RNAi, in both unc-40(e1430) and unc-40(tm5504), there was a significant enhancement of AVM defects compared to the mutants which received the empty L4440 vector (Yee et al., 2014). PVM defects were also enhanced when enu-3 mRNA was reduced, but reduction was only observed in unc-40(e1430) mutants. Furthermore, when unc-40 mRNA was reduced in wild-type and enu-3 mutants, an enhancement of migration defects was observed in the AVM for both strains, compared to animals which were only fed with empty vector. The enhancement of AVM defects was larger than in wild-type animals when unc-40 mRNA was reduced. Finally, PVM defects were also enhanced when unc-40 mRNA was reduced in enu-3 mutants but not in the wild-type strain. These observations further supported the idea that ENU-3 enhances touch receptor neurons guidance defects affecting AVMs to a greater degree than PVMs.

It is evident that both genetic data and RNAi data demonstrate an important role for ENU-3 working in parallel to UNC-40, dependent on UNC-6, to affect guidance of the AVM and PVM neurons, thorough a currently unknown mechanism (Yee et al., 2014). Finding a mutant that enhances the defects of UNC-40 in AVM and PVM is highly significant as most of the mutants found to date are either in the UNC-40 or the SLT1/Robo pathways. 


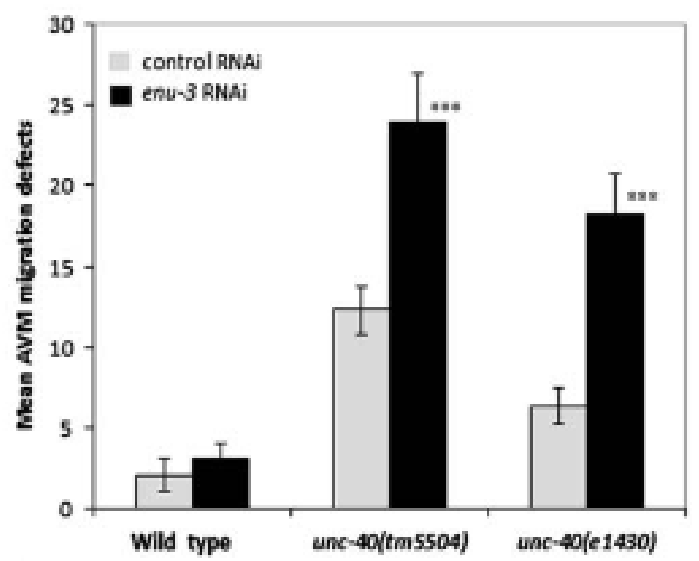

A

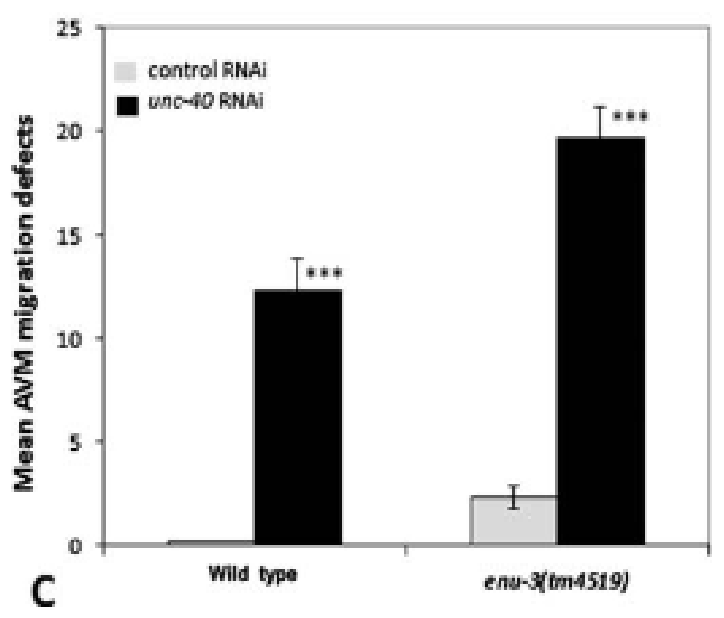

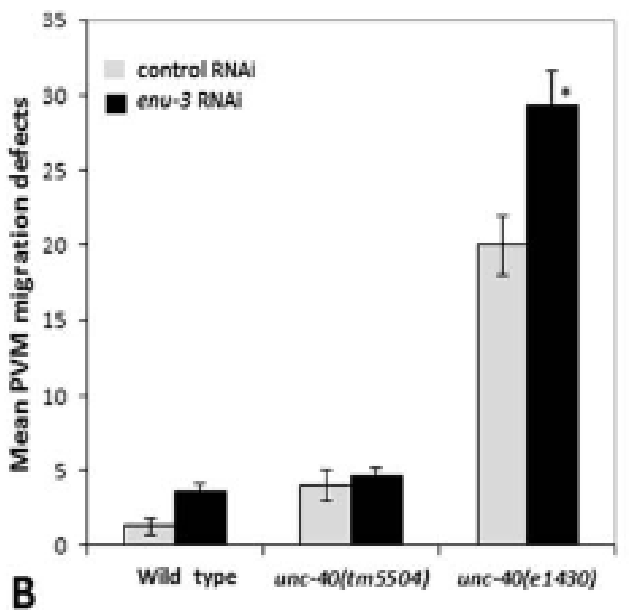

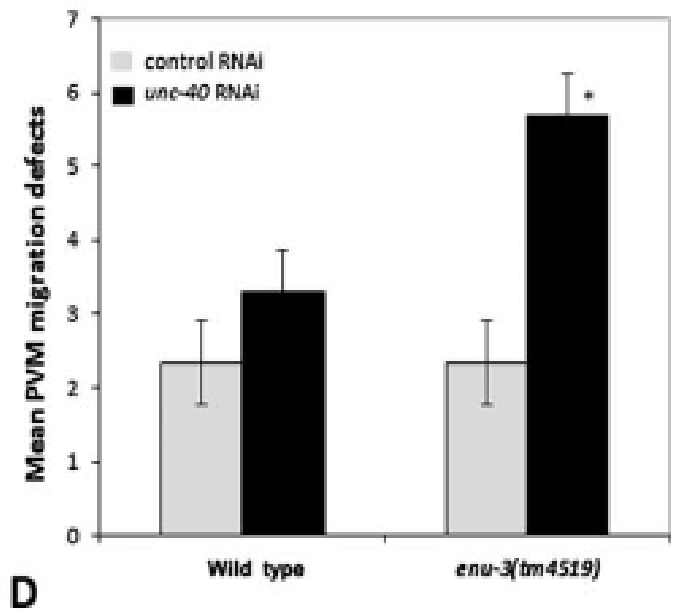

Figure 3.2: RNA interference knockdown of ENU-3 and UNC-40 levels enhance AVM and PVM guidance defects (Yee et al., 2014)

Each strain indicated was fed with bacteria containing the control empty plasmid, pL4440, or with plasmids containing an insert for the gene of interest (Kamath et al., 2013). Three separate trials were performed and their mean percentage of affected axons and standard deviation are plotted and depicted on the Y axis. Each trial consisted of 100 animals counted. A and B: AVM and PVM defects, respectively, of animals treated with control plasmid or the enu-3/H04D03.1 RNAi clone. C and D: AVM and PVM defects, respectively, of animals treated with control plasmid or the $u n c-40$ RNAi clone. ${ }^{*} p$ value less than $0.05,{ }^{* *} p$ value less than $0.01,{ }^{* * *} p$ value less than 0.001 on a chi squared test (Yee et al., 2014). 
Does ENU-3 work to guide the PVMs and AVMs towards the ventral cord as UNC40 does or does it normally oppose migration towards the ventral cord as UNC-5 does in the motor neurons? Genetic data and RNAi data indicate that ENU-3 is functioning to guide touch receptor neurons in the ventral direction, similar to UNC-40. A translational fusion PCR product was constructed and microinjected into L4 unc-40(1430) animals to address this issue. The construct, ENU-3::GFP driven by the strong mec-7p promoter was microinjected into unc-40(e1430) mutants in order to observe whether the microinjected progeny result in enhancement or suppression of AVM and PVM guidance defects. Knowing that UNC-40 functions ventrally, chemo-attracted by sources of UNC-6 along the ventral cord, unc-40 mutants tend to fail to send their axons ventrally, unlike in wildtype animals. Thus, an over-expression of ENU-3 in unc-40 mutants should suppress the guidance defects, if ENU-3 is working in the same ventral-ward direction as UNC-40.

Microinjection was attempted numerous times and it did not prove successful at the time. Consequently, two possible issues were identified, namely the concentration of ENU-3 microinjected $(70 \mathrm{ng} / \mu \mathrm{l})$ was too high and possibly toxic to the animals, or the presence of technical difficulties hindered the success of microinjection. Firstly, the concentration of ENU-3 was lowered at $50 \mathrm{ng} / \mu \mathrm{l}$ as well as $30 \mathrm{ng} / \mu \mathrm{l}$, and re-attempted to be microinjected into mutant animals. This strategy continued to prove unsuccessful as animals were usually dying immediately after microinjection or shortly after. This led to the second trouble shooting strategy, namely to investigate whether technical issues were involved. Thus, plasmids that have been previously used and successfully microinjected into animals such as rqEx 3 (Yee, 2011), or simply the co-injection marker myo-2::yfp, were microinjected into wild-type animals. This again proved unsuccessful after 
numerous attempts, leading to the conclusion that technical difficulties associated with handling the micro-injection apparatus did not allow for successful generation of transgenic animals. Future experiments will further investigate this issue and attempt to eventually determine the effects of the transgenic animals. 


\subsection{Knockdowns of the paralogs of ENU-3 do not enhance guidance defects of AVM and PVM mechanosensory neurons}

Given that there was strong support for enu-3's function in enhancing guidance defects of AVM and PVM, as well as the fact that paralogs of ENU-3 have a high similarity in amino acid structure, their role in touch receptor neuron guidance was also investigated. This was performed in a similar fashion to ENU-3 by utilizing RNAi knockdown to reduce mRNA of each paralog independently in a wild-type strain as well as the two unc-40 allele strains aforementioned. Table 3.2 omits the data showing the knockdown of the paralogs in a wild-type strain as each paralog on its own does not cause any defects, similar to enu-3. It is evident that when enu-3 mRNA levels were reduced in both unc-40(e1430) and unc-40(tm5504) strains, there was a statistically significant enhancement of AVM defects in these mutants compared to the mutants given the treatment of the empty vector L4440. On the other hand, mRNA reduction of the five paralogs did not seem to enhance AVM defects in either of the unc-40 mutants, compared to strains treated with the empty L4440 vector. PVM defects were also enhanced but to a lesser degree than AVM when enu-3 mRNA was reduced in $u n c-40$ mutants as shown in Table 3.2. Additionally, the reduction of the paralogs' mRNA did not affect the PVM guidance defects. These findings reduced the possibility of any involvement of the paralogs of ENU-3 in AVM and PVM axon guidance. 


\begin{tabular}{|c|c|c|c|c|}
\hline $\begin{array}{l}\text { Strain } \\
(m e c-7:: g f p)\end{array}$ & treatment & $\% \mathrm{AVM}$ & $\%$ PVM & $n$ \\
\hline \multirow{7}{*}{$u n c-40(t m 5504)$} & L4440 & 11.3 & 4 & 300 \\
\hline & H04D03.1 & $23.6^{a}$ & 6 & 300 \\
\hline & W0.5F2.2 & 11.3 & 4.3 & 300 \\
\hline & K01G5.3 & 11.6 & 1.33 & 300 \\
\hline & C38D4.1 & 12.6 & 2.33 & 300 \\
\hline & Y37D8A. 12 & 14.6 & 2.6 & 300 \\
\hline & W03G9.3 & 12.3 & 3 & 300 \\
\hline \multirow[t]{7}{*}{$u n c-40(e 1430)$} & L4440 & 9.66 & 29.3 & 300 \\
\hline & H04D03.1 & $23.6^{a}$ & 45 & 300 \\
\hline & W05F2.2 & 11.3 & 29.3 & 300 \\
\hline & K01G5.3 & 11 & 28 & 300 \\
\hline & C38D4.1 & 8.66 & 28.6 & 300 \\
\hline & Y37D8A. 12 & 9.33 & 27 & 300 \\
\hline & W03G9.3 & 11.3 & 26.6 & 300 \\
\hline
\end{tabular}

Table 3.2: RNAi knockdown of the ENU-3 paralogs did not enhance guidance defects of mechanosensory neurons

Each strain indicated was treated with bacteria containing either the control empty vector L4440 or vectors containing the insert for each of the six paralogs independently. Three trials were performed, 100 animals per trial, and their mean \% AVM and \% PVM defects are shown.

${ }^{a}$ indicates a $\mathrm{p}$ value of less than 0.05 on a chi-squared test. 


\subsection{ENU-3 enhances motor neuron outgrowth defects of unc-5 and unc-6 mutants}

The data indicating that ENU-3 appeared to function in the UNC-6/Netrin pathway enhancing guidance defects of mechanosensory neurons led to the investigation of potential roles ENU-3 may have in affecting motor neuron outgrowth defects. After constructing the strains of interest, it was found that unc-5(e53) mutants had a relatively low penetrance of outgrowth defects, defined as motor neuron axons failing to leave the cell bodies located along the ventral nerve cord, while enu-3 single mutants had negligible outgrowth defects. Interestingly, еnu-3 enhanced the outgrowth defects of unc5 mutants, with approximately double the amount of outgrowth defects found in the double compared to the unc-5(e53) single mutant, as show in Table 3.3.

In addition, enu-3(tm4519);unc-6(ev400) mutants had increased outgrowth defects compared to the single null unc-6(ev400) mutant suggesting that in the case of the motor neurons ENU-3 functions parallel to UNC-6 and UNC-5. The DB subset of motor neurons, especially, DB4, DB5 and DB6 were the most affected neurons when counting their outgrowth defects. Furthermore, unc-40(e1430);enu-3(tm4519) double mutants did not appear to have any outgrowth defects, as well as the individual single mutants. Lastly, the triple mutant unc-40(e1430);enu-3(tm4519);unc-6(e78) had enhanced outgrowth defects when compared to the unc-40(e1430));unc-6(e78) mutant (Table 3.3). These findings suggest that ENU-3 is involved in a pathway parallel to that of UNC-6/Netrin, independent of the UNC-6 ligand or its receptors, UNC-40 and UNC-5, to enhance motor neuron outgrowth defects. 


\begin{tabular}{lccccccc}
\hline \multicolumn{1}{c}{$\begin{array}{c}\text { Strain } \\
\text { (with evIs82b) }\end{array}$} & DA3 & DB4 & DA4 & DB5 & DA5 & DB6 & DA6 \\
& & & & & & & \\
\hline enu-3(tm4519) & 0 & 1 & 0 & 1 & 0 & 0 & 0 \\
unc-5(e53) & 1 & 12 & 4 & 12 & 5 & 2 & 2 \\
enu-3(tm4519); unc-5(e53) & $8^{\mathrm{a}}$ & 13 & 4 & $24^{\mathrm{a}}$ & 7 & $12^{\mathrm{a}}$ & 5 \\
unc-40(e1430); unc-5(e53) & 0 & 4 & 1 & 3 & 0 & 1 & 0 \\
unc-40(e1430); & 2 & $12^{\mathrm{a}}$ & 0 & $14^{\mathrm{b}}$ & 1 & $7^{\mathrm{a}}$ & 0 \\
enu-3(tm4519); unc-5(e53) & & & & & & & \\
unc-6(ev400) & 1 & 2 & 1 & 17 & 1 & 3 & 0 \\
enu-3(tm4519); unc-6(ev400) & 4 & 6 & 4 & $32^{\mathrm{a}}$ & 1 & 5 & 1 \\
unc-6(e78) & 9 & 24 & 4 & 36 & 3 & 11 & 0 \\
enu-3(tm4519)unc-6(e78) & 3 & 22 & 4 & $55^{\mathrm{a}}$ & 1 & 14 & 2 \\
unc-40(e1430);enu-3(tm4519) & 0 & 1 & 0 & 1 & 1 & 0 & 0 \\
unc-40(e1430);unc-6(e78) & 0 & 1 & 0 & 1 & 0 & 0 & 0 \\
unc-40(e1430); & 0 & $16^{\mathrm{c}}$ & 2 & $24^{\mathrm{c}}$ & 4 & $6^{\mathrm{a}}$ & 0 \\
enu-3(tm4519); unc-6(e78) & & & & & & & \\
\hline & & & & & & & \\
\hline
\end{tabular}

Table 3.3: UNC-40 and enu-3 enhance motor neuron outgrowth defects

The DA and DB motor neuron axons which failed to leave the cell body along the ventral nerve cord were counted as defective, $\mathrm{n}=100$ for each strain indicated.

${ }^{\mathrm{a}}$ indicates a $\mathrm{p}$ value of less than 0.05

${ }^{\mathrm{b}}$ indicates a $\mathrm{p}$ value of less than 0.01

${ }^{\mathrm{c}}$ indicates a $\mathrm{p}$ value of less than 0.001 on a chi squared test. 


\subsection{Motor neuron outgrowth defects are partially dependent on the presence of UNC-40}

After scoring the outgrowth defects of the putative null mutant unc-6(ev400) and the hypomorph unc-6(e78), both enhanced by enu-3, it was found, unexpectedly, that the null mutant had a lower amount of outgrowth defects as opposed to the hypomorph mutant, as shown in Table 3.3. This led to the investigation of possible reasons behind this observation. It is plausible that the unc-6(e78) allele can interact with UNC-40, allowing the attraction between the UNC-6 ligand and UNC-40 receptor, and in turn causing more outgrowth defects than the unc-6(ev400) null allele. Thus, the double mutant unc40(e1430); unc-6(e78) was constructed to observe if the depletion of UNC-40 will cause a suppression of outgrowth defects. Table 3.3 showed that this double mutant had negligible outgrowth defects of motor neurons, supporting the idea that the presence of UNC-40 is partially responsible for motor neuron outgrowth defects (Yee et al., 2014). Additionally, the double mutant unc-40(e1430);unc-5(e53) had less outgrowth defects compared to the unc-5(e53) single mutant, further emphasizing that the presence of UNC-40 enhanced outgrowth defects of DB4, DB5 and DB6 motor neurons in particular (Yee et al., 2014).

Moreover, the genetic construction of an additional triple, unc-40(e1430);enu3(tm4519);unc-5(e53), was performed in order to further investigate the effect of the presence of UNC-40, and enu-3 on motor neuron outgrowth defects. Table 3.3 showed that this triple had more outgrowth defects than the unc-40(e1430);unc-5(e53) double mutant, however, the outgrowth defects were less than observed in the enu3(tm4519);unc-5(e53) double mutant. This further confirms the previously stated idea 
that ENU-3 is likely to function independently of UNC-40 and UNC-5 to cause enhanced outgrowth defects. Also, it was apparent that ENU-3 may also cause additional outgrowth defects that are dependent on the presence of UNC-40 (Yee et al., 2014).

\subsection{Protein structure of the ENU-3 family members}

The SMART database was used to predict the protein structure of the ENU-3 family members and their similarity to ENU-3 is shown in Figure 3.3 (Yee, 2011). All paralogs except for K01G5.3, are more than 90\% identical at the N-terminal to ENU-3 and all possess similar key features as ENU-3 with the exception of a longer intracellular region. All five paralogs thus contain a predicted signal peptide from amino acid 1 to amino acid 23, a trans-membrane domain from amino acid 108 to 128 , and coiled-coil regions. Additionally, the five paralogs contain a predicted bipartite nuclear localization signal, residues KK (amino acids 137-138), followed by 12 residues, and three basic residues RKR (amino acid 151-153). On the other hand, K01G5.3 is highly similar to the four paralogs but not to ENU-3, as shown in Figure 3.3. K01G5.3 is not predicted to contain a signal peptide, a trans-membrane domain or a nuclear localization signal.

Although there are homologous proteins of ENU-3 in other worm species such as $C$. briggsae and $C$. remanei, statistically significant homologous proteins in higher organisms have not been found, suggesting possible homologies at the structural level rather than at the amino acid sequence level. 


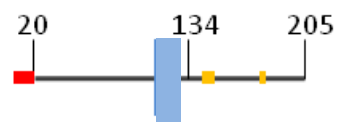

H04D03.1
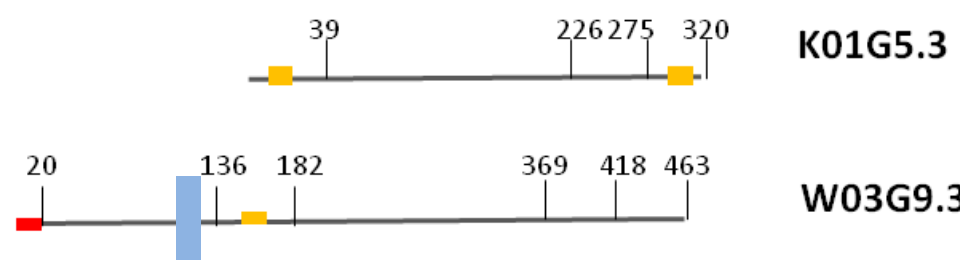

W03G9.3

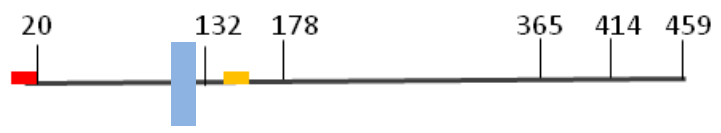

Y37D8A.12a

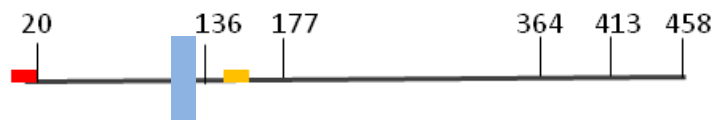

W05F2.2

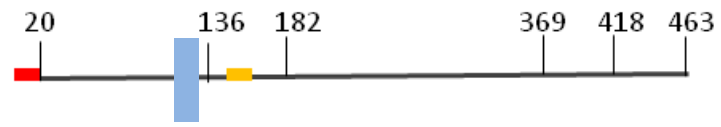

C38D4.1

Figure 3.3: Predicted structure of ENU-3 family members using SMART database.

The paralogs are highly similar to ENU-3 except for a longer intracellular region. K01G5.3 is the exception paralog that is highly identical to the rest of the paralogs at the C-terminal but not to ENU-3. The predicted signal peptide is shown in red, the transmembrane domain in blue, and the coiled-coil regions are represented in yellow after the STRING database representation. The homology at the amino acid level is shown in Figure 1.12 of the introduction 


\subsection{The ENU-3 protein family functions in a parallel pathway to the UNC-6/Netrin pathway to enhance outgrowth defects of motor neurons}

In order to further prove that ENU-3 had a role in affecting motor neuron outgrowth defects independent of the UNC-6 ligand, or the receptors UNC-40 and UNC5 receptor, RNAi knockdown was performed first in unc-5 mutants. In addition, since the paralogs of ENU-3 are highly identical in amino acid structure to ENU-3, these proteins were also knocked down by RNAi in unc-5 mutants to observe if they posses a role in motor neuron outgrowth, similar to ENU-3. Figure 3.4 demonstrates that when unc-5 mutants were fed with RNAi vectors containing the insert of interest, either enu-3 or each of the five paralogs, individually, there was an enhancement of outgrowth defects observed primarily in the DB4, DB5 and DB6 motor neurons compared to the animals that were fed with a control empty plasmid L4440. This trend further strengthened the genetic data supporting ENU-3's function in affecting motor neuron outgrowth independent of UNC-5. Additionally, this RNAi data indicated that all the paralogs enhance outgrowth defects in unc-5 mutants, similar to ENU-3. A similar RNAi knockdown was performed in enu-3(tm4519);unc-6(ev400) mutants where animals were fed with L4440 plasmids as control, or animals were fed with plasmids containing the RNAi clone of each of the other paralogs separately. This was performed in order to investigate whether the paralogs enhanced outgrowth defects, independent of UNC-6, similar to ENU-3. As expected, figure 3.5 demonstrated that the knockdown of each of the paralogs in the ENU-3 protein family did in fact enhance motor neuron outgrowth defects of enu-3(tm4519);unc-6(ev400) mutants with a particular effect on DB5. Furthermore, the RNAi knockdown of enu-3 and its paralogs independently in an unc- 
40(e1430);unc-5(e53) mutant background (Figure 3.6) generated a similar trend, that of an enhancement of outgrowth defects particularly of the DB4 and DB5 when compared to the animals treated with the control L4440 plasmid suggesting their possible UNC-40 and UNC-5 independent roles in affecting motor neuron outgrowth defects. Parallel experiments were conducted by Victoria Hipolito who knocked down several members of the ENU-3 family in an unc-5(e53), and in an enu-3(tm4519);unc-5(e53) background and found that knock-down of more than one ENU-3 family member increased the penetrance of motor axon outgrowth defects in DB4-DB6 predominantly but also in the other motor neuron axons (personal communication, Hipolito 2014). All in all, these RNAi findings strengthened the genetic knockout data of the double and triple mutants constructed, and suggested that ENU-3 and its five paralogs function redundantly in an UNC-6/Netrin independent pathway to enhance motor neuron outgrowth defects.

It is important to note that the three RNAi sets of knockdowns of the ENU-3 protein family members in unc-5(e53), enu-3(tm4519);unc-6(ev400) and unc-40(e1430);unc5(e53) backgrounds complimented the previously mentioned idea of an additional function of ENU-3, and its paralogs, dependent on the presence of UNC-40, where their absence was shown to cause more outgrowth defects. Although all three sets of RNAi knockdowns showed a similar trend of enhancement of outgrowth defects, the total number of defects were higher in the background strains containing functional UNC-40, namely unc-5(e53) and enu-3(tm4519);unc-6(ev400) backgrounds, than in the mutant background with a lack of UNC-40, namely unc-40(e1430);unc-5(e53). Thus, all members of the ENU-3 protein family seemed to cause additional motor neuron outgrowth defects dependent on the presence of UNC-40. 


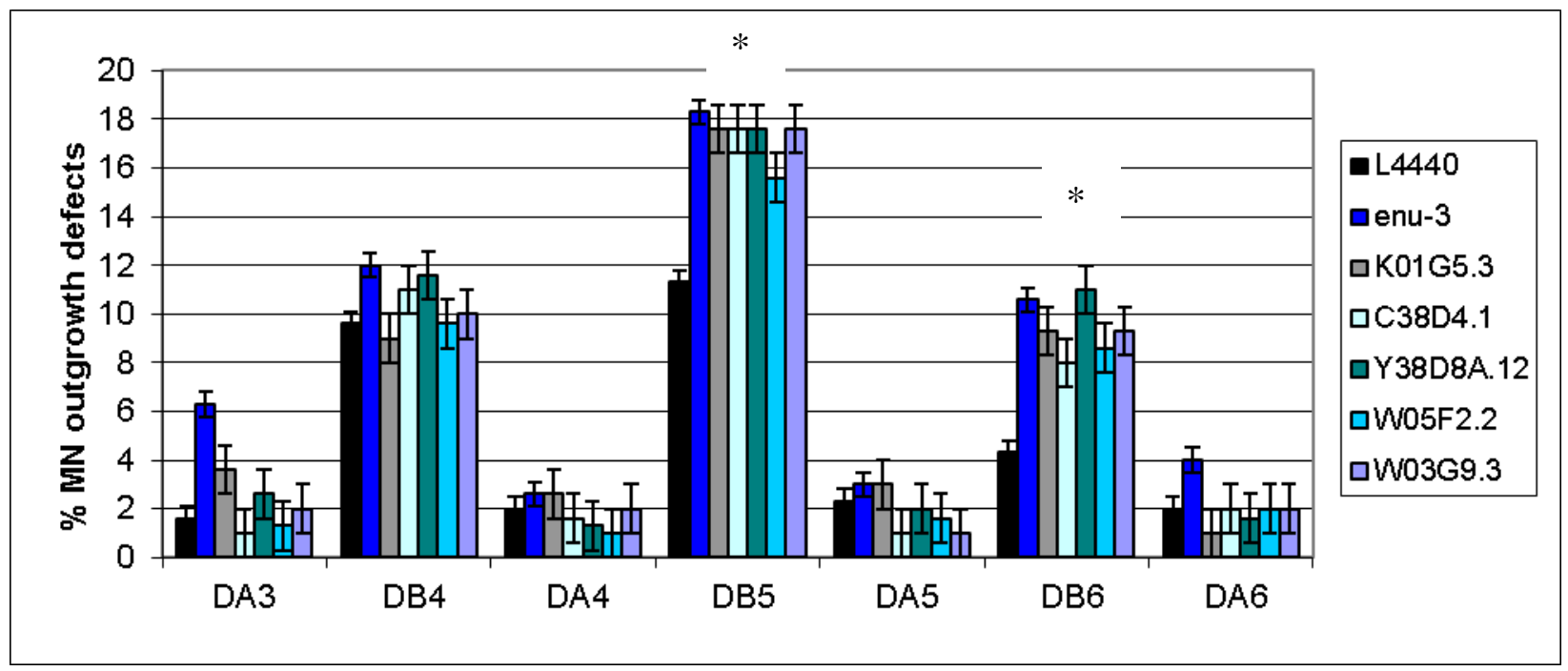

Figure 3.4: RNAi knockdown of the ENU-3 protein family enhances motor neuron outgrowth defects in $u n c-5(e 53)$ mutants.

unc-5(e53) mutants were treated with empty the RNAi vector L4440 or the vector containing the RNAi clone specified, and F2 progeny were scored for outgrowth defects. The average score from the three trials performed $(n=100 /$ trial $)$ and standard errors were plotted. $* p$ value less than $0.05, * * p$ value less than $0.01, * * * p$ value less than 0.001 on a chi squared test. 


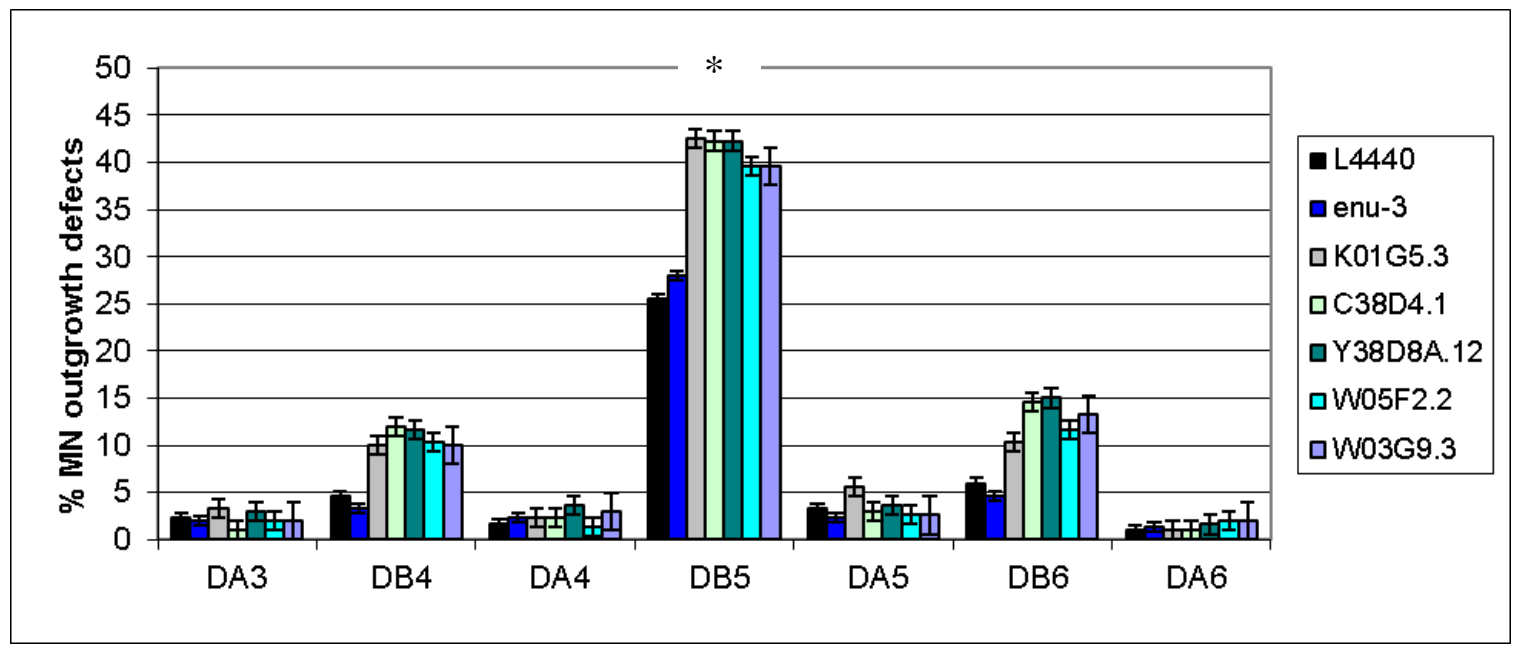

Figure 3.5 RNAi knockdown of the ENU-3 protein family enhances motor neuron outgrowth defects of enu-3(tm4519);unc-6(ev400) mutants.

enu-3(tm4519);unc-6(ev400) mutants were treated with the empty RNAi vector L4440 or the vector containing the RNAi clone specified, and F2 progeny were scored for outgrowth defects. The average score from the three trials performed $(n=100 /$ trial) and standard errors were plotted. $* p$ value less than $0.05, * * p$ value less than $0.01, * * * p$ value less than 0.001 on a chi squared test. 


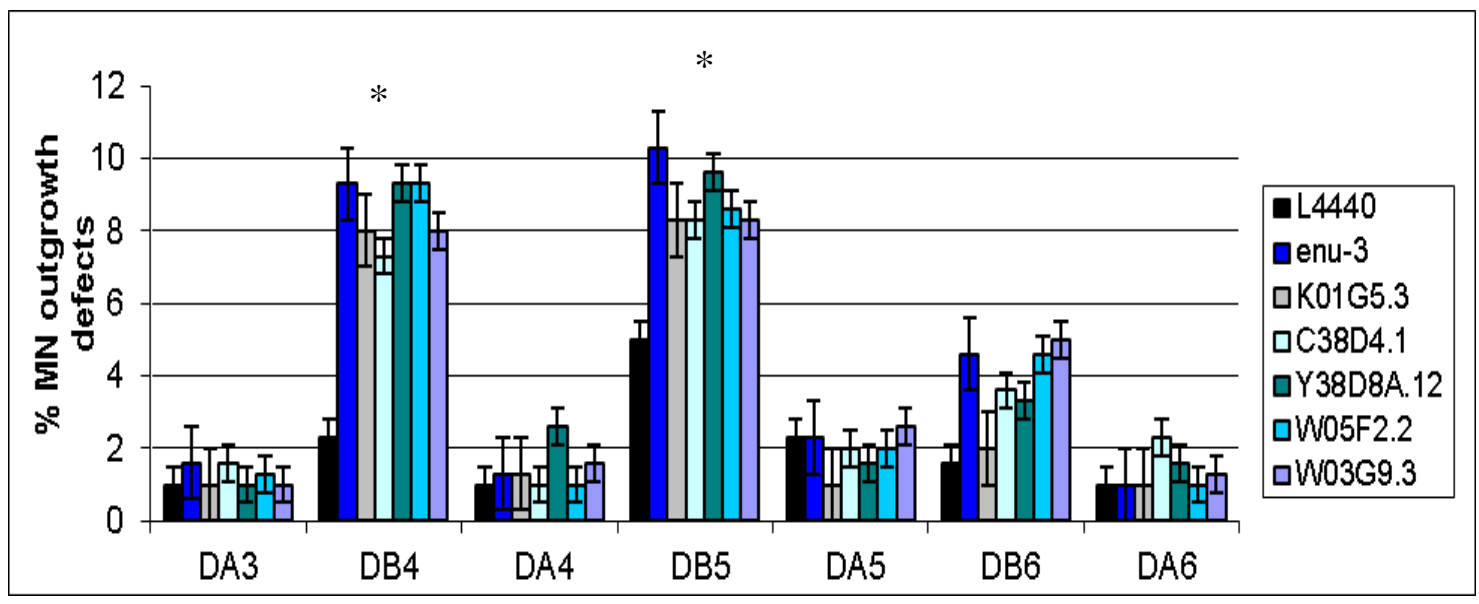

Figure 3.6: RNAi knockdown of the ENU-3 protein family enhances motor neuron outgrowth defects in unc-40(e1430);unc-5(e53) mutants.

unc-40(e1430);unc-5(e53) mutants were treated with the empty RNAi vector L4440 or the vector containing the RNAi clone specified, and F2 progeny were scored for outgrowth defects. The average score from the three trials performed $(n=100 /$ trial) and standard errors were plotted. $* p$ value less than $0.05, * * p$ value less than $0.01, * * * p$ value less than 0.001 on a chi squared test. 


\subsection{ENU-3 expresses on the nuclear membrane/ER of HeLa cells}

A closer look at the amino acid sequence of ENU-3 and four of its paralogs revealed a classic bipartite nuclear localization signal, often found in proteins destined for transport into the nucleus or resident proteins of the nuclear membrane (Cooper, 2000). Two basic residues, KK (amino acids 137-138) were followed by 12 other residues, after which three basic residues, RKR (amino acids 151-153) were found. Since the predicted protein structures of the ENU-3 family members depicted above additionally indicate the presence of a predicted trans-membrane domain at amino acids 108 to 128 , the expression pattern of ENU-3 was first studied in mammalian HeLa cells. This was performed in order to gain further insight into its possible functions by observing the subcellular compartments to which the protein localizes. Unlike expression in C.elegans cells, which are relatively small (2-3 microns) (Hope, 1999), and thus would pose greater difficulties in identifying subcellular localization, expression of proteins from other organisms in HeLa cells (15-20 microns) (Cooper, 2000) or other cell types from higher eukaryotes is common among current research due to the feasibility of visualizing specific cell compartments (Adung'a, Gadelha, \& Field, 2013). Expression of proteins

from distantly related organisms in a mammalian cell system is consistent with current research practices, where proteins of far more distantly related organisms have been investigated using model cell systems of higher eukaryotes (Adung'a, Gadelha, \& Field, 2013). Thus, ENU-3 cDNA and the mammalian expression vector pcDNA3 were amplified and ligated through homologous recombination in competent DH5- $\alpha$ bacterial cells. An additional seamless cloning step through homologous recombination was 
performed as described in Materials and methods in order to add a FLAG tag at the Nterminus of ENU-3 immediately after the predicted signal peptide at amino acid 23. This plasmid was transfected into wild-type HeLa cells and immunofluorescence analysis revealed that FLAG::ENU-3 localized to the nuclear membrane, continuous with the ER of HeLa cells as shown in Figure 3.7. This result was surprising as it was hypothesized that ENU-3 is a plasma membrane protein, possibly a co-receptor of UNC-40, as suggested by the mechanosensory neuron migration defects data as well as the predicted structure of ENU-3 indicative of an extra-cellular region. Nonetheless, multiple transfections of FLAG::ENU-3 showed consistent expression on the nuclear membrane and the ER of HeLa cells. 


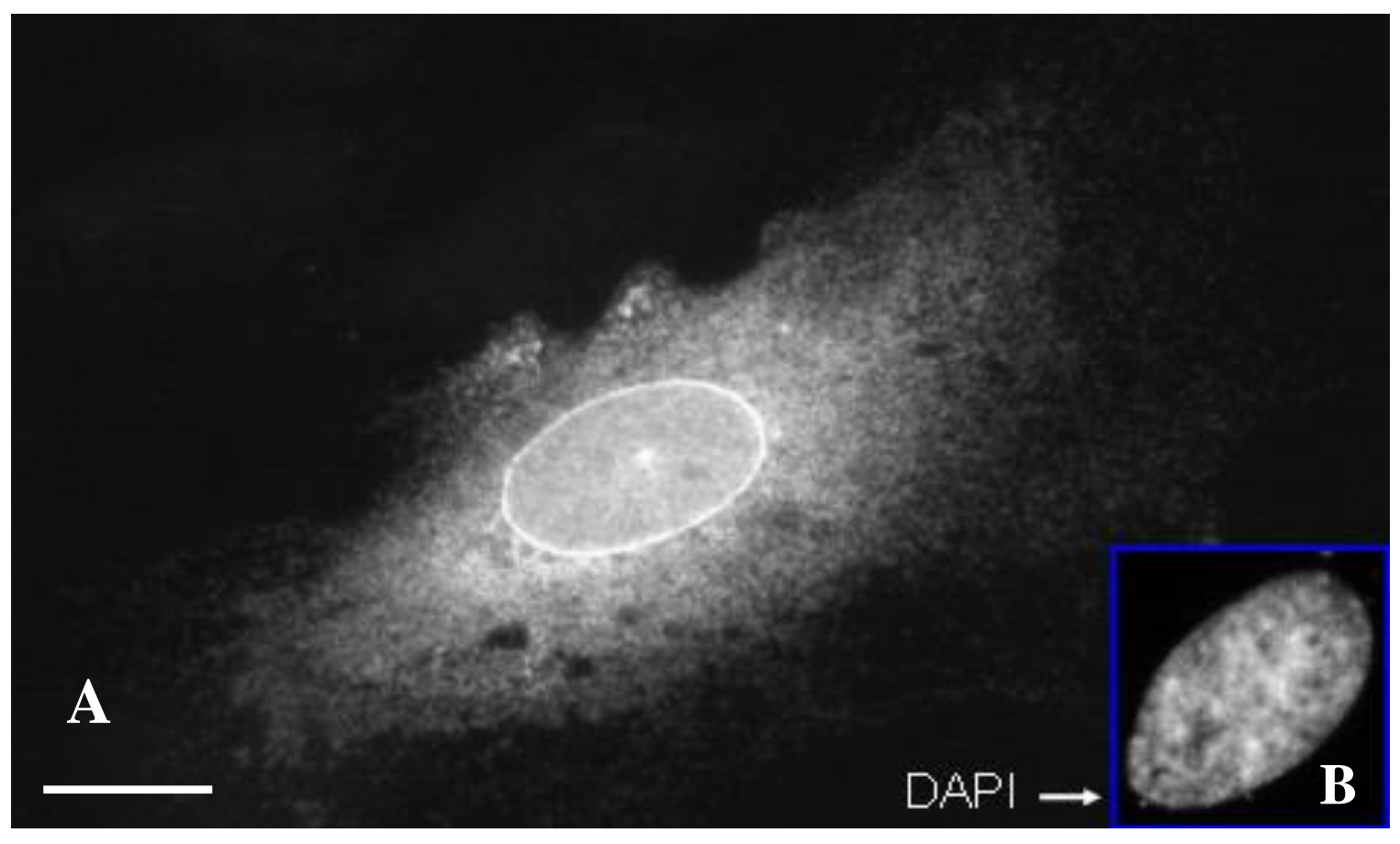

Figure 3.7: FLAG::ENU-3 is expressed in the ER and nuclear membrane in HeLa cells

HeLa cells were transfected with FLAG::ENU-3 and the protein was expressed primarily on the nuclear membrane and in a network pattern typical of the ER (A). Cells were stained with anti-FLAG antibody and Cy3 secondary antibody. DAPI staining the nucleus is shown in panel B. Scale bar, $10 \mathrm{um}$. 
Since the expression of FLAG::ENU-3 in HeLa cells was unexpected due to the predicted extra-cellular region, this led to the question of whether the FLAG tag was causing the retention of ENU-3 in the ER. Thus, two new plasmids were constructed by a similar procedure using seamless cloning through homologous recombination where GFP was inserted at the C-terminus of the new constructs, FLAG::ENU-3::GFP and ENU$3::$ GFP. If the FLAG tag interfered with the true localization of ENU-3, then these two constructs should have been detected in a different location compared to that of FLAG::ENU-3. Multiple transfections with the new plasmids revealed identical expression patterns between FLAG::ENU-3 and the ENU-3 constructs with the additional GFP tag, FLAG::ENU-3::GFP (Figure 3.8) and ENU-3::GFP (Figure 3.9). More importantly, FLAG::ENU-3 and ENU-3::GFP generated similar expression patterns indicating that the expression pattern of ENU-3 was likely to be authentic. The reticular expression was clearly identified, indicative of the ER, and the nuclear membrane outline was noticeably seen in all three plasmids. Thus, the FLAG tag did not interfere with the true localization of ENU-3. Similar expression was observed when transfection was performed in Retinal Pigment Epithelial cells (RPE cells) (data not shown). 


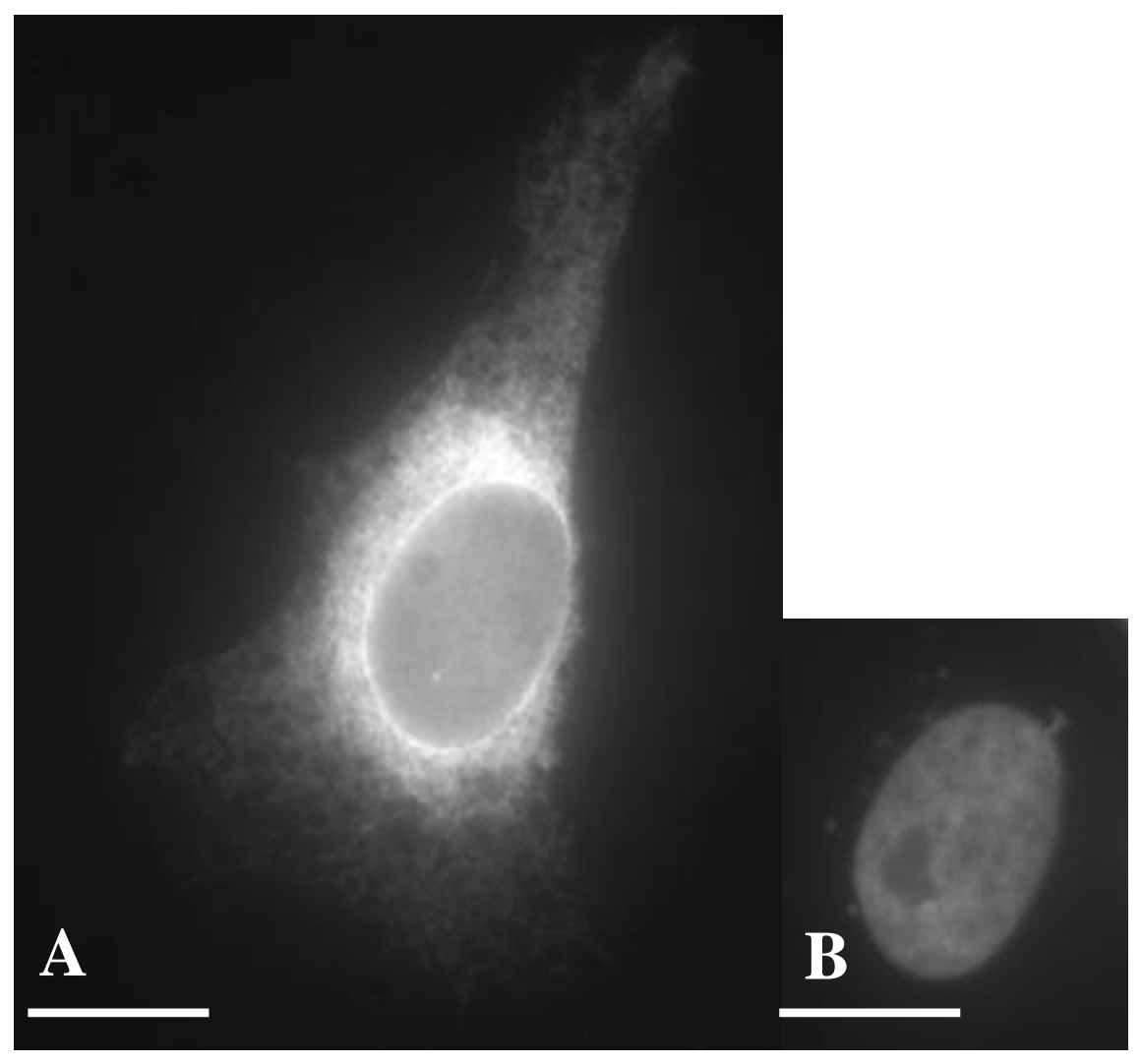

Figure 3.8: FLAG::ENU-3::GFP is expressed in the ER and nuclear membrane in HeLa cells

HeLa cells were transfected with FLAG::ENU-3::GFP and the protein was expressed primarily on the nuclear membrane with a high intensity in the perinuclear region and a network pattern typical of the ER (A), similar to FLAG::ENU-3. Cells were visualized through the GFP channel. DAPI staining the nucleus is shown in panel B. Scale bar, 10um. 


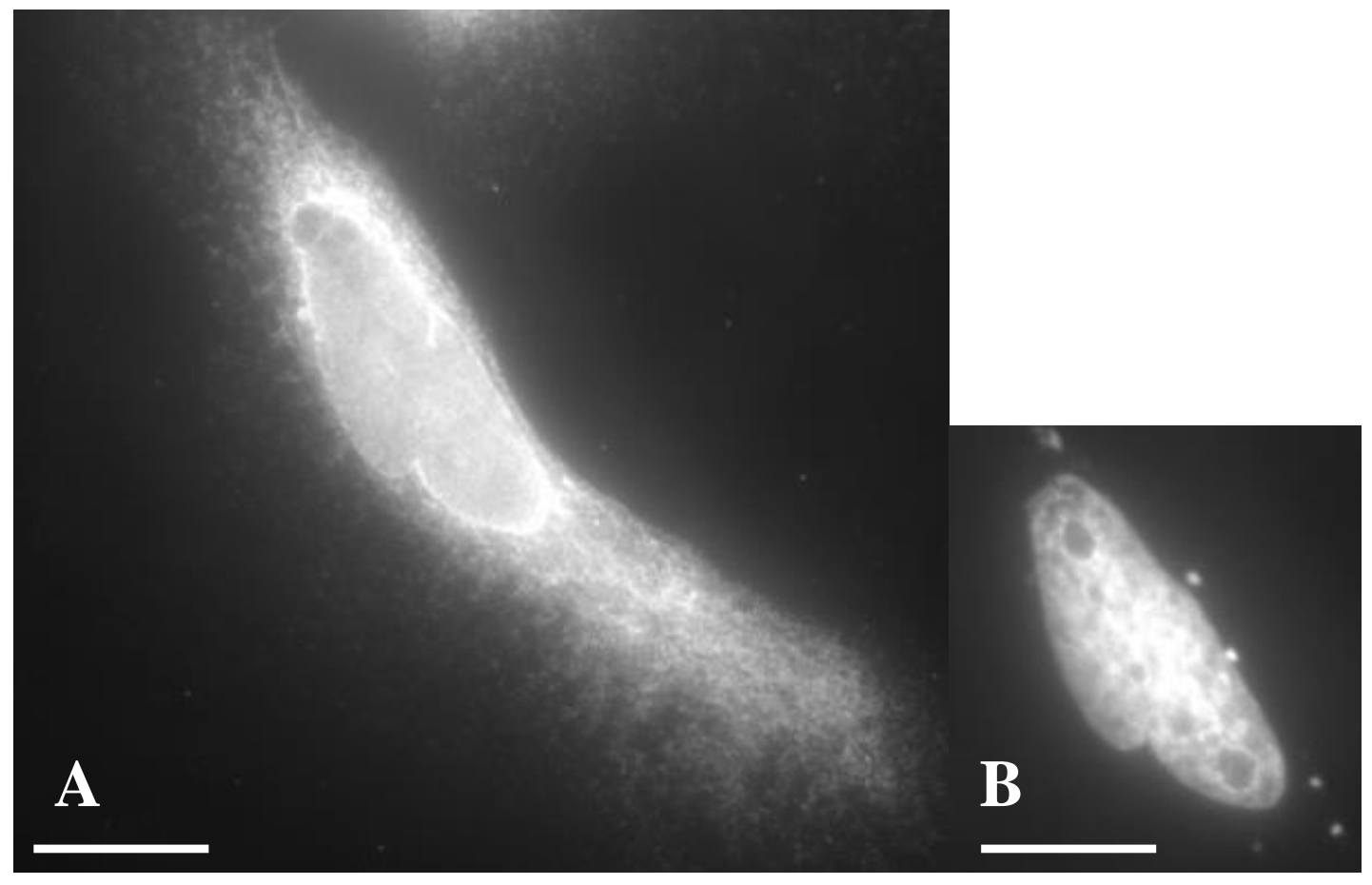

Figure 3.9: ENU-3::GFP is expressed in the ER and nuclear membrane in HeLa cells

HeLa cells were transfected with ENU-3::GFP and the protein was expressed primarily on the nuclear membrane, with a high intensity in the perinuclear region and a network pattern typical of the ER (A), similar to FLAG::ENU-3. Cells were visualized through the GFP channel. DAPI staining the nucleus is shown in panel B. Scale bar, 10um. 
An additional experiment was performed to verify the presence of an extracellular region of ENU-3, as predicted by the SMART database. After transfection with FLAG::ENU-3::GFP, the cells were immunostained with anti-FLAG or anti-GFP antibodies before permeabilization in order to detect any fluorescence outside the cell accountable to ENU-3. Unlike the pearmeabilized control cells which maintained the nuclear membrane/ER expression pattern, fluorescence was not observed with the cells that were non-permeabilized before the primary antibody was added. Thus, it can be concluded that ENU-3 is not largely localized to the plasma membrane given that any possible localization of ENU-3 to the plasma membrane was below detection.

\subsection{F::ENU-3::GFP and ENU-3::GFP have identical molecular weights}

In order to further confirm that the expression observed on the nuclear membrane and ER was not that of only the FLAG tag cleaved with the signal peptide, as opposed to the FLAG tag fused to the ENU-3 protein, the molecular weight of FLAG::ENU-3::GFP and ENU-3::GFP were verified. A 10\% SDS-PAGE gel was run as previously described and a Western Blot with GFP antibody showed that both constructs had an expected molecular weight of approximately $45 \mathrm{kDa}$ (Figure 3.10). ENU-3 is a $19.9 \mathrm{kDa}$ protein excluding its signal peptide which is cleaved off, while the GFP and FLAG tags weigh 26 $\mathrm{kDa}$ and $0.88 \mathrm{kDa}$, respectively. Since the FLAG tag is relatively small, the $0.8 \mathrm{kDa}$ difference in weight between FLAG::ENU-3::GFP and ENU-3::GFP was not detectable. Nonetheless, Figure 3.12 showed that the two proteins are identical in the expected molecular weight, and that the FLAG tag did not interfere with the true localization of ENU-3 in mammalian HeLa cells. 


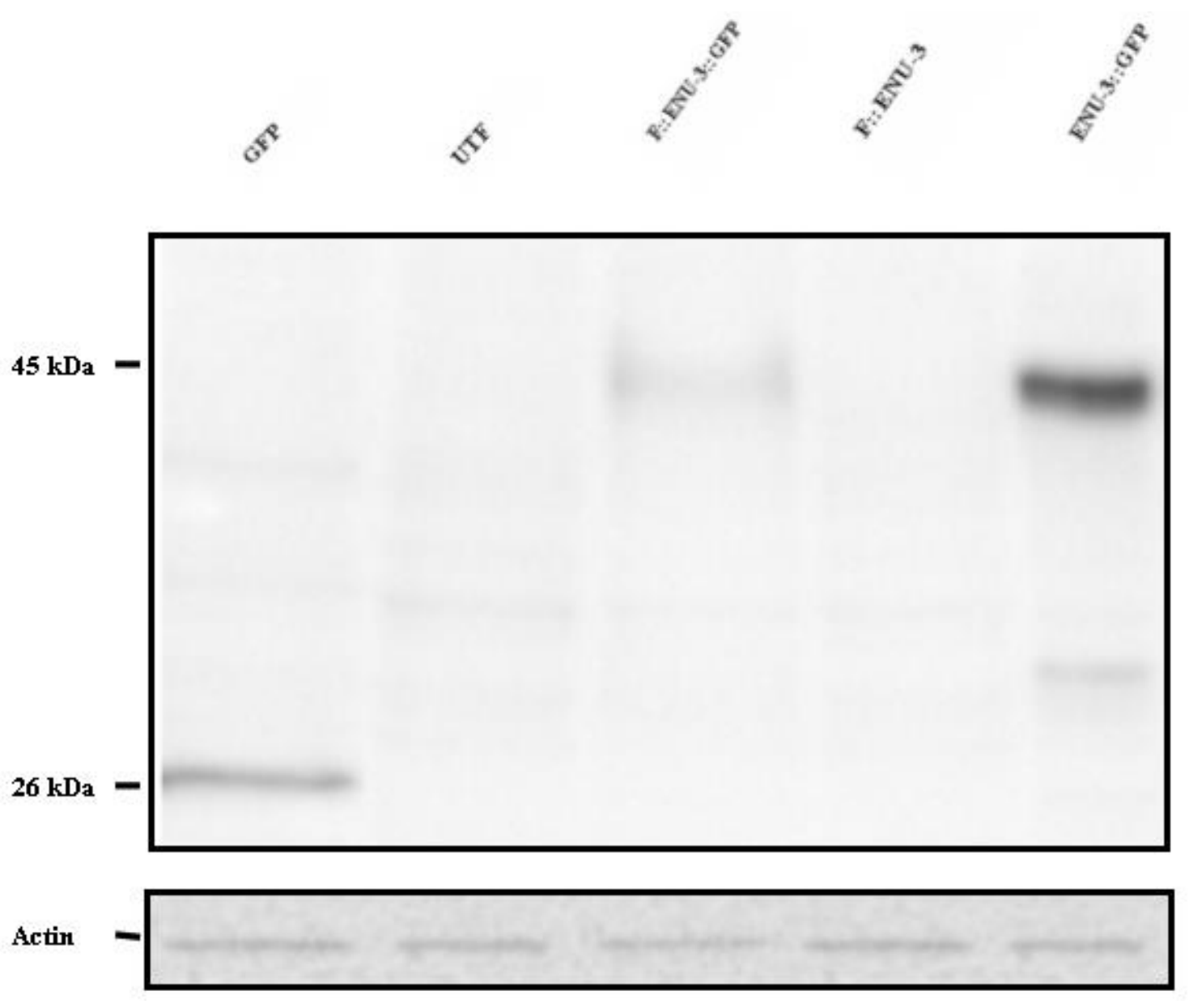

Figure 3.10: Western Blot anti-GFP confirms the molecular weight of ENU-3

Western Blot with GFP antibody detection of F::ENU-3::GFP and ENU-3::GFP in total lysates of transfected HeLa cells. The similarity in molecular weight, as expected at approximately $45 \mathrm{kDa}$, is shown. Lysate from the cells expressing the empty cGFP vector was used as a positive control for detection of GFP, while lysates from the untransfected cells and the cells transfected with FLAG::ENU-3 were used as negative controls. The membrane was additionally immunoblotted for actin to assess loading uniformity. It is possible that ENU-3 tagged with both FLAG and GFP led to an interference of FLAG with the interaction between the primary antibody and GFP. Thus, a lower amount of FLAG::ENU-3::GFP is detected as compared to the single tagged version, ENU-3::GFP. 


\subsection{F::ENU-3 partially co-localizes with ER marker KDEL::GFP}

In order to confirm that ENU-3 localized in the ER, co-transfection of F::ENU-3 and an ER marker, the amino acid sequence, KDEL, fused to GFP (KDEL::GFP) was performed. KDEL is the four amino acid sequence comprised of lysine $(\mathrm{K})$, aspartic acid (D), glutamic acid (E) and leucine (L) that signals a protein to remain trapped in the ER. Proteins containing this sequence will bind to KDEL receptors on the surface of the ER and become internalized into the ER where it will reside (Cooper, 2000) For this reason, despite the fact that the ER is continuous with the nuclear envelope, proteins containing the KDEL sequence will not be detected on the nuclear envelope as they are internalized by KDEL receptors into the ER lumen (Cooper, 2000). After co-transfection, the cells were stained with anti-FLAG antibody, and Cy3 secondary antibody for visualization. The same cells were additionally imaged under the GFP channel in order to confirm colocalization of F::ENU-3 and KDEL::GFP. The perinuclear concentrations of F::ENU-3 and KDEL::GFP largely overlapped and partial co-localization in the ER was observed (Figure 3.11 A-F). However, there were some vesicular structures observed with the KDEL::GFP expression that were not seen with the FLAG::ENU-3 expression. Moreover, it was evident that FLAG::ENU-3 was also expressed on the nuclear membrane, while KDEL::GFP was not. This indicated that FLAG:ENU-3 was not only consistent with the ER expression of ER marker KDEL::GFP as shown by the color merge in Figure 3.11 , but that it also primarily expressed on the nuclear membrane of HeLa cells. This reinforced the idea that the FLAG tag was not responsible for retaining ENU-3 in the ER but that the localization of each of the tagged ENU-3 constructs was indeed due to intrinsic properties of ENU-3 itself. Moreover, it supported the fact that the 
nuclear membrane expression was not an artifact seen in previous expressions of ENU-3 but rather proved that ENU-3 is in fact a nuclear membrane protein which was additionally expressed in the ER as it partially co-localized with the ER marker KDEL::GFP. 

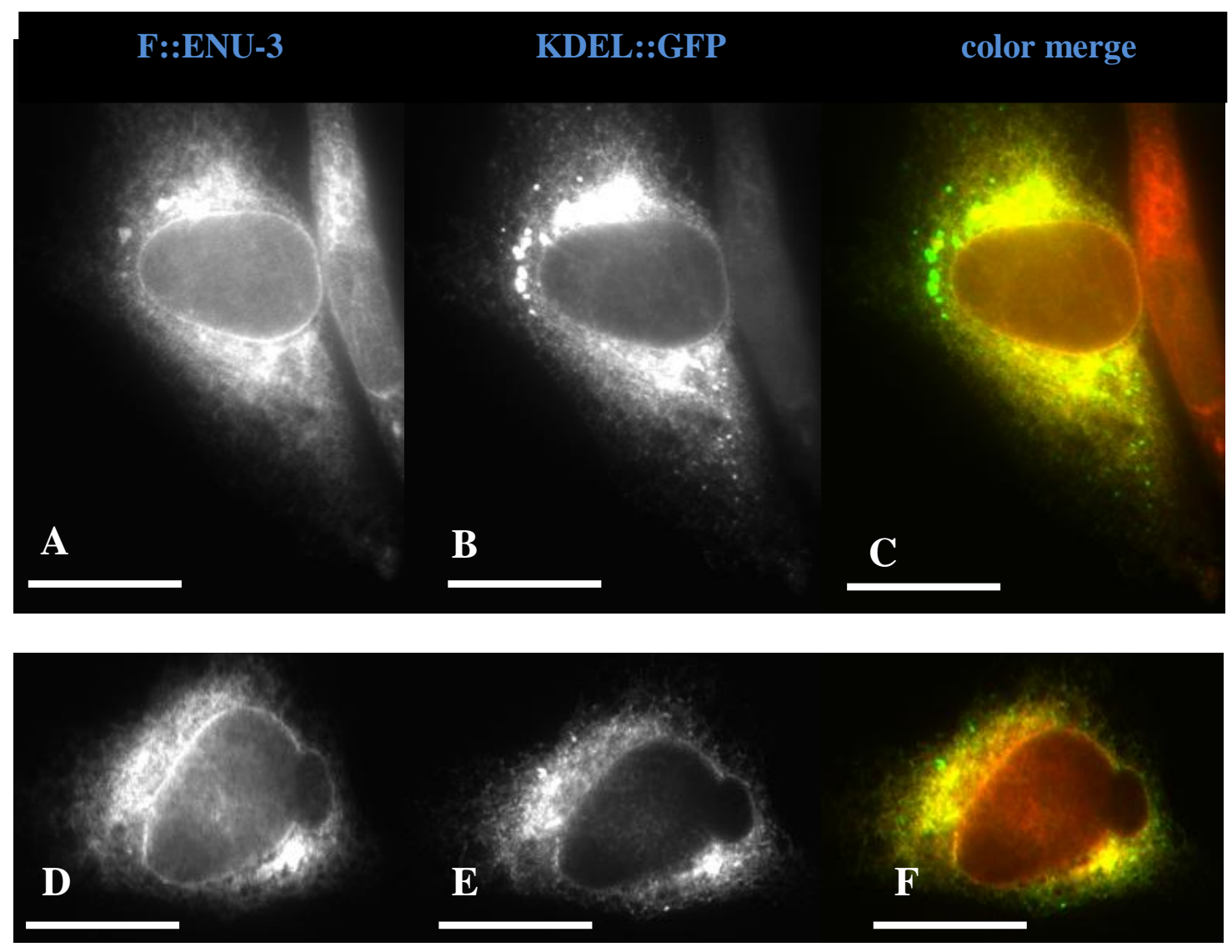

Figure 3.11: F::ENU-3 co-localizes with KDEL::GFP on the ER of HeLa cells

F::ENU-3 was co-transfected with KDEL::GFP in HeLa cells, and stained with anti-FLAG antibody and Cy3 secondary antibody. FLAG::ENU-3 was expressed on the nuclear membrane and the ER (A and D), while KDEL::GFP was expressed on the ER of HeLa cells ( $B$ and E). The color merge between FLAG::ENU-3 (red) and KDEL::GFP (green) in the same cells was evident of partial co-localization in the ER, but not on the nuclear membrane ( $\mathrm{C}$ and F).. Scale bar, 10 um. 


\subsection{Removal of the trans-membrane domain of ENU-3 results in secretion of the protein}

Since the nuclear membrane localization was observed consistently when ENU-3 was expressed in HeLa cells, the importance of the predicted trans-membrane domain was examined. If ENU-3 is in fact inserted into the nuclear membrane necessitating the predicted trans-membrane domain, as expected based on expression analysis thus far, then, the removal of the trans-membrane domain should trigger secretion of the protein from the cell. In order to engineer a $\Delta \mathrm{TM}$ mutant of ENU-3, seamless cloning was utilized as previously when constructing the epitope tagged versions of ENU-3. HeLa cells were transfected with FLAG::ENU-3::GFP and $\triangle \mathrm{TM} F::$ ENU-3::GFP (the predicted trans-membrane domain, from residue 108 to 128 was removed) in order to determine if the predicted trans-membrane domain is essential for localization on the nuclear membrane. Figure 3.12 B,E shows that $\triangle \mathrm{TM}$ F::ENU-3::GFP exhibits localization consistent with being a secreted protein due to a low GFP signal detected in vesicular structures in the ER. This is attributable to the remaining amount of ENU-3 protein in vesicular structures, which was not already secreted from the cell at the time of cell fixation. Consequently, in order to determine if the deletion of the TM domain caused ENU-3 to become a secreted protein as opposed to a TM protein, treatment with Brefeldin A (BFA) was administered at $100 \mathrm{ng} / \mathrm{ml}$ for 12 hours. BFA inhibited transport of secreted proteins from the ER to the Golgi, thus leading to an accumulation of proteins in vesicles within the ER and possible collapse of the Golgi stacks (Misumi et al., 1986). Full length, wild-type FLAG::ENU-3::GFP was unaffected by BFA treatment and maintained its nuclear membrane and reticular ER expression (Figure 3.12 A,D). 
Conversely, in the absence of BFA, there was a low GFP signal detected for $\triangle \mathrm{TM}$ F::ENU-3::GFP in vesicular structures, indicative of the fact that ENU-3 without its trans-membrane domain naturally followed in the secretory pathway of the cell, with a small amount still observed in vesicles (Figure $3.12 \mathrm{~B}, \mathrm{E}$ ). After BFA treatment, there was a dramatic increase in the GFP signal detected for $\triangle \mathrm{TM} F:: \mathrm{ENU}-3:$ GFP, as BFA hindered the secretion of ENU-3, thus leading to an accumulation of the protein in the ER and vesicular structures (Figure $3.12 \mathrm{C}$,F). In addition, $\Delta \mathrm{TM}$ F::ENU-3::GFP did not express on the nuclear membrane and instead demonstrated increased vesicular formation and accumulation of the secreted protein in the ER. These findings suggest that the transmembrane domain of ENU-3 was necessary for insertion into the nuclear membrane of HeLa cells, avoiding the secretory pathway. 


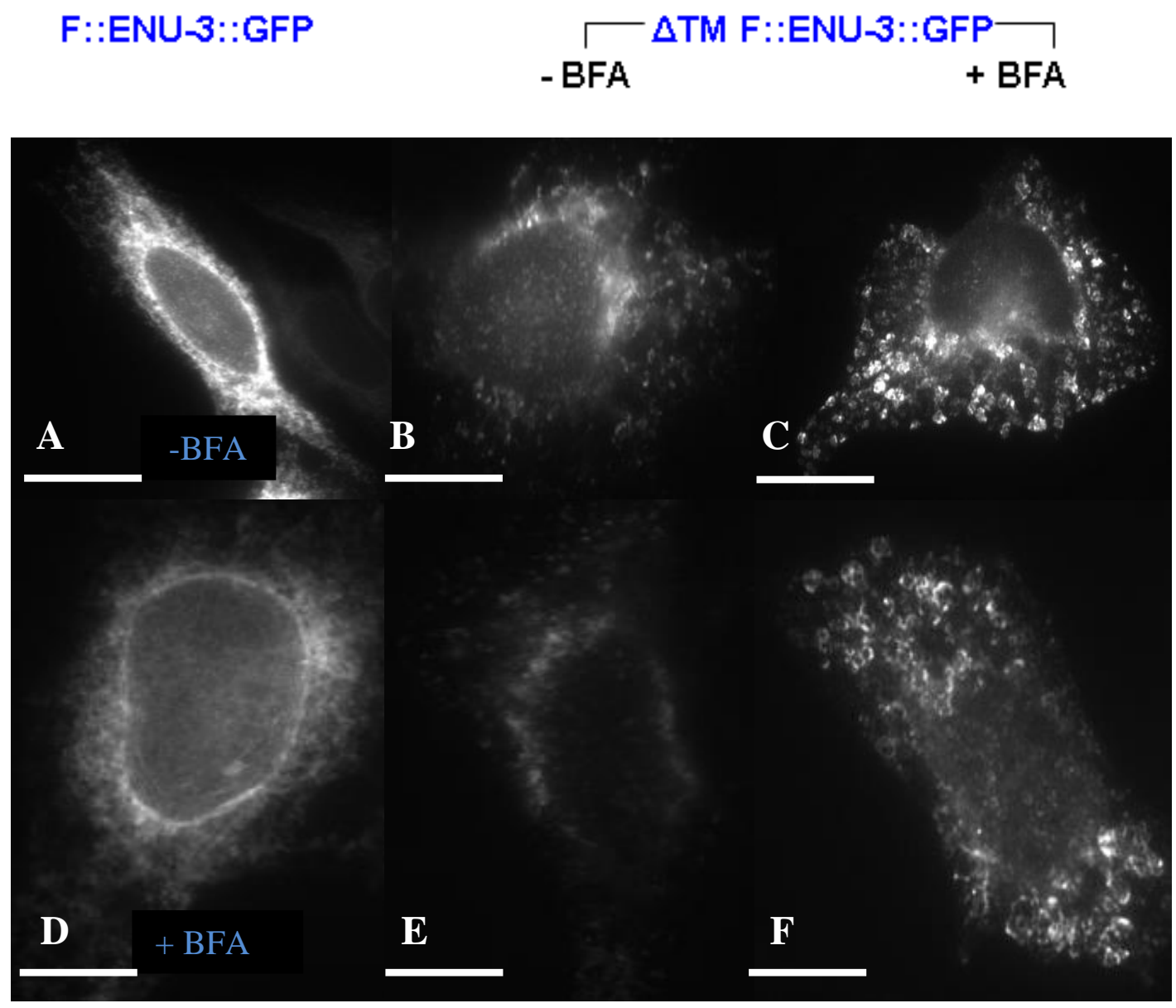

Figure 3.12: Brefeldin A inhibits secretion of $\triangle \mathrm{TM} F:: \mathrm{ENU}-3:: \mathrm{GFP}$ from the ER

HeLa cells were transfected with F::ENU-3::GFP and $\Delta$ TM F::ENU-3::GFP which did not contain the trans-membrane domain, followed by treatment with BFA at $100 \mathrm{ng} / \mathrm{ml}$ for 12 hours. F::ENU-3::GFP maintained the nuclear membrane/ER expression without BFA treatment (A) and this was not affected bv treatment with BFA (D). $\triangle \mathrm{TM} F:: \mathrm{ENU}-$ 3::GFP without BFA treatment $(\mathrm{B}, \mathrm{E})$ was naturally following the secretory pathway indicative of a low GFP signal attributable to ENU-3 and vesicular formation. Addition of BFA inhibited ENU-3 secretion, increased the GFP signal and enhanced accumulation of ENU-3 indicative of greater vesicular formation in the ER (C, F). Scale bar, 10 um. 


\subsection{C38D4.1 paralog expresses on the nuclear membrane/ER of HeLa cells}

Based on the expression analysis of ENU-3 in mammalian cells, it was expected that the four paralogs with a high similarity in protein structure, which were also predicted to contain a trans-membrane domain similar to ENU-3, would also express on the nuclear membrane in HeLa cells. In order to test this, one representative paralog was chosen, C38D4.1 to be expressed in HeLa cells. The cDNA open reading frame of this paralog and the pcDNA3 mammalian expression vector were amplified using an identical gradient PCR setting as previously mentioned. After digestion of the DNA with DpnI for removal of methylated cDNA indicative of the original PCR template, the insert and the vector were transformed into competent DH5- $\alpha$ bacterial cells, in which homologous recombination generated the plasmid of interest. A second step of homologous recombination was performed in order to add the FLAG tag at the N-terminus of C38D4.1 immediately after the signal peptide at amino acid 23. The F::C38D4.1 plasmid was then transfected into wild-type HeLa cells as formerly described and the expression pattern previously observed, that of an emphasized nuclear membrane localization as well as the ER was generated as shown in Figure 3.13. This consistent observation suggested that the predicted trans-membrane domain of C38D4.1 was essential for anchoring the protein into the nuclear membrane, thus C38D4.1 was also a nuclear membrane/ER localized protein, identical to its structurally similar paralog, ENU-3. Furthermore, it was concluded that the expression patterns of the remaining paralogs which contained the predicted trans-membrane domain would be similar to the ones observed for ENU-3 and C38D4.1, however future expression experiments will confirm this prediction. 


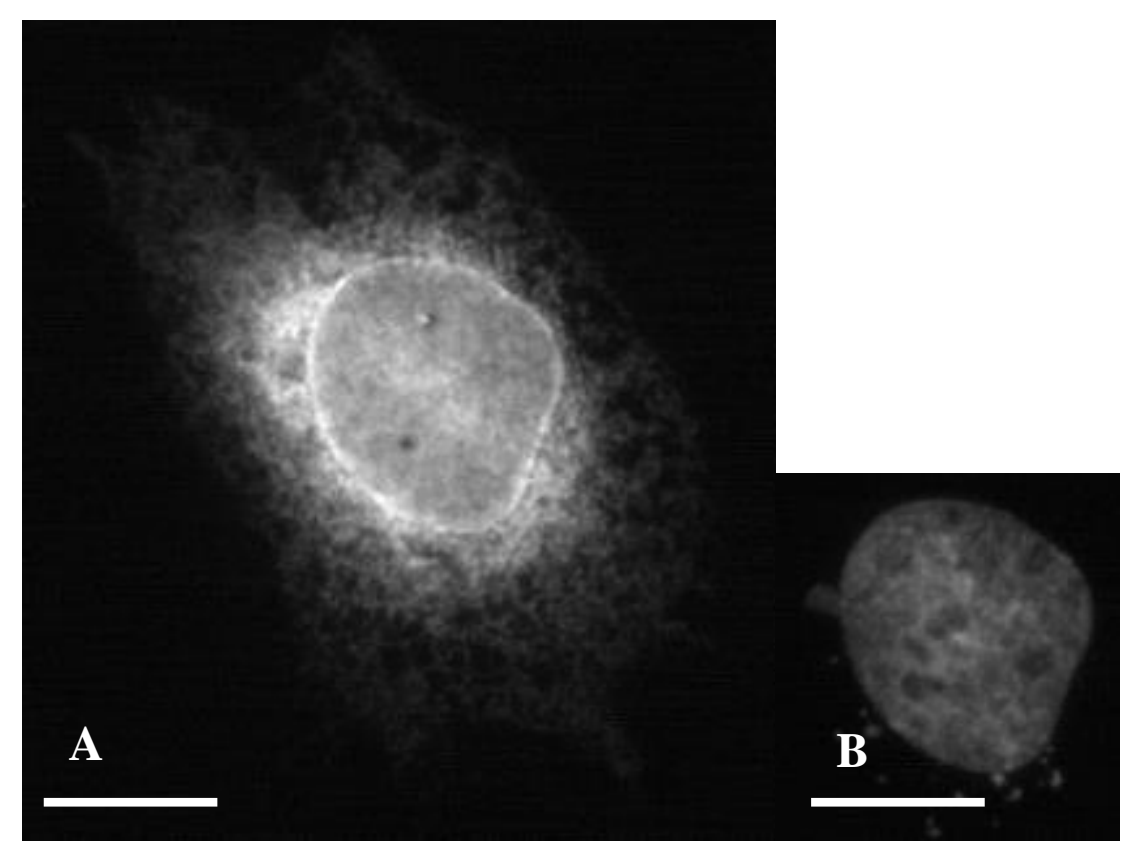

Figure 3.13: F::C38D4.1 is expressed in the ER and nuclear membrane in HeLa cells

HeLa cells were transfected with FLAG::C38D4.1 and the protein was expressed primarily on the nuclear membrane and ER (A). Cells were stained with anti-FLAG antibody and Cy3 secondary antibody. DAPI staining the nucleus is shown in panel B. Scale bar, 10 um. 


\subsection{K01G5.3 paralog is a plasma membrane associated protein}

It was important to additionally consider the expression pattern of K01G5.3 in mammalian cells as this paralog does not contain the predicted trans-membrane domain present in the rest of the ENU-3 protein family members. Thus, a similar procedure of seamless cloning through homologous recombination was followed to generate the plasmid which contained the amplified K01G5.3 cDNA insert and the amplified mammalian expression vector, after transformation into competent DH5-alpha bacterial cells. An additional homologous recombination step was performed in order to add the FLAG tag at the N-terminus immediately after the first predicted coiled-coil region at amino acid 10. The F::K01G5.3 plasmid was transfected into wild-type HeLa cells as previously described. As expected, this paralog did not localize on the nuclear membrane and ER, as observed with ENU-3 and C38D4.1 since it was not predicted to contain a trans-membrane domain. Figure 3.14 shows that F::K01G5.3 is likely to be a plasma membrane associated protein with noticeable expression in microspikes structures in HeLa cells. It was evident that F::K01G5.3 was expressed intracellularly throughout the cytoplasm, as expected given its predicted structure that lacked a transmembrane domain. It was concluded that K01G5.3 may be an intracellular plasma membrane associated protein and that K01G5.3 may modify actin dynamics, as the microspikes structures around the inside of cell surface, indicative of lamellipodium and filopodia, are noticeably labelled. Future experiments including staining of actin in untransfected cells and cells transfected with F::K01G5.3, will test whether K01G5.3 modifies actin assembly. 

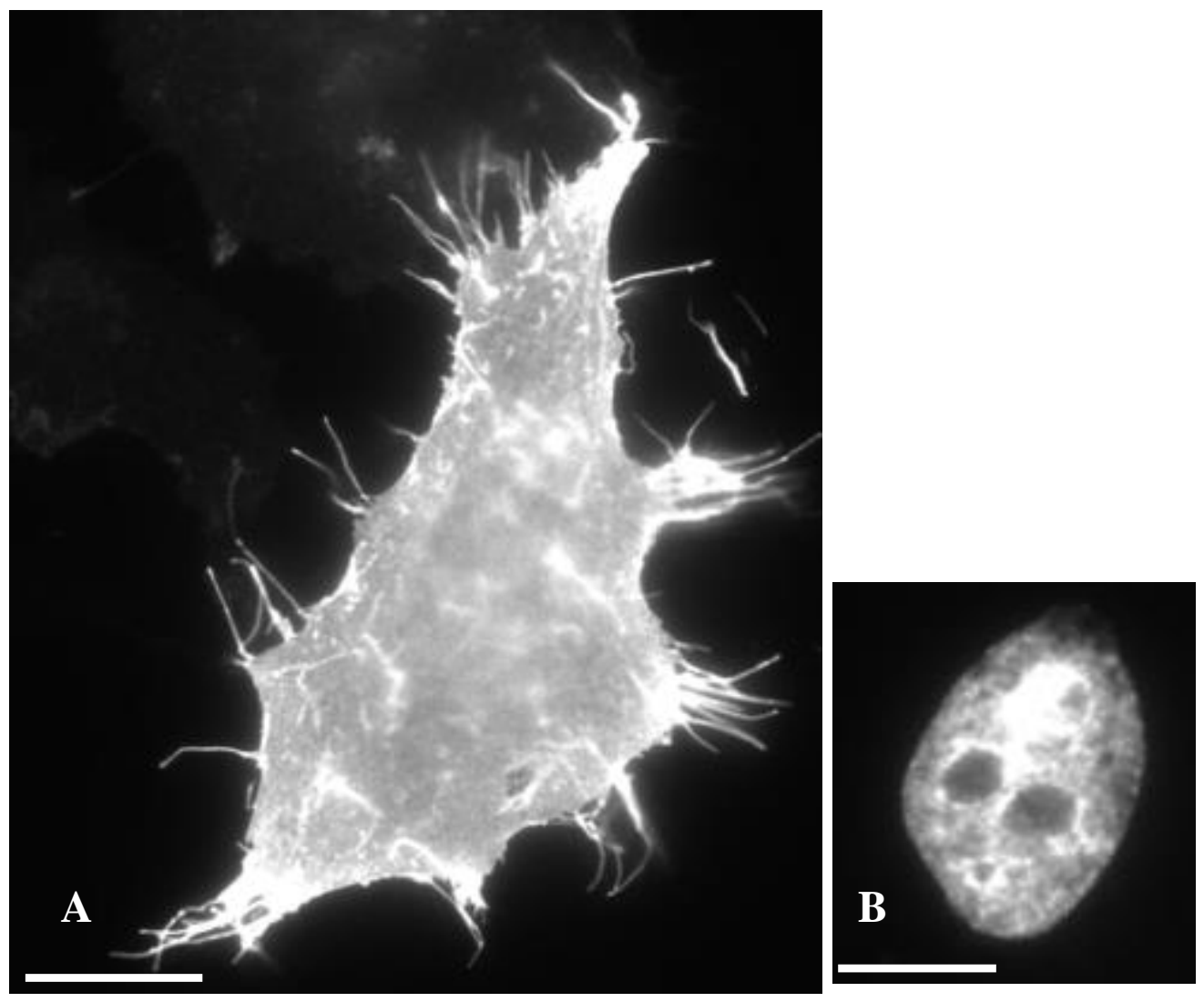

Figure 3.14: Expression pattern of F::K01G5.3 in HeLa cells

HeLa cells were transfected with FLAG::K01G5.3 and the protein was expressed intracellularly throughout the cytoplasm and appears to be a plasma membrane-associated protein (A). Cells were stained with anti-FLAG antibody and Cy3 secondary antibody. DAPI staining the nucleus is shown in panel B. Scale bar, $10 \mathrm{um}$. 


\section{Chapter 4. Discussion}

The main objective of this thesis was to further investigate possible pathways in which a novel protein, ENU-3 may function to affect motor neuron outgrowth and guidance of the mechanosensory neurons in C. elegans. Moreover, ENU-3 is a member of the ENU-3 protein family which includes 5 additional paralogs highly similar in amino acid structure, of unknown function. Thus, their roles in directing axon outgrowth and guidance of neurons in C. elegans were examined. It is important to note that guidance of AVM and PVM neurons in the ventral direction was previously only known to be affected by the UNC-6/Netrin and SLT-1/Slit pathways, thus whether ENU-3 functions in those pathways or parallel to them was of interest. Similarly, regulation of the outgrowth of the axons of the dorsal migration of the DA and DB subset of motor neuron axons is only known to be affected by the UNC-6/Netrin pathway. However, mutants with an absence of either the UNC-6 ligand or the UNC-5 receptor do not exhibit complete penetrance of defects suggesting that other pathways must be controlling dorsal guidance of axons. Due to the presence of a predicted nuclear localization signal and a transmembrane domain for ENU-3 and four of its paralogs, this thesis also focused on expression analyses in mammalian HeLa cells to further characterize the sub-cellular localization of ENU-3 and its paralogs. 


\subsection{ENU-3 functions in a pathway dependent on UNC-6/Netrin and parallel to UNC-}

\section{0 to enhance guidance defects of touch receptor neurons}

Construction of genetic knockouts of ENU-3 in unc-40(e1430) null mutants revealed a significant enhancement of AVM and PVM guidance defects. RNA interference knockdown data additionally supported this finding as a reduction of enu-3 mRNA in the two unc-40 mutants and a reduction of unc-40 mRNA in enu-3 mutants enhanced the guidance defects of AVM and PVM touch receptor neurons (Yee et al., 2014). Together, these results suggest that ENU-3 works independently and parallel to UNC-40 to control guidance of the mechanosensory neurons in C. elegans. Current understanding of ventral guidance demonstrates that UNC-40 is the only known receptor of the UNC-6 ligand functioning in the ventral ward direction (Chan et al., 1996). However, unc-40 mutants have less guidance defects than unc- 6 null mutants. This indicates that there must be a pathway downstream of UNC-6 working in part redundantly with UNC-40. Since enu-3 did not enhance the guidance defects of unc-6 null mutants, this further supports the idea that ENU-3 functions dependently and downstream of UNC-6. The fact that ENU-3 functions in the UNC-6/Netrin pathway is additionally supported by an enhancement of guidance defects when enu-3 is knocked out in a slt-1 null mutant. Since enu-3 does not enhance unc-6 null mutants but does affect slt-1 mutants, then this implies that ENU-3 does not function in the SLT-1/Slit pathway but rather in the only other known pathway, the UNC-6/Netrin pathway. Furthermore, the double mutants of unc-40(e1430);enu3(tm4519) and enu-3(tm4519);unc-6(ev400) have a similar amount of guidance defects, suggesting that ENU-3 may be working in the redundant, parallel pathway downstream of UNC-6. Redundancy is essential as two or more pathways often compensate for each 
other leading to robustness against failure of a single pathway. A targeted inhibition of a specific pathway will not lead to complete failure of all components involved as additional, redundant pathways, with diverse sensitivities will respond differently and will not all be affected. All in all, the genetic knockout and RNAi knockdowns demonstrated that it is possible that ENU-3 is in the pathway of another receptor parallel to UNC-40 and downstream of UNC-6 that can detect and respond to ventral source of UNC-6 in order to affect guidance of AVM and PVM touch receptor neurons as shown in the ventral guidance signalling model in Figure 4.1. 


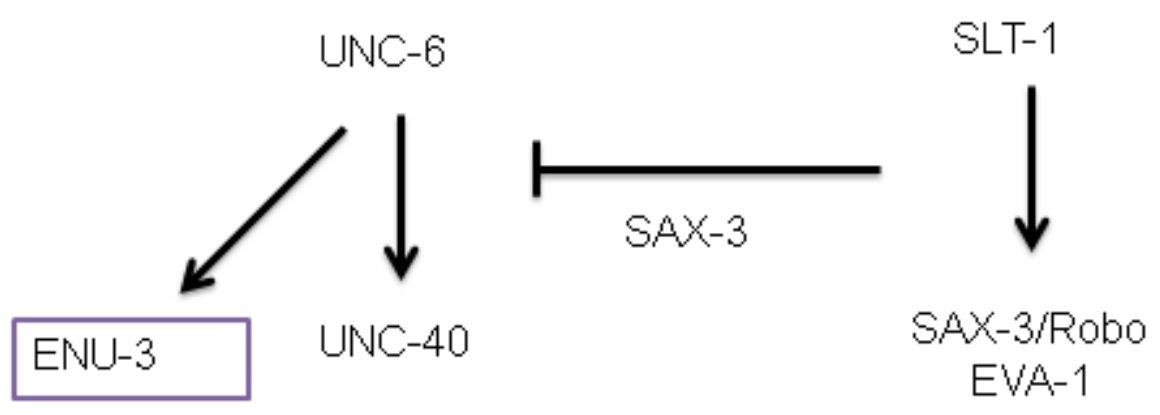

Figure 4.1: Ventral guidance signalling model in C. elegans

ENU-3 appears to work independently of the SLT-1 pathway components, and consequently, in the UNC-6/Netrin pathway, dependent on the presence of UNC-6 and downstream of it. ENU-3 may function in the pathway of another, unidentified receptor, parallel to UNC-40 that can respond to sources of UNC-6 and affect guidance of touch receptor neurons in C. elegans. 
In contrast, knockdown by RNAi of the ENU-3 paralogs did not enhance guidance defects of unc- 40 mutants. This was surprising at first as the protein structure similarity between the ENU-3 protein family members is high suggesting the possibility of similar roles. RNAi reductions of mRNA corresponding to individual paralogs did not appear to enhance the AVM and PVM guidance defects, except for enu-3. Although RNAi does not completely reduce the protein levels produced, it proves to be a rapid and reliable indication of trends observed from genetic knock-outs of genes. Nevertheless, it was concluded that the paralogs, unlike ENU-3 do not function in the UNC-6/Netrin pathway to affect migration of touch receptor neurons in $u n c-40$ mutants. Perhaps the longer predicted C-terminal intracellular region of all paralogs absent in ENU-3 provides the paralogs with different roles. At first glance it would appear that since all the paralogs except ENU-3 possess the long carboxyl terminal tail they are unable to participate for some reason in the guidance of AVM and PVM leaving this role to ENU-3 alone. Databases searching for conserved domains in proteins sometimes indicate that the longer carboxyl terminal tails may contain a protease region towards their C-terminal end that is absent in ENU-3 so perhaps this is significant in their lack of effect on unc-40 null mutants.

Asymmetric localization of receptors on the surface of growth cones contributes to proper guidance of axons mediated by interactions between the receptors and their respective ligands (Tong et al., 2011). For instance, UNC-40 receptors have been shown to be mostly localized along the developing side of HSN (hermaphrodite-specific neuron) (Adler et al., 2006, Xu et al., 2009, reviewed in Killeen and Sybingco, 2008). When UNC-5 binds to UNC-6, tyrosine kinase phosphorylates the UNC-5 receptors (Tong et 
al., 2001; Killeen et al., 2002), however mutations in any of the sites of phosphorylation have been linked to axon guidance defects (Killeen et al., 2002). It has been demonstrated that SRC-1, a receptor tyrosine kinase functions downstream of UNC-40 to regulate UNC-5 signalling by directly interacting with a region of UNC-5 causing its phosphorylation (Li et al., 2004, Lee et al., 2005). The direct interaction between SRC-1 and UNC-40 (Li et al., 2004, Liu et al., 2004) has been shown to be involved in affecting the phosphorylation of the UNC-5 receptor, activating intracellular signalling which results in growth cones expressing UNC-5 receptors to migrate away from sources of UNC-6. In contrast, the same interaction is imperative for asymmetric UNC-40 localization on cells observed in HSN (Adler et al., 2006, Xu et al., 2009), and in AVM and PVM neurons (Xu et al., 2009), leading to the attraction between growth cones expressing UNC-40 receptors and sources of UNC-6. It appears that UNC-40 is able to promote attraction and repulsion responses depending on the way it interacts with downstream receptor tyrosine kinases such as SRC-1 (Chang et al., 2004, Lee et al., 2005).

It is evident that there must be other mechanisms involved in directing the asymmetric expression of UNC-40 receptors and stochastic localization of UNC-5 receptors on the growth cones of developing neurons (Wadsworth, 2002). Enhanced guidance defects observed from genetic knock-out and RNAi knock-down of enu-3 in unc-40 mutants suggest that ENU-3 may function to direct proper organization and positioning of at least the UNC-40 receptors on the surface of the AVM and PVM cells. Animals with mutations in both $u n c-40$ and enu-3 appear to have a significant amount of axons that fail to migrate ventrally and instead they are directly projected towards the 
head. It is plausible that in the absence of ENU-3, the UNC-40 receptors are not localized asymmetrically towards the side of the cell facing the ventral cord which would allow it to migrate ventrally towards the source of UNC-6 along the VC. Instead, without functional ENU-3, UNC-40 receptors are misplaced possibly along the sides of the cell or the side facing the dorsal cord. Without correct localization of UNC-40 receptors relative to its ligand, UNC-6, an attraction response will not be activated, accounting for the misguided axons often seen in unc-40(e1430);enu-3(tm4519) double mutants. Nonetheless, enu-3 single mutants have a low penetrance of AVM and PVM defects, thus its effect on proper localization of UNC-40 receptors may be negligible and surely not as significant as the ability of other proteins like SRC-1 to establish this process. Further experiments will determine the possibility of additional roles ENU-3 may have in guidance of touch receptor neurons.

\subsection{ENU-3 and its paralogs function in a parallel pathway to UNC-6/Netrin to enhance motor neuron outgrowth defects}

Mutations in enu-3 and all its paralogs significantly enhance DA and DB motor neuron outgrowth defects in both $u n c-6$ and $u n c-5$ putative null mutants. Since these proteins are the two members of the only known UNC-6/Netrin signalling pathway to be involved in dorsal guidance of axons, then enu-3 and its paralogs must be working in a parallel pathway to the UNC-6/Netrin signalling pathway to affect migrations of DA and DB developing neurons. Since ENU-3 is predicted to be a trans-membrane protein in addition to genetic knock-out and knock-down data from the touch receptor neuron analysis, then it is possible that ENU-3 is working independently of the UNC-40 and UNC-5 receptors, in a pathway of a another receptor which can respond to sources of UNC-6. 
Hedgecock et al. (1990) showed that both UNC-40 and UNC-5 are necessary for longrange migration of motor neurons towards the DNC. Heterodimer formation of UNC40::UNC-5 is favored on growth cones of developing neurons (Wadsworth, 2002) and the source of UNC-6 along the VNC causes an overall repulsion response and guides these processes dorsally (Wadsworth, 2002). Another gradient molecule localized along the dorsal cord, UNC-129 has been shown to be able to break apart UNC-5 homodimers in favor of UNC-40:UNC-5 heterodimers thus maintaining a balance between the two as UNC-40 is needed for long-range migrations of motor neurons (MacNeil et al., 2009). This mechanism involves UNC-129 binding to UNC-5 which is believed to change the signalling pathway from UNC-5 only signalling to UNC-5 + UNC-40 signaling. The heterodimer formation is more sensitive to low sources of UNC-6 as the growth cone is moving away from the $\mathrm{VNC}$, thus maintaining a repulsive response (MacNeil et al., 2009). This stable and more favorable formation of heterodimers permits processes of motor neurons to achieve correct trajectories and to be able to innervate their respective target muscle along the dorsal nerve cord. It can be postulated that ENU-3 and its paralogs may have a similar function to UNC-129 in the sense that it may also favor formation of UNC-40:UNC-5 heterodimers by breaking up UNC-40 homodimers. If there is a high concentration of UNC-40 homodimers on growth cones, then, they may overcome the repulsion of UNC-5 by UNC-6, and thus will result in a failure of processes to exit the ventral cord as UNC-40 is attracted to UNC-6. Thus there must be proteins involved in preventing the UNC-40 homodimer formation, and based on genetic knockout results, ENU-3 and its paralogs could break up the UNC-40 homodimers and favor formation of UNC-40:UNC-5 heterodimers. Since mutation in enu-3 and its 
paralogs all enhance outgrowth defects, they must be involved in guiding these axons towards the dorsal nerve cord during their development. Although this is a likely role for ENU-3 and its paralogs, breaking up UNC-40 homodimers cannot be the only role they play in affecting axon outgrowth of motor neurons. If that was the case, a triple mutant containing non-functional ENU-3 (or any of its paralogs), UNC-40 and UNC-5, should have no effects on axon outgrowth. However, the genetic knock-out results show that is not true, as there was an enhancement of outgrowth defects especially in DB4, DB5 and DB6 in the triple mutant compared to the unc-40(e1430);unc-5(e53) mutant, but the amount of defects were not as high as in a enu-3(tm4519);unc-5(e53) mutant. These observations indicate that ENU-3 seems to exhibit axon outgrowth activity independently of UNC-40 and UNC-5. Additionally, enu-3 enhanced outgrowth defects of unc-6 mutants suggesting an additional role for ENU-3 and possibly its paralogs independent of all members of the UNC-6/Netrin signalling pathway.

The fact that there is an enhancement of axon outgrowth defects in the unc40(e1430);enu-3(tm4519);unc-5(e53) triple mutants but not to the same degree as observed in the enu-3(tm4519);unc-5(e53) double mutant also suggests that outgrowth defects are dependent on the presence of UNC-40. Furthermore, the outgrowth defects in the unc-6(e78) hypomorph allele mutants were greater than the defects observed in the putative null unc-6(ev400) allele mutant. Lim and Wadsworth (2002) demonstrated that unc-6(e78) mutants have a cysteine to tyrosine substitution mutation in the V-3 domain of UNC-6. It was further postulated that this version of UNC-6 can interact appropriately with UNC-40 but not with UNC-5, accounting for the relatively high percentage of DA and DB motor neuron outgrowth defects in unc-6(e78) mutants. Since this version of 
UNC-6 cannot trigger a repulsion response in growth cones expressing UNC-40::UNC-5 heterodimers, and instead can only interact with UNC-40, causing chemo-attraction towards the ventral cord, then animals with mutations in UNC- 6 encoded by the unc6(e78) version exhibit more outgrowth defects of motor neurons dependent on the presence of UNC-40. Since the double mutant unc-40(e1430);unc-6(e78) appeared to have no outgrowth defects, this supported the idea that the mutation in UNC-6 encoded by unc-6(e78) necessitates the presence of UNC-40 to exhibit a greater effect on outgrowth of motor neurons, especially DB5. Additionally, in order to further test the idea that UNC-40 opposes outgrowth of neurons by binding to UNC-6, and that it can be done without the presence of UNC-5, a double mutant of unc-40(e4130);unc-5(e53) was constructed. As expected, this strain had almost no outgrowth defects further supporting the idea that in the absence of UNC-5, UNC-40 homodimers may be expressed at a higher concentration and are thus attracted towards ventral sources of UNC-6. Indeed, UNC-40 seems to play a significant role in opposing outgrowth defects of motor neurons. This is a promising idea as Lundquist and Norris (2011) also demonstrated that UNC-40 enhanced outgrowth defects of VD and DD motor neurons in the absence of UNC-5.

The results indicating that ENU-3 is working in the UNC-6/Netrin pathway to affect guidance of touch receptor neurons while also working in a parallel pathway to that of UNC-6/Netrin to affect outgrowth of motor neurons make it difficult to assign a concrete role for ENU-3 in the nervous system development of C.elegans. It is plausible to conclude that ENU-3 may be a protein shared by two separate pathways to promote axon outgrowth of motor neurons, one of which is clearly the UNC-6/Netrin pathway and the other a possible novel pathway involving a receptor other than UNC-40. 
Despite the significant effects on outgrowth of motor neurons caused by mutations in all members of the UNC-6/Netrin signalling pathway, UNC-6, UNC-40, UNC-5 and ENU-3 cannot possibly be the only proteins involved in affecting directionality of developing processes. This is supported by both genetic and RNAi knock-outs and knock-downs of combinations of these proteins resulting in outgrowth defects observed in mainly DB4, DB5 and DB6 motor neurons. There are no significant outgrowth defects observed in the remaining DB motor neurons or any of the DA subset of motor neurons indicating the presence of other proteins affecting outgrowth of developing motor neurons. We hypothesized that the five paralogs of ENU-3, highly homologous in protein structure to ENU-3, could be some of the additional proteins involved in axon outgrowth of motor neurons. As expected, RNAi knockdowns of each paralog individually in unc-5, unc-6 and unc-40(e1430);unc-5(e53) mutants resulted in enhancement of defects mainly observed in the DB4 and DB5 motor neurons, similar to the effect of enu-3(tm4519);unc5(e53) mutants. Some of these findings were also confirmed with genetic construction of several double and triple mutants of paralogs in unc-5 mutant backgrounds (personal communication, Hipolito, 2014). Her data support the idea that the ENU-3 proteins function redundantly to each other in supporting motor neuron axon outgrowth in the absence of UNC-5. Furthermore, she found that there were more defects when additional members of the family were depleted either by RNAi or genetically. Together these results suggest that all members of the ENU-3 protein family appear to have a role in affecting axon outgrowth of a subset of motor neurons in $C$. elegans that is independent of UNC-40, UNC-5 or UNC-6, suggesting they have roles in a novel unidentified pathway other than UNC-6/Netrin. 
It is important to note that most of the data involving the paralogs of ENU-3 has been generated through RNAi. Although it has been argued that RNAi is not very efficient in the nervous system of $C$. elegans due to a higher concentration of exoribonuclease I in a subset of neurons which degrades the dsRNA synthesized by T7 polymerase from the RNAi plasmids, sensitivity to RNAi treatment varies among all types of neurons (Timmons, Court, \& Fire, 2001). Specifically, it has been proven that RNAi treatments affect cholinergic, glutamergic and touch neurons to a greater degree than they do GABAergic and dopaminergic neurons (Asikainen et al., 2005). Since DA and DB motor neurons are cholinergic (McIntire et al., 1993), their relatively higher sensitivity to RNAi allowed for a reliable and rapid generation of trends involving axon outgrowth defects. The pattern observed through RNAi multiple knockdowns of ENU-3 protein family members in which more of the DB and DA subset of motor neurons experienced enhanced outgrowth defects as more paralogs were knocked-down is similar to observations made by Heber et al. (2000). As more proteins of the amyloid precursor protein (APP) family were knocked out genetically, they observed an increase in deaths of mutant mice compared to mice that had mutations in a smaller number of the APP proteins (Heber et al., 2000). Nevertheless, the paralogs of ENU-3 undoubtedly play a role in affecting outgrowth of motor neurons, and enhancement of outgrowth defects in the subset of motor neurons, other than in the consistently sensitive DB4, DB5 and DB6, can be achieved by multiple knockdowns of these proteins. Additionally, enhancement of outgrowth defects in unc-5, unc-6 and unc-40(e1430);unc-5(e53) mutants caused by knock-down of all members of the ENU-3 protein family indicated that at least part of their role in axon guidance is not dependent on proteins in the UNC-6/Netrin signalling 
pathway. Consequently, ENU-3 and its paralogs must function in a novel, unidentified pathway to control dorsal guidance of DA and DB motor neurons.

Levy-Strumpf and Culotti (2014) have recently identified a long-sought after additional mechanism involved in regulation of dorsal-ventral guidance of neurons working in parallel to the UNC-6/Netrin signalling pathway. The Wnt pathway essential in anterior-posterior guidance of neurons is not only responsible for regulating axonal development in the anterior-posterior axis of the animal, but through genetic analyses performed by Strumpf and Culotti (2014), it was identified that the Wnt pathway is working in parallel and redundantly to the UNC-6/Netrin pathway to regulate guidance of neurons and the distal tip cells in the dorsal-ventral axis in C.elegans. The distal tip cells use similar guidance molecules as the motor neurons. EGL-20/Wnt ligand which is normally expressed in the posterior side of the animal provides instructive polarity to axons developing in the anterior-posterior axis, such as ALM and PLM which express Frizzled receptors, and chemo-repulse these permitting migrations of growth cones away from the posterior and towards the anterior side of the animal. However, it was shown that simultaneous genetic knock-out of components of both the UNC-6/Netrin and Wnt pathways, led to an enhancement of guidance defects in neurons developing in the dorsalventral axis (Strumpf \& Culotti, 2014). Moreover, the UNC-6/Netrin pathway was also identified to affect guidance of neurons not only in the dorsal-ventral guidance, but also in the anterior-posterior axis (Strumpf \& Culotti, 2014). These findings suggest that the two pathways function in parallel and redundantly to affect migration of neurons in both axes. 
Since the results from the genetic knock-down and knock-outs of ENU-3 and its paralogs in this thesis pointed to the idea that these proteins work parallel to the UNC6/Netrin pathway to enhance motor neuron outgrowth defects, then it can be postulated that they may work in the Wnt pathway. As a result they must be dependent on the presence of Wnt pathway components to exhibit their functions. Alternatively, they are a class of proteins which function in an additional novel, unidentified pathway that is involved in regulation of guidance in motor neurons in the dorsal-ventral axis. In, $C$. elegans the known components of the Wnt pathway include five ligands, EGL 20, LIN44, MOM-2, CWN-1, CWN-2, four frizzled receptors, LIN-17/Frizzled, MOM-5, MIG1/Frizzled, CFZ-2 and one RYK/Derailed receptor tyrosine kinase, LIN-18 (Eisenmann, 2005). Since this mechanism involves a much greater amount of proteins, it is more difficult to establish whether ENU-3 and its paralogs function in this pathway but the fact that there are six members of the ENU-3 family and multiple Wnt cues and receptors might suggest that there could be a one to one correspondence between the Wnt pathway members and ENU-3. Numerous genetic constructions of mutants of ENU-3 and paralogs in mutant backgrounds of several components of the Wnt pathway, in addition to RNAi knockdowns should be able to attend to whether the ENU-3 protein family members are dependent on constituents of the Wnt pathway to control dorsal guidance of DA and DB motor neurons in C.elegans. Alternatively, Wntless prevents secretion of Wnt ligands so the knockout or knockdown of Wntless may be sufficient to observe if ENU-3 family members are in the same pathway as the Wnt pathway. The establishment of the expression patterns of the ENU-3 proteins could shed some light on this issue as the expression patterns of all of the Wnts are known. 


\subsection{ENU-3 and its paralogs may have roles in cytoskeletal rearrangement}

Genetic analysis data of unc-40(e1430);enu-3(tm4519);mec7::GFP mutants reveal additional defects to those affecting ventral guidance of AVM and PVM touch receptor neurons. It was noticeably seen that in these double mutants, there was a high frequency of animals with "zig-zaggged" kinks in their axons especially in the PLM, as opposed to a straight trajectory in wild-type animals. Furthermore, previous observations with enu3(tm4519);unc-5(e53);evIs82b mutants were consistent with this idea as some of the motor neuron axons appeared tangled or broken and did not reconnect at the point of the apparent break (Yee, 2011). Together these results suggest that ENU-3 may play a potential role in adhesion or integrity of the cytoskeleton by affecting the architecture of axons (Yee et al., 2014). DCC regulates adhesion of cells by directly interacting with UNC-6/Netrin in the substratum (Shekarabi et al., 2005). Although unc-40(e1430) mutants experience attachment defects of touch receptor neurons occasionally, the penetrance of this type of defect is mostly seen in mutants lacking both UNC-40 and ENU-3. This indicates that the two proteins may be co-operating to promote proper adhesion of axons to their substratum ultimately affecting the arrangement of the cytoskeleton in C. elegans (Yee et al., 2014).

The possible role in maintaining or establishing cytoskeleton arrangement is also supported and can be explained by the expression analyses of ENU-3, and two of its paralogs C38D4.1 and K01G5.3 in mammalian HeLa cells. Due to the feasibility of using a larger cell model in which different subcellular compartments can be easily identified, ENU-3 and one of the paralogs with a longer intracellular region, also predicted to contain nuclear localization signals and a trans-membrane domain, were first expressed in 
HeLa cells. Similarly, proteins involved in clathrin-mediated endocytosis, from a far more distantly related organism, the parasitic protist, Trypanosoma brucei, were analyzed through expression in mammalian cell models in order to identify if the proteins are related to those characterized in higher eukaryotes (Adung'a, Gadelha, \& Field, 2013). Thus expression analyses of proteins from distantly related organism in mammalian cell models are reliable and feasible techniques practiced among more recent research groups.

The nuclear membrane/ER localization of ENU-3 made it less likely to conclude that ENU-3 is a surface receptor working in parallel to UNC-40 to affect mechanosensory neuron migration. Rather, it supported the idea that the protein is working in the pathway of another surface receptor parallel to UNC-40 that can respond to sources of UNC-6 and affect migration of touch receptor neurons. Since the FLAG tag inserted at the $\mathrm{N}$-terminus was proven not to trap the protein in the ER and mask its true localization, it was concluded that a high concentration of the protein is truly observed along the nuclear membrane and ER. Partial co-localization expression of ENU-3 with an ER marker KDEL::GFP, further proved the authenticity of the expression pattern of ENU-3. Lastly, the finding that greatly supported the importance of the predicted transmembrane domain of ENU-3 strongly pointed towards the fact that the predicted transmembrane domain of ENU-3 is necessary for localization on the nuclear membrane and ER of HeLa cells thus providing an additional support for the authentic expression of ENU-3 on the nuclear membrane and ER of HeLa cells. Nuclear membrane proteins are known to interact directly with the actin cytoskeleton, thus these observations are encouraging since ENU-3 appears to have weak homology to various proteins involved in arrangement of the mammalian cytoskeletal system. The BFA treatment of cells 
containing ENU-3 without the trans-membrane domain, demonstrated that ENU-3 cannot be localized to the nuclear membrane without its trans-membrane domain. Additionally, it showed that ENU-3 is likely to be following the secretory pathway by undergoing cotranslational translocation dependently or independently of the Sec-61 complex into the ER and packaging into vesicular structures destined for secretion out of the cell if the TM domain is absent. C38D4.1 which had a similar cellular localization to ENU-3 is also predicted to behave in the same manner. However future experiments will confirm this hypothesis.

In contrast, the K01G5.3 protein which is not predicted to contain a trans-membrane domain was identified as a plasma membrane associated intracellular protein from its expression pattern in HeLa cells. It can be concluded that K01G5.3 may have a more direct role than ENU-3 and C38D4.1 in modification of the cytoskeleton with a possible role in actin interaction and modulation. It is likely that they are involved in regulating actin formation and turnover similar to the functions of characterized membraneassociated proteins such as fascin, N-WASP and the Arp 2/3 complex (Dickinson \& Purich, 2007). This is indicative of the expression pattern of K01G5.3 which showed several thin, finger-like structures protruding from the cell similar to filopodia which consist of actin filaments cross-linked by actin-binding proteins (Hanein, Matsudaira, \& DeRosier, 1997).

Interactions between the nuclear envelope and the cytoskeleton are vital for cell functionality (Starr, \& Fridolfsson, 2010). Membrane proteins on the ONM can interact with the cytoskeleton while proteins on the INM interact with nuclear lamina. The ONM and INM interact via the perinuclear space. SUN membrane proteins (Sad 1/UNC-84) 
located on the INM and ONM KASH proteins (Klarsicht, ANC-1, and Syne/Nesprin homology) form a bridge known as the KASHSUN nuclear envelope bridge model which aims to connect the cytoskeleton system with the nucleoskeleton system (Starr, \& Fridolfsson, 2010).

The understanding of this model was accomplished with the initial characterization of UNC-84, an INM protein, and ANC-1, an ONM protein, in C. elegans where mutants with deficits in those proteins showed defects in nuclear migration of $\mathrm{P}$ cells leading to missing neurons and ultimately an uncoordinated phenotype (Horvitz \& Soulson, 1980). Missing neurons, especially the PVM were also often seen with enu-3(tm4519);unc40(e1430) mutants, suggesting a similar role attributable to ENU-3. In addition, ENU-3 and C38D4.1 expressed with a distinct signal on the nuclear envelope similar to the pattern observed when UNC-84 and ANC-1 are expressed (Lee et al., 2002) thus strengthening the case that ENU-3 and C38D4.1 may interact with the already identified proteins, or at the very least they may share similar roles in generating the KASHSUN bridge required for downstream interaction with the cytoskeleton.

Furthermore, ENU-3 has 76 amino acids after the trans-membrane domain while C38D4.1 has comparatively more residues due to the longer intra-cellular region, up to the C-terminus. Thus, since all conserved KASH proteins contain a small 35-residue or less tail in the perinuclear space (Starr, \& Fridolfsson, 2010), this makes ENU-3 and C38D4.1 less likely to be ONM proteins, and more likely to be INM proteins that may facilitate interaction with nuclear lamina directly underneath the INM in order to affect cytoskeletal architecture of the cell (Chen et al., 2006). In addition to possessing a transmembrane domain for insertion into the INM, INM proteins are also characterized by 
having short coiled-coil regions, dimers or trimmers of alpha helices coiled together, towards their C-terminal tail that is facing the perinuclear space, critical for regulation of gene expression and other biological functions (Crisp et al., 2006). Similarly, ENU-3 and C38D4.1 contain predicted coiled-coil regions towards their C-terminal tail. Additionally, KASH proteins require INM SUN proteins for proper localization to the ONM (Crisp et al., 2006) thus ENU-3 and C38D4.1 may play a direct or indirect role working with UNC-84 on the INM to permit insertion of the ONM resident proteins in the ONM, thus forming the KASHSUN nuclear envelope bridge necessary for interactions between the nucleoskeleton and cytoskeleton. ENU-3 and C38D4.1 could function similarly to already characterized INM proteins in recruiting ONM proteins from the ER and facilitating their insertion into the ONM thereby permitting the nucleus to attach to the actin cytoskeleton of the cell, vital for cell viability.

\subsection{ENU-3 interacts with ZEN-4 and GEI-4}

Yeast-two-hybrid screens have shown that ENU-3 physically interacts with two wellknown proteins, ZEN-4 and GEI-4 which affect early development of nematodes (Li et al., 2004). ZEN-4 (zygotic epidermal enclosure defective) is present at the 2-cell stage of early development of the worm, and is necessary for polar body extrusion following meiotic divisions, for termination of cytokinesis after mitosis and for generation of spindle microtubules (Raich et al., 1998). Although ZEN-4 is characterized as a kinesinlike protein (Reich et al., 1998) which can travel along microtubules requiring ATP (Siddiqui et al., 2002) and is known to play a role in meiotic and mitotic divisions, axonal transport has also been demonstrated to be performed by ZEN-4 and kinesis proteins in general (Etchberger et al., 2007). Etchberger and colleagues (2007) have identified that 
kinesins are capable of additionally transporting neurotransmitters and receptors from the core to the surface of the cell.

A certain region of ENU-3 has been found to be similar to inhibitors of ATPase pumps, using BLAST analysis (Yee, 2011). Since, kinesins are ATPases (Siddiqui et al., 2002), it is possible that this domain permits the interaction between ZEN-4 and ENU-3. In this way, ZEN-4 could transport ENU-3 and other molecules bound to it towards the cell body for recycling which may be essential during the development of the nervous system. ZEN-4 may transport UNC-40 in addition to ENU-3, recycle the components and ultimately allow UNC-5 signalling to increase resulting in axon outgrowth in a wild-type manner.

GEI-4 (GEX interacting protein) is a protein which directly interacts with GEX-2 (Gut on Exterior), and is known to be involved in vulval morphogenesis as well as fertility and viability of embryos (Tsuboi et al., 2002). GEX-2 is known to function through a mechanism downstream of several axon guidance signalling pathways (Shakir et al., 2008), namely the WAVE pathway resulting in filopodia extensions of the developing growth cone (Shakir et al, 2008). Based on recent results, ENU-3 may play a role upstream of GEX-2, and the ENU-3 interaction with GEI-4, which in turn interacts with GEX-2, may reveal a novel unidentified role for ENU-3 similar to that of GEX-2. In support of this, enu-3 mutants did reveal partial embryonic lethality and males were typically infertile. Interestingly, the interacting complex between GEX-2 and GEI-4 was observed to be essential during embryogenesis as it was able to regulate formation of intermediate filaments during cytoskeletal rearrangement (Tsuboi et al., 2008). 


\subsection{Future experiments}

Even though there has been a considerable amount of progress achieved in understanding the roles of the ENU-3 protein family members, there remain several additional experiments to be performed in order to gain further insights into their contribution in the development of the nervous system in C. elegans.

\subsubsection{Localization experiments}

If ENU-3 does in fact play a role in affecting the localization of UNC-40 or UNC-5 on the cell surface of growth cones thereby altering the orientation of developing axons, then this can be observed by first obtaining an integrated line of translational function constructs of UNC-40 and UNC-5 which are readily available within the nematode community. These animals can then be crossed into enu-3(tm4519) mutants and observed for altered localization of UNC-40::GFP or UNC-5::GFP under an epifluorescence microscope. If these mutants also possess axon migration defects in the affected cells where a mislocalization of these receptors took place, then undoubtedly, a part of ENU3's role is to direct proper localization of these receptors which then allow for correct migration of developing axons.

\subsubsection{ENU-3 family members' expression patterns in C. elegans}

The expression patterns of the paralogs of ENU-3 through microinjection of transcriptional and translational reporters are imperative for advancing the current understanding of these paralogs. The promoter region and the gene of interest should be fused with GFP at the C-terminal through PCR, as previously done for ENU-3. The fusion constructs containing genomic wild-type copies of the paralogs and GFP should be micro-injected with a co-injection marker into wild-type animals. If the paralogs of ENU- 
3 do indeed play a role in axon outgrowth of motor neurons, their expression patterns should reveal at least partial concentration in the cell bodies of DA and DB motor neurons along the $\mathrm{VC}$.

\subsubsection{Determining the directionality of ENU-3 in dorsal-ventral guidance}

Genetic data has revealed that ENU-3 is clearly involved in directing axon guidance of the AVM and PVM touch receptor neurons; however its mechanism is still unclear. In order to prove that ENU-3 functions in the ventral-ward direction similar to UNC-40, which is what is believed based on the genetic knock-out results of the unc40(e1430);enu-3(tm4519) double mutant, it is imperative that ENU-3 is expressed in the mechanosensory neurons. This can be accomplished by microinjecting unc-40(e1430) animals with a genomic copy of ENU-3 fused with GFP, however its own promoter region must be substituted with the mec-7 promoter which permits expression of the translated protein in the mechanosensory neurons. If ENU-3 does in fact work in the ventral ward direction, then an overexpression of ENU-3 in unc-40 mutants, should supress the guidance defects as ENU-3 can over-compensate for the lack of UNC-40 and can direct developing axons towards the ventral cord as it may be chemo-attracted towards the sources of UNC-6. Alternatively, ENU-3 works in the dorsal direction and thus an enhancement of defects will be observed as ENU-3 may be chemo-repulsed by UNC-6 along the VC in the absence of UNC-40. The latter is less likely as genetic knock-down and knock-out data indicate that ENU-3 works parallel to UNC-40 supposedly in the same direction in aiding axons to migrate ventrally. 


\subsubsection{Determining if ENU-3 and its paralogs function in the Wnt pathway}

Recently, Levy Struumpf and Culotti (2014) have demonstrated that the Wnt pathway is also involved in guidance of neurons along the dorsal-ventral cord. Consequently, ENU-3 and its paralogs may function in this pathway to control dorsal migration of motor neurons, since genetic analysis has proven that they are not dependent on the constituents of the UNC-6/Netrin signalling pathway to exhibit their function. RNAi and genetic construction of mutants in this case will be more challenging as the Wnt pathway is regulated by a far more complicated mechanism and there are many more components to this pathway. Nevertheless genetic mutants between enu-3 and its paralogs as well as members of the Wnt pathway should not lead to an enhancement of defects if they do in fact function in the Wnt pathway. RNAi knockdown may provide a faster alternative when looking at the outgrowth defects of the DA and DB motor neurons; however the mutations' full effect will not be generated unless genetic mutants are constructed.

\subsubsection{Testing K01G5.3's ability to modify actin dynamics}

Since the expression pattern of K01G5.3 in mammalian cells was that of an intracellular membrane-associated protein with finger-like protrusions similar to filopodia, it would be interesting to observe if K01G5.3 does in fact modify actin dynamics and thus regulate overall cytoskeleton arrangement of the cell. In order to determine if this is the case, cells transfected with FLAG::K01G5.3 should be co-stained with an actin antibody in order to visualize the actin arrangement in comparison to a wild-type untransfected cell. If a distinct actin arrangement is seen in the cells transfected with K01G5.3, it can be concluded that this paralog is at least involved in actin 
modification, although it does not prove that K01G5.3 is directly affecting the rearrangement.

\subsubsection{Yeast-Two-Hybrid Screen}

Based on the results from the expression analyses of ENU-3 and two of its paralogs in HeLa cells, it would be interesting to observe if there are interactions between these proteins and a variety of cytoskeletal and actin-interacting proteins, such as UNC-78 (homologous to actin-interacting protein 1 in humans). In addition to those, I expect that ENU-3 and its four paralogs which contain a trans-membrane domain, to have possible interactions with INM and ONM proteins as well as lmn-1 (homologous to lamin in humans). Finally, an interaction between ENU-3 and its paralogs and members of the Wnt pathway will be observed if they truly function in the Wnt pathway with a newly identified role in the dorsal-ventral guidance of developing neurons. Newly identified interaction will provide further insight into the mechanism by which ENU-3 and its paralogs can affect guidance of developing neurons in C. elegans. 


\section{Chapter 5. Conclusions}

The development of the nervous system is an intricate process which is established in a stereo-typical fashion as interactions between gradient molecules and their respective receptors on the growth cones of developing neurons promote correct trajectories towards the target locations. The UNC-6/Netrin pathway is the mechanism by which several identified proteins function in order to control dorsal-ventral guidance of developing neurons, including the motor neurons and mechanosensory neurons examined in this thesis. ENU-3 is a novel trans-membrane protein which appears to have a role in directing guidance of AVM and PVM mechanosensory neurons, as well as affecting outgrowth of DA and DB motor neurons in a pathway independent of UNC-6/Netrin. The paralogs of ENU-3 which have a high similarity in protein structure amongst each other, are also involved in DA and DB motor neuron outgrowth of developing neurons. Expression analyses in HeLa cells have demonstrated additional potential roles for ENU3 and its paralogs in direct or indirect cytoskeletal rearrangement of cells. This is promising as neuronal architecture defects were also observed in unc-40(e1430);enu3(tm4519) mutants as well as weak homologies of ENU-3 with proteins involved in cytoskeleton modification were found.

All hypotheses have been proven in this work. It has been demonstrated that ENU3 functions downstream of UNC-6 to affect guidance of mechanosensory neurons. Additionally, all members of the ENU-3 protein family work redundantly and are involved in axon outgrowth of motor neurons. Lastly, it was proven that ENU-3 is a trans-membrane protein localizing to the nuclear membrane, while the paralog without the predicted trans-membrane domain localized to a different compartment in HeLa cells, 
namely intracellularly and plasma membrane associated. Future work will investigate the exact biochemical roles of these novel proteins in the nervous system development of C.elegans. 


\section{Chapter 6. Appendices}

In this section, I provide the primer sequences and PCR program used for the seamless cloning technique in the construction of FLAG::ENU-3, FLAG::ENU-3::GFP, ENU3::GFP, F::ENU-3::GFP without the trans-membrane domain, FLAG::C38D4.1 and FLAG::K01G5.3. In addition I will provide the primer sequence PCR details for the construction of the mec7p::ENU-3::GFP fusion construct.

\subsection{Generation of ENU-3, C38D4.1 and K01G5.3 epitope tagged constructs}

The cDNA ORF of each paralog was amplified from the Thermo Scientific cDNA ORF clone collection as well as amplification of the pcDNA3 mammalian expression vector done using primers that were partly homologous to the insert of interest and partly homologous to the pcDNA3 vector sequence at the site of insertion (Table 6.1). The amplicons were later transformed into competent cells where the plasmids would recombine through homologous recombination. The same PCR mixture of components and the same gradient PCR protocol were used to generate all constructs shown in Table 6.2 and 6.3 . 


\begin{tabular}{|c|c|}
\hline Primer Name & Sequence \\
\hline pcDNA 3 with enu-3 Insert $L$ & $\begin{array}{l}\text { GCT-CGG-ATC-CAC-TAG-TAA-CAT-GCG- } \\
\text { TAT-TTT-GCG-TAT-TTT-G }\end{array}$ \\
\hline pcDNA 3 with enu-3 Vector $L$ & $\begin{array}{l}\text { CAA-AAT-ACG-CAA-AAT-ACG-CAT- } \\
\text { GTT-ACT-AGT-GGA-TCC-GAG-C }\end{array}$ \\
\hline pcDNA 3 with enu-3 Vector $R$ & $\begin{array}{l}\text { GAC-AAG-CCA-CGC-GAT-TAG-AAG- } \\
\text { GTG-CCA-CTC-CC }\end{array}$ \\
\hline pcDNA 3 with enu-3 Insert $R$ & $\begin{array}{l}\text { GGG-AGT-GGC-ACC-TTC-TAA-TCG-CGT- } \\
\text { GGC-TTG-TC }\end{array}$ \\
\hline FLAG ENU-3 Left & $\begin{array}{l}\text { GGT-GGT-TGT-TGG-TAA-TAC-ATG-AAC- } \\
\text { TAT-TAA-TCC-GAA-G }\end{array}$ \\
\hline FLAG ENU-3 Right & $\begin{array}{l}\text { A-CCA-ACA-ACC-ACC-GAT-TAC-AAG- } \\
\text { GAT-GAC-GAC-GAT-AAG-ACT-ACT- } \\
\text { ACG-ACA-CCA-AAT-GTG }\end{array}$ \\
\hline $\begin{array}{l}\text { pcDNA3 with or without FLAG, and } \\
\text { GFP Insert L }\end{array}$ & $\begin{array}{l}\text { GAC-AAG-CCA-CGC-GAC-GGA-CTG- } \\
\text { AGA-TCT-AGA-TCC-CAA-ATG-GTG- } \\
\text { AGC-AAG }\end{array}$ \\
\hline $\begin{array}{l}\text { pcDNA3 with or without FLAG, and } \\
\text { GFP Vector L }\end{array}$ & $\begin{array}{l}\text { CTT-GCT-CAC-CAT-TTG-GGA-TCT- } \\
\text { AGA-TCT-CAG-TCC-GTC-GCG-TGG- } \\
\text { CTT-GTC }\end{array}$ \\
\hline $\begin{array}{l}\text { pcDNA3 with or without FLAG, and } \\
\text { GFP Vector } R\end{array}$ & $\begin{array}{l}\text { ACC-GGA-TCT-AGA-TAA-GAA-GGT- } \\
\text { GCC-ACT-CCC }\end{array}$ \\
\hline $\begin{array}{l}\text { pcDNA3 with or without FLAG, and } \\
\text { GFP Insert } R\end{array}$ & $\begin{array}{l}\text { GGG-AGT-GGC-ACC-TTC-TTA-TCT- } \\
\text { AGA-TCC-GGT }\end{array}$ \\
\hline
\end{tabular}




\begin{tabular}{|c|c|}
\hline pcDNA3 with C38D4.1 Insert L & $\begin{array}{l}\text { GAG-CTC-GGA-TCC-ACT-AGT-AAC- } \\
\text { ATG-CGT-ATT-TTG- CG }\end{array}$ \\
\hline pcDNA3 with C38D4.1 Vector $L$ & $\begin{array}{l}\text { CGC-AAA-ATA-CGC-ATG-TTA-CTA- } \\
\text { GTG-GAT-CCG-AGC-TC }\end{array}$ \\
\hline pcDNA3 with C38D4.1 Vector $R$ & $\begin{array}{l}\text { CCA-CGC-GAT-TGA-GAA-GGT-GCC- } \\
\text { ACT-CCC }\end{array}$ \\
\hline pcDNA3 with C38D4.1 Insert R & $\begin{array}{l}\text { GGG-AGT-GGC-ACC-TTC-TCA-ATC- } \\
\text { GCG-TGG }\end{array}$ \\
\hline FLAG C38D4.1 Right & $\begin{array}{l}\text { GGG-AGG-TCT-ATA-TAA-GAT-TAT- } \\
\text { AAA-GAT-GAT-GAT-GAC-AAA-GCA- } \\
\text { GAG-CTC-TCT-GGC }\end{array}$ \\
\hline FLAG C38D4.1 Left & $\begin{array}{l}\text { TAA-CTA-GAG-AAC-CCA-CTG-CTT- } \\
\text { ACT-GGC-TTA-TCG }\end{array}$ \\
\hline pcDNA3 with K01G5.3 Insert L & $\begin{array}{l}\text { GAG-CTC-GGA-TCT-ACT-AGT-AAC- } \\
\text { ATG-TGT-ATT-TGG-CGT }\end{array}$ \\
\hline pcDNA3 with K01G5.3 Vector L & $\begin{array}{l}\text { ACG-CCA-AAT-ACA-CAT-GTT-ACT- } \\
\text { AGT-AGA-TCC-GAG-CTC }\end{array}$ \\
\hline pcDNA3 with K01G5.3 Vector $R$ & $\begin{array}{l}\text { GAC-AAG-CCA-CAC-GAT-TGA-GAA- } \\
\text { GGT-GCC-ACT-CCC }\end{array}$ \\
\hline pcDNA3 with K01G5.3 Insert $R$ & $\begin{array}{l}\text { GGG-AGT-GGC-ACC-TTC-TCA-ATC- } \\
\text { GTG-TGG-CTT-GTC }\end{array}$ \\
\hline
\end{tabular}




\begin{tabular}{ll}
\hline FLAG K01G5.3 Right & GAG-ATC-AAG-ACT-CCC-GAT-TAT- \\
& AAA-GAT-GAT-GAT-GAC-AAA-AAT- \\
& GGA-AAC-GTC-ACA-AAA-GT \\
\hline FLAG K01G5.3 Left & GGG-AGT-CTT-GAT-CTC-TTC-TAC- \\
& CAC-ATA-CGG-ATT-CGC \\
\hline Deletion of ENU-3 TM domain Right & TTT-CAC-AAG-GAC-ACA-CCG-AAA- \\
& GAG-GAT-CAG-AAG-GCG \\
\hline Deletion of ENU-3 TM domain Left & CGC-CTT-CTG-ATC-TTC-TTT-CGG- \\
& TGT-GTC-CTT-GTG-AAA \\
\hline
\end{tabular}

Table 6.1: List of primers used to generate epitope tagged versions of ENU-3, C38D4.1, K01G5.3, and to delete the trans-membrane (TM) domain of ENU-3. 


\begin{tabular}{ll}
\hline Component & Volume \\
\hline $\mathrm{H}_{\mathbf{2}} \mathbf{O}$ & $80 \mathrm{uL}$ \\
$\mathbf{1 0 x}$ PFU Buffer & $10 \mathrm{uL}$ \\
DMSO & $5 \mathrm{uL}$ \\
\hline $\mathbf{1 0 M m}$ dNTP & $2 \mathrm{uL}$ \\
\hline PFU AD & $2 \mathrm{uL}$ \\
\hline Primers (use INS-L/INS-R or VECT-L/VECT-R) & $2 \mathrm{uL}$ of each primer \\
\hline DNA (use insert or vector) & $2 \mathrm{uL}$ \\
\hline
\end{tabular}

Table 6.2: Components of PCR mixture to amplify pcDNA3 and the three paralogs *split the above in 4 tubes (26.25 uL each for gradient PCR)

\begin{tabular}{llll}
\hline Step & Temperature & Time & Cycles \\
\hline Initial Denaturation & $95^{\circ} \mathrm{C}$ & $2 \mathrm{~min}$ & 1 \\
Denaturation & $95^{\circ} \mathrm{C}$ & & \\
Annealing & $40-64^{\circ} \mathrm{C}$ gradient & $1 \mathrm{~min}$ & 18 \\
Elongation & $68^{\circ} \mathrm{C}$ & $12 \mathrm{~min}$ & \\
& & & - \\
\hline
\end{tabular}

Table 6.3: PCR settings used to amplify pcDNA3 and the three paralogs 


\subsection{Generation of the $\Delta T M F:: E N U-3:: G F P$}

The construct F::ENU-3::GFP previously made was used as the template to remove its trans-membrane domain through site-directed mutagenesis in which the forward primer was homologous to the ENU-3 sequence up to the predicted trans-membrane domain at the $\mathrm{N}$ terminus and the $\mathrm{C}$ terminus of the primer was homologous to the sequence of ENU-3 immediately after the trans-membrane domain (Table 6.1). The reverse complement became the reverse primer. Similar PCR mixtures and gradient PCR protocol were used to generate this construct shown in Table 6.2 and 6.3.

\subsection{Generation of the mec7p::ENU-3::GFP construct for microinjection}

The mec-7 promoter was amplified from p1525 plasmid driving UNC-5 provided by Dr. Culotti Lab at Mt. Sinai Hospital using mec-7 forward and mec-7 Translational Fusion reverse primers. $3 \mathrm{~kb}$ of the promoter region and the gene region of ENU-3 $(1 \mathrm{~kb})$ was amplified from the H04D03.1 fosmid in addition to amplification of GFP from the pD95.75 plasmid. ENU-3 was then fused to GFP using primers and PCR protocol listed below. Finally, the promoter of ENU-3 was substituted with the mec7 promoter using the mec-7 translational fusion forward primer and GFP nested primer listed below. 


\begin{tabular}{ll}
\hline Primer Name & Sequence \\
\hline Outside H04D03.1 & CGT-GTA-ACT-CCT-TTC-ATC-TTG-GCA-ACC-ATA-GCT- \\
\hline Nested H04D03.1 & CTG-TTG-AAG-GAG-ATC-CTC-TTT \\
\hline Translational Fusion & AGT-CGA-CCT-GCA-GGC-ATG-CAA-GCT-ATC-GCG- \\
& TGG-CTT-GTC-CAC-AGT \\
\hline GFP Nested & GGA-AAC-AGT-TAT-GTT-TGG-TAT-A \\
mec-7 Forward & TCT-AGA-AAT-GTA- AAC-CTG-TCA- TTT \\
\hline mec-7 Translational Fusion & CA-CTT-TTT-TCG-CAA-GCC-TCG-CAC-GTT-TTT-TGC- \\
reverse & TTG-AAA-TTT-GGA-CCC G \\
\hline mec-7 Translational Fusion & C-GGG-TCC-AAA-TTT-CAA-GCA-AAA-AAC-GTG-CGA- \\
\hline
\end{tabular}

Table 6.4: List of primers used to generate the mec7p::enu-3::gfp construct

\begin{tabular}{ll}
\hline Component & Volume \\
\hline dNTP (500 uM each, mixed) & $2.5 \mathrm{uL}$ \\
\hline Upstream primer (300 $\mathbf{~ M )}$ & $1 \mathrm{uL}$ \\
\hline Downstream primer (300 $\mathbf{~ M )}$ & $1 \mathrm{uL}$ \\
\hline $\mathbf{1 0 x}$ PCR Buffer (System 2) & $5 \mathrm{uL}$ \\
\hline Template DNA & $1 \mathrm{uL}(80 \mathrm{ng})$ \\
\hline Expand Long Template Enzyme Mix & $0.75 \mathrm{uL}$ \\
\hline ddH $_{2} \mathbf{O}$ & $38.75 \mathrm{uL}$ \\
\hline
\end{tabular}

Table 6.5: PCR components to amplify the promoter and gene of enu-3, GFP and the mec7 promoter 


\begin{tabular}{llll}
\hline Step & Temperature & Time & Cycles \\
\hline Initial Denaturation & $94^{\circ} \mathrm{C}$ & $2 \mathrm{~min}$ & 1 \\
Denaturation & $94^{\circ} \mathrm{C}$ & $10 \mathrm{~s}$ & \\
Annealing & $55^{\circ} \mathrm{C}$ & $30 \mathrm{~s}$ & \\
Elongation & $68^{\circ} \mathrm{C}$ & 4 min & \\
Denaturation & $94^{\circ} \mathrm{C}$ & $15 \mathrm{~s}$ & \\
Annealing & $55^{\circ} \mathrm{C}$ & & \\
Elongation & $68^{\circ} \mathrm{C}$ & $30 \mathrm{~s}$ & \\
& & & \\
\hline Final Elongation & $68^{\circ} \mathrm{C}$ & 4 min $+20 \mathrm{~s}$ cycles elongation for & \\
\hline Cooling & $4{ }^{\circ} \mathrm{C}$ & each successive cycle & \\
\hline
\end{tabular}

Table 6.6: PCR settings to amplify GFP, the promoter and gene of enu-3, and the $m e c 7$ promoter

\begin{tabular}{ll}
\hline Component & Volume \\
\hline dNTP TaKaRa Mixture (2.5 $\mathbf{~ m M ~ e a c h ) ~}$ & $4 \mathrm{uL}$ \\
\hline Upstream Primer (300 $\mathbf{n M})$ & $1 \mathrm{uL}$ \\
\hline Downstream Primer (300 nM) & $1 \mathrm{uL}$ \\
\hline 10x Ex Taq Buffer & $5 \mathrm{uL}$ \\
\hline Promoter PCR product & $1 \mathrm{uL}(80 \mathrm{ng})$ \\
\hline GFP PCR product & $1 \mathrm{ul}(80 \mathrm{ng})$ \\
\hline TaKaRa Ex Taq (5units/uL) & $0.25 \mathrm{uL}$ \\
\hline ddH & \\
\hline
\end{tabular}

Table 6.7: PCR components for the fusion mixture of enu-3::gfp and mec7::enu-3::gfp 


\begin{tabular}{llll}
\hline Step & Temperature & Time & Cycles \\
\hline Initial Denaturation & $98^{\circ} \mathrm{C}$ & $2 \mathrm{~min}$ & 1 \\
Denaturation & $94^{\circ} \mathrm{C}$ & $10 \mathrm{~s}$ & 30 \\
Annealing & $55^{\circ} \mathrm{C}$ & $30 \mathrm{~s}$ & \\
Elongation & $72^{\circ} \mathrm{C}$ & 6 min & 1 \\
Final Elongation & $72^{\circ} \mathrm{C}$ & & - \\
Cooling & $4{ }^{\circ} \mathrm{C}$ & 10 min & \\
\hline
\end{tabular}

Table 6.8: PCR settings used to generate enu-3::gfp and the mec7::enu-3::gfp fusion constructs. 


\section{Chapter 7. References}

Ackerman, S. L., and Knowles, B. B. 1998. Cloning and mapping of the UNC5C gene to human chromosome 4q21-q23. Genomics 52 (2), 205-8.

Ackley, B. D., Crew, J. R., Elamaa, H., Pihlajaniemi, T., Kuo, C. J., Kramer, J. M. 2001. The Nc1/Endostatin domain of C.elegans Tyep Xviii collagen affects cell migration and axon guidance. J Cell Biol 152 (8), 1218-32.

Adler, C. E., Fetter, R. D., and Bargmann, C. I. 2006. UNC-6/Netrin induces neuronal asymmetry and defines the site of axon formation. Nat Neurosci. 9 (4), 511-8.

Adung'a, V. O., Gadelha, C., and Field, M. C. 2013. Proteomic Analysis of Clathrin Interactions in Trypanosomes reveal dynamic evolution of endocytosis. Traffic 14 (4), 440-457.

Antonescu, C. 2013. Seamless cloning workshop presentation. Ryerson University.

Antonescu, C.N., Danuser, G., Schmid, S.L. 2010. Phosphatidic acid plays a regulatory role in clathrin-mediated endocytosis. Mol Biol Cell 21, 2944-52.

Asikainen, S., Vartiainen, S., Lakso, M., Nass, R., Wong, G. 2005. Selective sensitivity of caenorhabditis elegans neurons to RNA interference. Neuroreport 16(18), 199599.

Berkowitz, L. A., Knight, A. L., Caldwell, C. A., Caldwell, K. A. 2008. Generation of stable transgenic C.elegans using microinjection. J Vis Exp 18, 833.

Bibel, M., and Barde, Y. A. 2000. Neurotrophins: Key regulators of cell fate and cell shape in the vertebrate nervous system. Genes \& Dev. 14, 2919-37.

Brenner, S. 1974. The genetics of Caenorhabditis elegans. Genetics 77, 71-94.

C. elegans sequencing consortium. 1998. Genome sequence of the nematode C. elegans: A platform for investigating biology. Science 282(5396), 2012-2018. 
Chalfie, M., Sulston, J. E., White, J. G., Southgate, E., Thomson, J. N., Brenner, S. 1985.

The neural circuit for touch sensitivity in caenorhabditis elegans. J. Neurosci. 5 (4), 956-64.

Chan, S. S., Zheng, H., Su, M., Wilk, R., Killeen, M. T., Hedgecock, E. M., Culotti, J. G.1996. UNC-40, a C. elegans homolog of DCC (deleted in colorectal cancer), is required in motile cells responding to UNC-6 netrin cues. Cell 87(2), 187-95.

Chang, C., Yu, T., Bagmann, C. I., and Tessier-Lavigne, M. 2004. Inhibition of netrinmediated axon attraction by a receptor protein tyrosine phosphatase. Science (New York, N. Y.) 305 (5680), 103-106.

Chen, B.I., Huber, M., Guan, T., Bubeck, A., Gerace, L.2006. Nuclear envelope transmembrane proteins (NETs) that are up-regulated during myogenesis. BMC Cell Biology 7 (38), doi:10.1186/1471-2121-7-38.

Chen, X., and Burgoyne, R. D. 2012. Identification of common genetic modifiers of neurodegenerative diseases from an integrative analysis of diverse genetic screens in model organisms. BMC Genomics 13(1).

Colamarino, S. A., and Tessier-Lavigne, M. 1995. The axonal chemoattractant netrin-1 is also a chemorepellent for trochlear motor axons. Cell 81 (4), 621-9.

Colavita, A., Krishna, S., Zheng, H., Padgett, R.W., Culotti, J.G. 1998. Pioneer axon guidance by UNC-129, a C. elegans TGF-beta. Science 28, 706-709.

Connerly, P. L. 2010. How Do Proteins Move Through the Golgi Apparatus? Nature Education 3(9), 60. 
Cooper, G. 2000. The nuclear envelope and traffic between the nucleus and cytoplasm.

The Cell: A Molecular Approach, $2^{\text {nd }}$ ed. Sunderland (MA): Sinauer Associates.

Costaguta, C. and Payne, G. S. 2009. Overview of Protein Trafficking Mechanisms. Trafficking Inside Cells: Pathways, Mechanisms, and Regulation. Los Angeles (CA): Landes Bioscience and Springer Science+Business Media.

Crisp, M., Liu, Q., Roux, K., Rattner, JB., Shanahan, C., et al. 2006. Coupling of the nucleus and cytoplasm: role of the LINC complex. J. Cell Biol. 172:41-53.

Desai, C., and Horvitz, H. R. 1989. Caenorhabditis elegans mutants defective in the functioning of the motor neurons responsible for egg laying. Genetics 121 (4), 703721.

Dickinson, R. B.and Purich, D. L. 2007. Nematode Sperm Motility: Nonpolar Filament Polymerization Mediated by End-Tracking Motors. Biophysical Journal 92 (2), $622-631$.

Dudanova, I., and Klein, R. 2013. Integration of guidance cues: parallel signalling and crosstalk. Trends Neurosci. 36 (5), 295-304.

Eisenmann, D.M. (2005) Wnt signaling. WormBook: 1-17 doi:10.1895/wormbook.1.7.1

Etchberger, J. F., Lorch, A., Sleumer, M. C., Zapf, R., Jones, S. J., Marra, M. A. et al. 2007. The molecular signature and cis-regulatory architecture of a C.elegans gustatory neuron. Genes \& Dev. 21(13), 1653-74.

Fraser, A. G., Kamath, R. S., Zipperlen, P., Martinez-Campos, M., Sohrmann, M., Ahringer, J. 2000. Functional genomics analysis of C.elegans chromosome I by systematic RNA interference. Nature 408 (6810), 325-330. 
Fujisawa, K., Wrana, J. L. and Culotti, J. G. 2007. The slit receptor EVA-1 coactivates a SAX-3/Robo mediated guidance signal in C.elegans. Science (New York, N. Y.) 317 (5846), 1934-1938.

Garibyan, L., and Avashia, N. 2013. Polymerase Chain Reaction. J of Investigative Dermatology 133 (6), doi:10.1038/jid.2013.1.

Gerace, L., Blum, A. and Blobel, G. 1978. Immunocytochemical localization of the major polypeptides of the nuclear pore complex-lamina fraction. Interphase and mitotic distribution. J. Cell Biol. 79, 546-566.

Goldberg, J. L. 2003. How does an axon grow?. Genes \& Dev. 17, 941-958.

Greenfield, J. A., and High, S. 1999. The Sec61 complex is located in both the ER and the ER-Golgi intermediate compartment. Journal of Cell Science 112, 147799.

Gruenbaum, Y., Margalit, A., Goldman, R.D., Shumaker, D.K., Wilson, K.L. 2005. The nuclear lamina comes of age. Nat Rev Mol Cell Biol 6, 21-31.

Hall, D.H., and Altun, Z. F. 2008. C.elegans Atlas. Cold Spring harbor laboratory press (Cold Spring Harbor, New York), 62-70.

Hall, D. H., Lints, R., and Altun, Z. 2005. Nematode neurons: Anatomy and anatomical methods in caenorhabditis elegans. Int. Rev. Neurobiol. 69, 1-35.

Hanein, D., Matsudaira, P., DeRosier, D.J. 1997. Evidence for a conformational change in actin induced by fimbrin (N375) binding. J. Cell Biol. 139 (2), 387-96.

Hao, J. C., Yu, T., Fujisawa K., Culotti, J. G., Gengyo-Ando, K., Mitani, S. et al. 2001. C.elegans slit acts in midline, dorsal-ventral, and anterior-posterior guidance via the SAZ-3/Robo receptor. Neuron 32 (1), 25-38. 
Heber, S., Herms, J., Gajic, V., Hainfellner, J., Aguzzi, A., Rulicke, T., Muller, U. 2000. Mice with combined gene knock-outs reveal essential and partially redundant functions of amyloid precursor protein family members. J. Neurosci 20 (21), 795163.

Hedgecock, E. M., Culotti, J. G., Hall, D. H. 1990. The unc-5, unc-6, and unc-40 genes guide circumferential migrations of pioneer axons and mesodermal cells on the epidermis in C. elegans. Neuron 4 (1), 61-85.

Hillier, L. W., Coulson, A., Murray, J. I., Zhirong, B.Soulston, J. E., Waterston, R. H. 2005. Genomics in C.elegans: So many genes, such a little worm. Genome Res 15, 1651-60.

Hipolito, Victoria. 2014. Using forward and reverse genetics in C.elegans to study motor neuron outgrowth defects. Undergraduate thesis, Ryerson University.

Hiramoto, M., Hiromi, Y., Giniger, E., and Hotta, Y. 2000. The Drosophila netrin receptor Frazzled guides axons by controlling netrin distribution. Nature 406 (6798), 886-9.

Hobert, O. 2002. PCR fusion-based approach to create reporter gene constructs for expression analysis in transgenic C.elegans. BioTechniques 32 (4), 728-730.

Hong, K., Hinck, L., Nishiyama, M., Poo, M. M., Tessier-Lavigne, M., Stein, E. 1999. A ligand-gated association between cytoplasmic domains of UNC-5 and DCC family receptors convert netrin-induced growth cone attraction to repulsion. Cell 97 (7), 927-41.

Hope, I. 1999. C. elegans: A practical Approach; Oxford University Press: Oxford. Horvitz, H.R., and Sulston, J.E. 1980. Isolation and genetic characterization of celllineage mutants of the nematode Caenorhabditis elegans. Genetics 96, 435-54. 
Ishii, N., Wadsworth, W. G., Stern, B. D., Culotti, J. G., Hedgecock, E. M. 1992. UNC-6, a laminin-related protein, guides cell and pioneer axon migrations in C. elegans. Neuron 9 (5), 873-881.

Itzkovitz, S., Baruch, L., Shapiro, E., and Segal, E. 1992. Geometric constraints on neuronal connectivity facilitate a concise synaptic adhesive code. P. Natl. Acad. Sci. USA. 105 (27), 9278-9283.

Kamath, R. S. 2003. Ahringer, J. Genome-wide RNAi screening in Caenorhabditis elegans. Methods 30 (4), 313-321.

Keino-Masu, K., Masu, M., Hinck, L., Leonardo, E.D., Chan, S. S., Culotti, J. G., Tessier-Lavigne, M. 1996. Deleted in Colorectal Cancer (DCC) encodes a netrin receptor. Cell 87 (2), 175-85.

Keleman, K. and Dickinson, B.J. 2001. Short- and long-range repulsion by the Drosophila UNC5 netrin receptor. Neuron 32, 605-17.

Kennedy, M. B. 1998. Signal transduction molecules at the glutamatergic postsynaptic membrane. Brain Res. Rev. 26 (2-3), 243-257.

Kennedy, T. E., Serafini, T., de la Torre, J. R., and Tessier-Lavigne, M. 1994. Netrins are diffusable chemotropic factors for commissural axons in the embryonic spinal cord. Cell 78 (3), 425-35.

Killeen, M. T., and Sybingco, S. S. 2008.Netrin, Slt and Wnt receptors allows axons to choose the axis of migration. Dev Bio. 323, 143-151. 
Killeen, M., Tong, J., Krizus, A., Steven, R., Scott, I., Pawson, T.,et al. 2002. UNC-5 function requires phosphorylation of cytoplasmic tyrosine 482, but its UNC-40 independent functions also require a region between the $\mathrm{ZU}-5$ and death domains. Dev Bio 251 (2), 348-366.

Kolodkin, A. L., and Tessier-Lavigne, M. 2011. Mechanisms and molecules of neuronal wiring: a primer. Cold Spring Harb Perspect Biol 3 (6).

Lai, W., Sun, K., Correia, J. P., and Kennedy, T. E. 2011. Netrins: versatile extracellular cues with diverse functions. Development 138 (11), 2153-69.

Lee, J., Li, W., and Guan, K. L. 2005. SRC-1 mediates UNC-5 signalling in C.elegans. Molecular and Cellular Biology 25 (15), 6485-95.

Lee, K.K., Starr, D.A., Cohen, M., Liu, J., Han, M., et al. 2002. Lamin-dependent localization of UNC-84, a protein required for nuclear migration in C. elegans. Mol. Biol. Cell 13, 892-901.

Leonardo, E. D., Hinck, L., Masu, M., Keino-Masu, K., Ackerman, S. L., and TessierLavigne, M. 1997. Vertebrate homologues of C. elegans UNC-5 are candidate netrin receptors. Nature 386 (6627), 833-8.

Leung-Hagesteijn, C., Spence, A. M., Stern, B. D., Zhou, Y., Su, M. W., Hedgecock, E. M. and Culotti, J. G. 1992. UNC-5, a transmembrane protein with immunoglobulin and thrombospondin type 1 domains guides cell and pioneer axon mirations in C. elegans. Cell 71 (2), 289-99.

Levy-Strumpf, N., and Culotti, J. G. 2014. Netrins and Wnts function redundantly to regulate antero-posterior and dorso-ventral guidance in C.elegans. PLoS Genet. 10 (6), e1004381. 
Li, W., Lee, J., Vikis, H. G., Lee, S. H., Liu, G., Aurandt, J., et al. 2004. Activation of FAK and src receptor-proximal events required for netrin signaling. Nature Neuroscience 7 (11), 1213-21.

Lim, Y.S., and Wadsworth, W.G. 2002. Identification of domains of netrin UNC-6 that mediate attractive and repulsive guidance and responses from cells and growth cones. J Neurosci 22, 7080-7087.

Lindsay, R. M., Thoenen, H., and Barde, Y. A. 1985. Placode and neural crest-derived sensory neurons are responsive at early developmental stages to brain-derived neurotrophic factor. Dev. Biol. 112, 319-328.

Liu, G., Beggs, H., Jurgensen, C., Park, H. T., Tang, H., Gorski, J., et al. 2004. Netrin requires focal adhesion kinase and src family kinases for axon outgrowth and attraction. Nature Neuroscience 7 (11), 1222-32.

Lundquist, E.A., and Norris, A. D. 2011. UNC-6/netrin and its receptors UNC-5 and UNC-40/DCC modulate growth cone protrusion in vivo in C. elegans. Development 138, 4433-4442.

MacNeil, L. T., Hardy, W. R., Pawson, T., Wrana, J. L., and Culotti, J. G. 2009. UNC129 regulates the balance between UNC-40 dependent and independent UNC-5 signalling pathways. Nature Neuroscience 12 (2), 150-155.

Mahmood, T., and Yang, P. 2012. Western Blot: Technique, Theory, and Trouble Shooting. N AM J Med Sci. 4 (9), 429-43.

Maskery, S., and Shinbrot, T. 2005. Deterministic and stochastic elements of axonal guidance. Annual Review of Biomedical Engineering 7, 187-221.

McIntire, S.L., Jorgensen, E., Kaplan, J. and Horvitz, H.R. 1993. The GABAergic nervous system of Caenorhabditis elegans. Nature 364, 337-341. 
Misumi, Y., Misumi, Y., Miki, K., Takatsuki, A., Tamura, G., and Ikehara, Y. 1986. Novel blockage by brefeldin A of intracellular transport of secretory proteins in cultured rat hepatocytes. J Biol Chem 261, 11398-403.

Mitchell, K. J., Doyle, J. L., Serafini, T., Kennedy, T. E., Tessier-Lavigne, M., Goodman, C.S., and Dickson, B. J. 1996. Genetic analysis of netrin genes in Drosophila: Netrins guide CNS commissural axons and peripheral motor axons. Neuron 17 (2), 203-15.

Munson, M. and Hughson, F.M. 2002. Conformational regulation of SNARE assembly and disassembly in vivo. J. Biol. Chem. 277, 9375-9381.

Murgatroyd, C., and Spengler, D. 2010. Histone tales: Echoes from the past, prospects for the future. Genome Biol. 11 (2).

Nasu-Nishimura, Y.,Hurtado, D., Braud, S., Tnag, T.T., Isaac, J., and Roche, K.V. 2006. Identification of an endoplasmic reticulum retention motif in an intracellular loop in the Kainate Receptor subunit KA2. J Neurosci 26 (26), 7014-21.

Palani, D., Pekala, D., Baginskas, A., Szkudlarek, H., Raastad, M. 2012. Action potentials recorded from bundles of very thin, gray matter axons in rat cerebellar slices using a grease-gap method. J. Neurosci. Meth., 208 (2), 119-127.

Quinn, L. 2005. Seamless cloning and gene fusion. TRENDS in Biotechnology 23 (4).

Raich, W. B., Moran, A. N., Rothman, J. H. and Hardin, J. 1998. Cytokinesis and midzone microtubule organization in C.elegans require the kinesin-like protein ZEN-4. Molec Biol of the Cell 9(8), 2037-49.

SAS Institute. Statistical Analysis System (SAS) [computer software]. SAS Institute. 2013. Retrieved from: https://vapps.ryerson.ca/Citrix/XenApp. 
Serafini, T., Kennedy, T.E., Galko, M.J., Mirzayan, C. J., Thomas M.,Tessier-Lavigne, M. 1994. The netrins define a family of axon outgrowth-promoting proteins homologous to C. elegans UNC-6. Cell 78 (3), 409-24.

Shakir, M. A., Jiang, K., Struckhoff, E. C., Demarco, R. S., Patel, F. B., Soto, M. C., et al. 2008. The Arp 2/3 activators WAVE and WASP have distinct genetic interactions with rac GTPases in C. elegans axon guidance. Genetics 179 (4), 195771.

Shaw, G., and Bray, D. 1977. Movement and extension of isolated growth cones. Exp Cell Research 104 (1), 55-62.

Shekarabi, M., Moore, S.W., Tritsch, N.X., Morris, S.J., Bouchard, J.F., Kennedy, T.E. 2005. Deleted in colorectal cancer binding netrin-1 mediates cell substrate adhesion and recruits Cdc42, Rac1, Pak1, and N-WASP into an intracellular signaling complex that promotes growth cone expansion. J Neurosci $\mathbf{2 5}, 3132$ 3141.

Siddiqui, S. S. 2002. Metazoan motor models: Kinesis superfamily in C.elegans. Traffic (Copenhagen, Denmark) 3 (1), 20-28.

Starr, D. A., and Fridolfsson, H. N. 2010. Interactions between nuclei and the cytoskeleton are mediated by SUN-KASH Nuclear-envelope bridges. Annu Rev Cell Dev Biol. 28, 421-444.

Stuurman, N., Heins, S., and Aebi, U. 1998. Nuclear lamins: their structure, assembly, and interactions. J Struct Biol 122, 42-66.

Sulston, J.E., Albertson, D.G., and Thomson, J.N. 1980. The Caenorhabditis elegans male: postembryonic development of nongonadal structures. Dev. Biol. 78, $542-576$. 
Timmons, L., Court, D. L., and Fire, A. 2001. Ingestion of bacterially expressed dsRNAs can produce specific and potent genetic interference in caenorhabditis elegans. Gene 263(1-2), 103-112.

Tojima, T. 2012. Intracellular signalling and membrane trafficking control bidirectional growth cone guidance. Neurosci Res. 73 (4), 269-74.

Tong, J., Killeen, M., Steven, R., Binns, K. L., Culotti, J., and Pawson, T. 2001. Netrin stimulates tyrosine phosphorylation of the UNC-5 family of netrin receptors and induces Shp2 binding to the RCM cytodomain. The J of Biol Chem 276 (44), 40917-25.

Tsuboi, D., Qadota, H., Kasuya, K., Amano, M., and Kaibuchi, K. 2002. Isolation of the interacting molecules with GEX-3 by a novel functional screening. Biochemical and Biophysical Research Communications 292 (3), 697-701.

Vitriol, E. A., and Zheng, J. Q. 2012. Growth cone travel in space and time: the cellular ensemble of cytoskeleton, adhesion, and membrane. Neuron 73 (6), 1068-81.

Wadsworth, W. G. 2002. Moving around ina worm: Netrin UNC-6 and circumferencial axon guidance in C.elegans. Trends in Neuroscience 25 (8), 423-429.

Wang, X., Roy, P. J., Holland, S. J., Zhang, L. W., Culotti, J. G., and Pawson, T. 1999. Multiple ephrins control cell organization in C.elegans using kinase-dependent and -independent functions of the VAB-1 eph receptor. Molecular Cell 4 (6), 903-913.

White, J. G., Southgate, E., Thomson, J. N., and Brenner, S. 1986. The structure of the nervous system of the nematode C. elegans. Philos. T. ROY. Soc. B. 314 (1165), 1340. 
Wilson, K. L., and Berk, J. M. 2010. The nuclear envelope at a glance. J of Cell Science 123, 1973-78.

Wong, J.T., Yu, W. T., and O’Connor, T. P. 1997. Transmembrane grasshopper semaphoring I promotes axon outgrowth in vivo. Development (Cambridge, England) 124 (18), 3597-3607.

WormBase. 2014. WormBase Version: WS240 C. elegans domain [database]. National Human Genome Research Institute. Retrieved from: www.wormbase.org

Worman, H.J. and Gundersen, G.G. 2006. Here come the SUNs: a nucleocytoskeletal missing link. Trends Cell Biol 16, 67-69.

Xu, Z., Li, H., and Wadsworth, W. G. 2009. The roles of multiple UNC-40(DCC) receptor-mediated signals in determining neuronal asymmetry induced by the UNC-6 (netrin) ligand. Genetics 183 (3), 941-9.

Yam, P. T., and Charron, F. 2013. Signalling mechanisms of non-conventional axon guidance cues: the Shh, BMP, and Wnt morphogens. Curr Opin Neurobiol. 23 (6), 965-73.

Ye, Q., and Worman, H.J. 1996. Interaction between an integral protein of the nuclear envelope inner membrane and human chromodomain proteins homologous to Drosophila HP1. J Biol Chem 271, 14653-56.

Yee, C.S. 2011. ENU-3 is a novel axon outgrowth and guidance protein in C.elegans. Masters Thesis, Ryerson University.

Yee, C. S., Sybingco, S. S., Serdetchania, V., Kholkina, G., Bueno de Mesquita, M., Naqvi, Z., Killeen, M. T. 2011. ENU-3 is a novel motor axon outgrowth and guidance protein in C. elegans. Dev. Biol. 352 (2), 243-253. 
Yee, C.S., Florica, R., Fillingham, J., Killeen, M.T. 2014. ENU-3 functions in an UNC6/Netrin dependent pathway parallel to UNC-40/DCC/Frazzled for outgrowth and guidance of the touch receptor neurons in C.elegans. Developmental Dynamics 243, $459-467$.

Zallen, J. A., Yi, B. A., and Bargmann, C. I. 1998. The conserved immunoglobulin superfamily member SAX-3/Robo directs multiple aspects of axon guidance in C.elegans. Cell 92 (2), 217-27. 